\title{
The role of ciliates, heterotrophic dinoflagellates and copepods in structuring spring plankton communities at Helgoland Roads, North Sea
}

\author{
Martin G. J. Löder · Cédric Meunier • \\ Karen H. Wiltshire • Maarten Boersma • \\ Nicole Aberle
}

Received: 25 August 2010/Accepted: 4 March 2011

(C) Springer-Verlag 2011

\begin{abstract}
Mesocosm experiments coupled with dilution grazing experiments were carried out during the phytoplankton spring bloom 2009. The interactions between phytoplankton, microzooplankton and copepods were investigated using natural plankton communities obtained from Helgoland Roads $\left(54^{\circ} 11.3^{\prime} \mathrm{N} ; 7^{\circ} 54.0^{\prime} \mathrm{E}\right)$, North Sea. In the absence of mesozooplankton grazers, the microzooplankton rapidly responded to different prey availabilities; this was most pronounced for ciliates such as strombidiids and strobilids. The occurrence of ciliates was strongly dependent on specific prey and abrupt losses in their relative importance with the disappearance of their prey were observed. Thecate and athecate dinoflagellates had a broader food spectrum and slower reaction times compared with ciliates. In general, high microzooplankton potential grazing impacts with an average consumption of $120 \%$ of the phytoplankton production $\left(P_{p}\right)$ were measured. Thus, the decline in phytoplankton biomass could be mainly attributed to an intense grazing by microzooplankton. Copepods were less important phytoplankton grazers consuming on average only $47 \%$ of $P_{p}$. Microzooplankton in turn contributed a substantial part to the copepods' diets especially with decreasing quality of phytoplankton food due to nutrient limitation over the course of the bloom. Copepod grazing rates exceeded microzooplankton growth, suggesting their strong top-down control potential on microzooplankton in the field.
\end{abstract}

Communicated by U. Sommer.

M. G. J. Löder $(\varangle)$ · C. Meunier · K. H. Wiltshire .

M. Boersma · N. Aberle

Biologische Anstalt Helgoland, Alfred Wegener

Institute for Polar and Marine Research,

POB 180, 27483 Helgoland, Germany

e-mail: Martin.Loeder@awi.de
Selective grazing by microzooplankton was an important factor for stabilising a bloom of less-preferred diatom species in our mesocosms with specific species (Thalassiosira spp., Rhizosolenia spp. and Chaetoceros spp.) dominating the bloom. This study demonstrates the importance of microzooplankton grazers for structuring and controlling phytoplankton spring blooms in temperate waters and the important role of copepods as top-down regulators of microzooplankton.

\section{Introduction}

Since Azam et al. (1983) introduced the term "microbial loop", microzooplankton has received ever increasing attention as an important structural and functional group in planktonic ecosystems. Indeed, microzooplankton is one of the major functional groups in microbial food webs (Landry and Calbet 2004) and links the smaller planktonic unicellular organisms with higher metazoan trophic levels (Sherr et al. 1986; Gifford and Dagg 1988; Stoecker and Capuzzo 1990). It contributes substantially to mesozooplankton diets (Kleppel 1993). Furthermore, microzooplankton facilitates the rapid recycling of nutrients back to primary producers (Calbet and Saiz 2005; Irigoien et al. 2005). Microzooplankton is both prey and competitor for mesozooplankton. A literature synthesis by Landry and Calbet (2004) revealed that microzooplankton grazing can account for $60-75 \%$ of the mortality of phytoplankton production across a spectrum of open ocean and coastal systems and therefore may exert a stronger grazing pressure on phytoplankton than copepods (Sherr and Sherr 2007). During bloom events, unicellular microzooplankton can respond quickly to increasing phytoplankton availability (Johansson et al. 2004; Aberle et al. 2007) with cell division rates in the same range as those of its prey. The 
combination of its faster metabolism and higher production compared to mesozooplankton (Fenchel and Finlay 1983; Müller and Geller 1993; Montagnes and Lessard 1999) with no egg and larval stages allows microzooplankton a rapid and direct response to prey availability when compared to mesozooplankton competitors.

The success of any phytoplankton species depends on having a gross growth rate that exceeds the losses due to physical (e.g. advection, mixing) and biological factors (notably predation). In combination with abilities that allow competitive advantage against other phytoplankters (e.g. higher maximum growth rate, higher substrate affinities, higher photosynthetic efficiency) even small differences in any of these factors can have profound effects explaining competitive advantage in growth capacity (Flynn 2008).

Irigoien et al. (2005) proposed that phytoplankton blooms occur when an external perturbation (e.g. more light and nutrients) breaks down the equilibrium in the formerly stable system by promoting better growth conditions and opens a "loophole" in the microbial loop. The improved growth conditions at the onset of a bloom allow phytoplankton species to escape predation pressure by microzooplankton. At this time, stocks and total grazing rates of herbivorous protists are not sufficiently high enough to suppress bloom formation (Sherr and Sherr 2009). In fact, total exclusion from grazing is not required for a particular phytoplankton species for the formation of a bloom; only a positive difference between growth rate and grazing rate resulting in positive net growth, while phytoplankton competitors may remain controlled by zooplankton grazing.

According to Irigoien et al. (2005), these loopholes for phytoplankton blooms are widened by the combined effects of (1) mesozooplankton predation on microzooplankton and (2) inter-microzooplankton predatory activity and thus grazing reduction on phytoplankton (trophic cascade effect) and (3) defence mechanisms (e.g. size, colonyformation, toxicity, spines) and (4) bad nutritional status of the algae and thus consequently predator avoidance (deselection effect). The latter presupposes that predators can actively choose their prey and show preference or avoidance tactics for specific prey items.

Foraging strategies are fundamental to trophic ecological considerations, e.g., trophic cascade effects, and some copepod species, for example, are known to choose specific food actively related to taxonomic differences of the prey (Gentsch et al. 2009), prey size (Paffenhöfer 1988), nutrient composition of the prey (Cowles et al. 1988) and related to their own life stages (Mauchline 1998). There is also evidence for selective feeding by microzooplankton species (Verity 1991) regarding particle size or taxonomic differences of the prey (Fenchel 1980; Stoecker et al. 1981; Jonsson 1986; Hansen 1992; Hamels et al. 2004). However, our knowledge on microzooplankton food selectivity is scarce in contrast to knowledge on selective feeding of mesozooplankton. This is attributed to methodological difficulties associated with ecological work on planktonic microzooplankton. Most microzooplankton species are fragile, not easy to handle and difficult to culture (Gifford 1985). Hence, laboratory investigations on feeding behaviour have focused on easily cultivable species.

Given the diverse feeding modes even within the microzooplankton community (Tillmann 2004), food preference and selectivity are also likely to be highly diverse in this group of grazers. As there is still only little knowledge on the cumulative influence of those factors on phytoplankton bloom assemblages (Brussaard et al. 1995; Aberle et al. 2007), investigations on microzooplankton grazing under conditions as close to nature as possible are imperative. In this context, special emphasis should be placed on interspecific interactions in the plankton as well as the total effect of changes in selectivity of the present grazers.

Our investigations focused on North Sea spring plankton communities at Helgoland Roads. Although this station has been sampled for plankton since 1962, the microzooplankton has only recently been investigated more thoroughly (Löder 2010). With their year-round occurrence and high biomass in the plankton at Helgoland Roads (Löder et al. 2011), heterotrophic protists can be expected to play an important role as phytoplankton grazers. In this study, we hypothesise that: (1) microzooplankton with its various feeding modes can have a considerable impact on phytoplankton spring blooms, which is potentially higher than the impact of copepods, (2) selective grazing by microzooplankton leads to blooms of less-preferred phytoplankton species, (3) microzooplankton succession in spring can be directly linked to the availability of different prey and (4) microzooplankton contributes substantially to copepods' diets, and copepods are therefore an important top-down control factor for herbivorous protists.

\section{Materials and methods}

We conducted a mesocosm experiment and simulated a natural spring bloom using in situ plankton communities from Helgoland Roads. Top-down control (e.g. copepod grazing) on microzooplankton can be severe (Sherr and Sherr 2009), especially in a restricted mesocosm environment, and can cause strong trophic cascade effects (Sommer et al. 2003; Sommer and Sommer 2006; Zöllner et al. 2009), thus hindering exclusive investigations on the direct effects of microzooplankton grazing on phytoplankton. We therefore excluded mesozooplankton grazers from the incubations. By relaxing microzooplankton from the grazing pressure of mesozooplankton, we could explicitly 
examine the role of microzooplankton grazing on phytoplankton communities during the bloom. Grazing experiments for detailed investigations on microzooplankton grazing and selectivity were conducted at four defined points of the phytoplankton spring bloom: pre-bloom (exponential growth phase $=$ experiment 1 ), bloom peak (biomass maximum $=$ experiment 2 ), early post-bloom (1 week after biomass maximum $=$ experiment 3 ) and later post-bloom (2 weeks after biomass maximum $=$ experiment 4 ). The role of copepods in structuring the spring phyto- and microzooplankton community was also examined via measuring copepod grazing and selectivity during these distinct bloom phases.

\section{Sampling site}

Helgoland is located in the German Bight (Southern North Sea). It is subject to both coastal influences from the shallow Wadden Sea as well as marine influences from the open North Sea. Since 1962, water samples are taken workdaily as part of a long-term monitoring for plankton and nutrients at the "Kabeltonne" site at Helgoland Roads $\left(54^{\circ} 11.3^{\prime} \mathrm{N} ; 7^{\circ} 54.0^{\prime} \mathrm{E}\right)$ (Wiltshire et al. 2008). Water for the mesocosm experiment was taken at this site.

\section{Set-up}

The aim of the mesocosm experiment was to follow a typical spring plankton succession under near-natural conditions. The mesocosm experiment took place from mid-March until mid-April 2009 in a constant temperature room with a starting temperature of $4.2^{\circ} \mathrm{C}$ and a quick rise towards the end temperature of $\sim 6.8^{\circ} \mathrm{C}$ within a few days. The tanks were heated by the increasing room temperature. Start and end temperatures were close to in situ conditions $\left(4.2^{\circ} \mathrm{C} / 6.7^{\circ} \mathrm{C}\right)$. In contrast to the field where temperature increased almost linearly, the rise in temperature in the mesocosms was somewhat faster and $6.5^{\circ} \mathrm{C}$ were reached on day four of the experiment.

Three cylindrical mesocosms with a volume of $750 \mathrm{~L}$ each were filled with natural seawater from Helgoland Roads. Water was first repeatedly scooped from the water surface using an open 850-L container suspended from the crane of the research vessel Uthörn, and three 1,000-L containers were subsequently filled by hose via gravity feed. In order to remove mesozooplankton but to allow for the passage of chain-forming diatoms and microzooplankton, the water was screened over the feed using a $200-\mu \mathrm{m}$ gauze bag connected to the end of the hose, which floated in the container during filling. Back on land, this water was transferred to the mesocosms again via gravity feed. Pumps were not used to ensure the survival of the whole plankton community and particularly delicate organisms (Löder et al. 2010). The even distribution of the water from each container to the three mesocosms was ensured by an interconnected triple-split hose distributor mounted on the main hose. Thus, after the filling of each mesocosm, they contained identical over-wintering/spring populations of bacteria, phytoplankton and zooplankton smaller than $200 \mu \mathrm{m}$ (microzooplankton).

The mesocosms were gently stirred by a propeller (107.5 rpm, $15 \mathrm{~min}$ on, then $15 \mathrm{~min}$ off). The propeller produced currents similar to that found in the field at Helgoland Roads (mean $0.6 \mathrm{~m} \mathrm{~s}^{-1}, \max 1.2 \mathrm{~m} \mathrm{~s}^{-1}$ ), ensured the continuous mixing of the water column and avoided sedimentation of the plankton. Light was provided by computer-controlled light units (Profilux II, GHL Groß Hard- and Software Logistics, Kaiserslautern, Germany) operated via an external control computer (Programme 'Prometheus', GHL, modified version 'Copacabana'). The light units were equipped with two different fluorescent tubes to obtain full light spectra ('Solar Tropic' and 'Solar Nature', JBL, Neuhofen, Germany), enabling the simulation of triangular light curves (see Sommer et al. 2007 for details). The light cycle and intensity was adjusted daily by the computer to account for changes in the photoperiod (same light cycle as in the field) during the experimental run according to the geographical position of Helgoland following the model by Brock (1981). A light intensity of $60 \%$ of surface irradiance without cloud cover was chosen, simulating the intensity of light at $1.50 \mathrm{~m}$ water depth with a light attenuation coefficient of 0.34 (5 m Secchi depth) under in situ conditions. Calculation of the light intensity was done via equations given by Tyler (1968) and Poole and Atkins (1929).

\section{Stocking with natural inocula}

During early seasonal succession, many planktonic organisms hatch from cysts, resting eggs or other resting stages at different times. Inoculating the mesocosms with one start community only would preclude the effects of later excystment or hatching. Therefore, to render similar successive patterns of the plankton in the mesocosms as in the field possible, we introduced a small inoculum of natural seawater from Helgoland Roads on a weekly basis. Five litres of $200 \mu \mathrm{m}$ screened seawater was added to each mesocosm. An additional $15 \mathrm{~L}$ of filtered seawater $(0.2 \mu \mathrm{m})$ were added to the mesocosms to compensate for evaporation and water removal due to the sampling for monitoring and experiments.

Sampling the mesocosms

Daily measurements of temperature, $\mathrm{pH}$ and in vivo fluorescence (chlorophyll a) (Algae Analyser, BBE 
Moldaenke, Kiel, Germany) were conducted between 08:00 and 09:00 h. In addition to the daily measurements, three litres of each mesocosm were sampled and analysed every Monday, Wednesday and Friday and as well as on days with grazing experiments.

Silicate, phosphate and DIN (nitrite, nitrate and ammonia) were determined colorimetrically after filtration of at least $0.3 \mathrm{~L}$ of sample through $0.45-\mu \mathrm{m}$ nylon filters (Falcon) following the methods of Grasshoff et al. (1999).

For the determination of phytoplankton species composition during the course of the experiment, $100 \mathrm{~mL}$ of the sample were subsampled into amber bottles and immediately fixed with neutral Lugol's iodine solution (final conc. 0.5\%) (Throndsen 1978). For the determination of the microzooplankton, $250 \mathrm{~mL}$ were fixed with acid Lugol's iodine solution immediately (final conc. 2\%) (Throndsen 1978). Samples were stored cool and dark. For phytoplankton species determination $25 \mathrm{~mL}$ and for microzooplankton $50 \mathrm{~mL}$ of the sample were settled in sedimentation chambers (HYDRO-BIOS) for $24 \mathrm{~h}$, and one replicate per mesocosm (total $\mathrm{n}=3$ ) was counted under a Zeiss Axiovert 135 inverted microscope using the Utermöhl method (Utermöhl 1958).

Counting procedure for the samples of the grazing experiments was principally the same as for the succession in the mesocosms but with higher replication, i.e., the whole set of incubation bottles (see detailed description below) was sampled and one subsample of each sample was counted.

Phytoplankton (diatoms, phytoflagellates except dinoflagellates) and microzooplankton (ciliates, dinoflagellates and others) were identified where possible to genus or species level or, otherwise, pooled into size-dependent groups or "morphotypes" (see taxa in the detailed tables of the "Appendix"). It is known that most chloroplast-bearing dinoflagellates are capable of mixotrophic nutrition via phagotrophy (Du Yoo et al. 2009). Therefore, all dinoflagellate species were considered potentially heterotrophic and were assigned to the microzooplankton. For microzooplankton, the whole surface of the sedimentation chamber was counted at 200-fold magnification to reduce counting biases against rare species. Phytoplankton was counted according to the standard method applied for the Helgoland Roads long-term phytoplankton data archive (Wiltshire and Dürselen 2004; Wiltshire et al. 2008). The whole surface of the chamber, or at least 100 cells or chains per abundant taxon, were counted when enumeration was "in tracks". The identification of phytoplankton and dinoflagellates was primarily based on Dodge (1982), Tomas (1996) and Hoppenrath et al. (2009). Ciliates were determined based on Kahl (1932), Carey (1992) and Montagnes (2003).

Phytoplankton and microzooplankton net growth rates in the mesocosms were calculated using the exponential growth model and the abundance values from the monitoring (see section "Growth and grazing calculations").

\section{Grazing experiments}

Dilution experiments on microzooplankton grazing (Landry 1993; Landry and Hassett 1982) and bottle incubations with the copepod Temora longicornis were carried out simultaneously at four different times (as described earlier) of the phytoplankton bloom (Fig. 1c).

\section{Microzooplankton grazing set-up}

A stock of water for the purpose of dilution was collected at the same time as the mesocosms were filled. Water was filtered at low pressure through a pre-washed $0.45+0.2$ $\mu \mathrm{m}$ sterile inline membrane filter capsule (Sartobran ${ }^{\circledR} 300$, $300 \mathrm{~cm}^{2}$ ) after pre-filtering with a combination of $3-\mu \mathrm{m}$ GFF $+0.2-\mu \mathrm{m}$ membrane filter. The sterile water was stored in the dark in the thermo-constant room together with the mesocosms. Particle freeness was checked via flow cytometry (FACS Calibur, Becton \& Dickinson) before each experiment started. Three exact dilutions of 10 , 25,50 and $100 \%$ of undiluted seawater from each mesocosm were prepared in carboys (Landry 1993). For the incubation, three $2.3-\mathrm{L}$ polycarbonate bottles were gently filled with water from each dilution carboy $(n=36: 3$ mesocosms $\times 4$ dilution levels $\times 3$ replicates). The Funnel-Transfer-Technique appropriate for ciliates (Löder et al. 2010) was used for filling purposes as these organisms are very sensitive to destruction by vigorous filling and mixing procedures (Landry 1993).

To prevent nutrient limitation biases in the microzooplankton grazing bottles, sterile filtered nutrient solutions (F/2 medium, Guillard and Ryther 1962) were added to the dilution series (Landry and Hassett 1982; Landry 1993) $\left(8 \times 10^{-4} \mathrm{mNO}_{3}, \quad 1.3 \times 10^{-5} \mathrm{mPO}_{4}\right.$ and $2.4 \times 10^{-5}$ $\mathrm{mSiO}_{2}$, experiment $1+2$ no $\mathrm{SiO}_{2}$ ). One bottle of undiluted water per mesocosm was incubated without the addition of nutrients to serve as control for the nutrient addition.

Samples for initial concentrations of microzooplankton and phytoplankton $(250 \mathrm{~mL}$, acid Lugol's iodine solution, final conc. $2 \%$ ) in the dilution experiments were obtained from the premix carboys of the dilution series in triplicate; after incubation for $24 \mathrm{~h}$, each incubation bottle was sampled. Microzooplankton and phytoplankton concentration in the samples was estimated as described earlier.

\section{Copepod grazing set-up}

The best method to the objective of this study for the quantification of feeding rates of mesozooplankton on both 

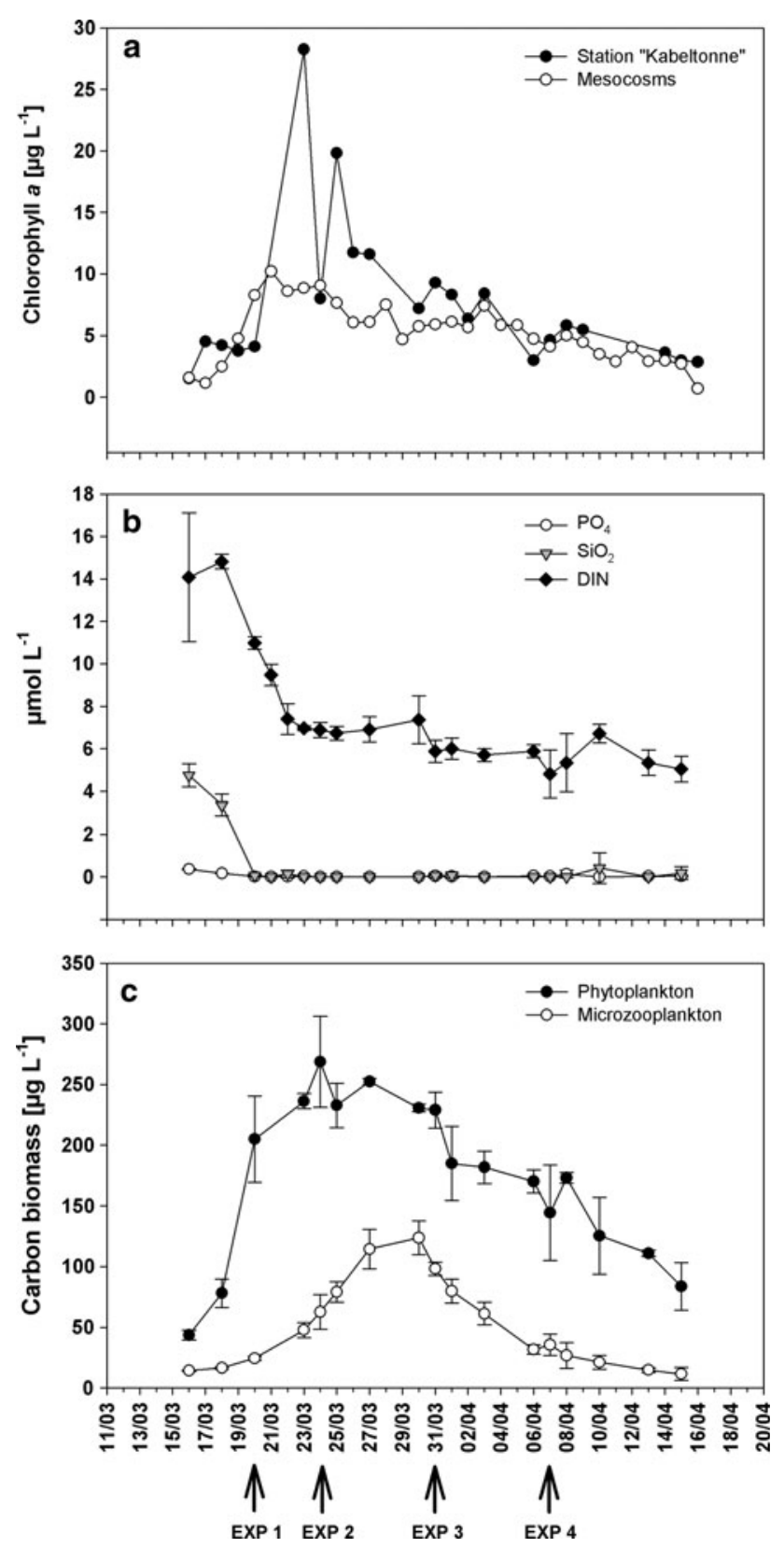

Fig. 1 Mean values of chlorophyll $a$ (in situ fluorescence) in the mesocosms as compared to the field station "Kabeltonne" (a), development of nutrients in the mesocosms (b) as well as the development of phytoplankton and microzooplankton biomass during the mesocosm experiment (c), dates at which the grazing experiments were performed are marked with arrows, error bars correspond to one standard deviation $(n=3)$

phytoplankton and non-pigmented microzooplankton was the analysis of particle removal in bottle incubations (Båmstedt et al. 2000). Because of interferences with microzooplankton grazing activity, especially when both micro- and mesozooplankton prey upon the same species, it is necessary to simultaneously estimate the microzooplankton grazing rates in separate dilution experiments
(Nejstgaard et al. 1997, 2001). Thus, for copepod grazing experiments three 2.3-L bottles per mesocosm (100\% undiluted water with added nutrients) were filled from carboys prepared along with the dilutions, and 25 female copepods of the species Temora longicornis were added to each bottle $\left(\sim 11\right.$ copepod $\left.\mathrm{L}^{-1}\right)(\mathrm{n}=9: 3$ mesocosms $\times 3$ replicates). This copepod concentration was at the upper limit of in situ densities in the period March-April (Greve et al. 2004).

The copepods were caught by vertical net hauls at Helgoland Roads and transferred to the laboratory immediately. Only actively swimming females of $T$. longicornis were sorted and acclimated to the mesocosm conditions for $24 \mathrm{~h}$ prior to the experiments.

Pre-condition of the copepods took place at the same temperature and light conditions as for the mesocosms in 3-L glass beakers containing mesocosm water. The $100 \%$ undiluted bottles in the dilution series served as a control for the T. longicornis grazing experiments. Sampling for plankton concentrations took place at the beginning of the $T$. longicornis grazing experiments and after $24 \mathrm{~h}$ as described for the microzooplankton grazing set-up. Plankton concentration in the samples was estimated as described earlier.

The whole set of incubation bottles (39 dilution series $+9 T$. longicornis $=48$ bottles) was incubated for $24 \mathrm{~h}$ on two plankton wheels $(0.8 \mathrm{rpm})$ under the same light and temperature conditions as the mesocosms.

Biovolume and carbon calculation

Biovolume of each plankton species was calculated from the measurement of cell dimensions using geometric formulae according to Hillebrand et al. (1999). The cell volume was converted into carbon (C) according to the equations given by Menden-Deuer and Lessard (2000) for diatoms $\left(\mathrm{pgC}\right.$ cell $\left.^{-1}=0.288 \times \mathrm{V}^{0.811}\right)$, dinoflagellates $\left(\mathrm{pgC} \quad\right.$ cell $\left.^{-1}=0.760 \times \mathrm{V}^{0.819}\right)$ and all other protist plankton except ciliates $\left(\mathrm{pgC}\right.$ cell $\left.^{-1}=0.216 \times \mathrm{V}^{0.939}\right)$, whereby $\mathrm{V}$ refers to cell volume in $\mu^{3}$. Ciliate carbon was calculated using a conversion factor of $0.19 \mathrm{pgC} \mu \mathrm{m}^{-3}$ (Putt and Stoecker 1989). Rotifer carbon was estimated according to McCauley (1984) and Park and Marshall (2000): After a calculation of the biovolume by means of geometric formulae, this biovolume was converted to wet weight assuming a specific gravity of 1 . Wet weight was then converted to dry weight by a factor of 0.1 and $50 \%$ of dry weight was assumed to be carbon. Carbon values for the copepod species $T$. longicornis were derived from measurements with an elemental analyser (EA 1110 CHNS-O, Thermo-Finnigan). The mean spring carbon content $\left(10 \mu \mathrm{g}\right.$ carbon female ${ }^{-1}$, March/April 2007, 
$\mathrm{n}=12$; Schoo, K. L. unpublished data) of this copepod was used in this study.

Growth and grazing calculation: microzooplankton

Growth rates of phytoplankton species and grazing rates of the microzooplankton community were calculated using linear regressions of apparent phytoplankton growth (calculated at a taxon level, for phytoplankton groups as well as for the total phytoplankton community) against the dilution factor (Landry and Hassett 1982; Landry 1993). Start values for the diluted samples were calculated from the $100 \%$ undiluted samples according to their dilution factor. The growth of phytoplankton $\left(\right.$ day $\left.^{-1}\right)$ was described by the exponential growth model in Eq. (1):

$C_{t_{24}}=C_{t_{0}} \times e^{(k-g) \times \Delta t}$

whereby $C_{t 0}$ is the concentration of phytoplankton biomass at the beginning of the experiment, $C_{t 24}$ after $24 \mathrm{~h}, k$ is the phytoplankton growth coefficient, $g$ is the microzooplankton grazing coefficient and $\Delta t$ is the incubation time in days.

Where in our experiments nonlinearity induced by saturated feeding of microzooplankton (Gallegos 1989) was seen, especially in experiments 1 and 4 where predator abundance was low, only the diluted samples $(10,25,50 \%)$ were used for regression analysis (Paterson et al. 2008). The obtained value of apparent phytoplankton growth was used to calculate the grazing coefficient at $100 \%$ undiluted seawater level. For comparisons between microzooplankton and mesozooplankton grazing, we normalised grazing parameters according to predator carbon concentration:

Daily carbon-specific grazing rates $g_{c}$, filtration rates $F_{c}$ and ingestion rates $I_{c}$ of the microzooplankton community were calculated for average (during the time interval $t_{0}-t_{24}$ ) prey carbon concentrations $\left[C_{\text {prey }}\right]$ after Frost (1972) with $g$ and $k$ obtained from the dilution experiments. $F_{c}$ and $I_{c}$ was adjusted for the growth of predators using mean predator carbon concentration $\left[C_{\text {predator }}\right]$ according to Heinbokel (1978) with Eqs. (2)-(5):

$F_{c}=g \times\left[C_{\text {predator }}\right]^{-1}$

$I_{c}=F_{c} \times\left[C_{\text {prey }}\right]$

$\left[C_{\text {prey }}\right]=\frac{C_{t_{0}} \times\left(e^{(k-g) \times \Delta t}-1\right)}{(k-g) \times \Delta t}$

$\left[C_{\text {predator }}\right]=\left(\frac{C_{\text {predator }, t_{24}}-C_{\text {predator }, t_{0}}}{\ln C_{\text {predator }, t_{24}}-\ln C_{\text {predator }, t_{0}}}\right)$

The instantaneous (natural) growth rate of phytoplankton $\mu_{0}$ was calculated by adding grazing mortality to values of apparent phytoplankton growth obtained from the incubation bottles without added nutrients (Landry 1993;
Caron 2000); negative values of $g$ were set to zero for calculation. Based on the coefficients obtained for $\mu_{O}$ and $g$ applied on the initial phytoplankton biomass $C_{t}$, the loss (\%) of phytoplankton standing crop per day $P_{i}$ and the loss (\%) of potential phytoplankton production $P_{p}$ of each species were calculated according to Eqs. (6) and (7) (Quinlan et al. 2009).

$P_{i}=\frac{C_{t_{0}} \times\left(e^{g}-1\right)}{C_{t_{0}}} \times 100$

$P p=\frac{C_{t_{0}} \times\left[\left(e^{\mu_{0}}-1\right)-\left(e^{\left(\mu_{0}-g\right)}-1\right)\right]}{C_{t_{0}} \times\left(e^{\mu_{0}}-1\right)} \times 100$

Copepod grazing: correcting for trophic cascade effects

The uncorrected grazing coefficient $g_{\mathrm{cop}, p}$ of $T$. longicornis was calculated for all prey types $p$ (taxon level, phytoplankton/microzooplankton groups, total phytoplankton/ microzooplankton community) after Frost (1972) at average prey concentrations, whereby the undiluted seawater incubation bottles of the dilution experiments served as control. As bottle incubations, containing several trophic levels (phytoplankton, microzooplankton and mesozooplankton), face the problem of insignificant, low or even statistically significant negative grazing rates (Nejstgaard et al. 1997, 2001), significance of grazing was tested after correction for trophic cascade effects.

The corrected copepod grazing coefficient ( $g_{\text {corr }, p}$, Eq. 8) was calculated following the general method of Nejstgaard (2001) by adding a correction factor $k_{p}$ for reduced microzooplankton grazing rates due to predation on micrograzers by $T$. longicornis to $g_{\mathrm{cop}, p}$ :

$g_{\text {corr }, p}=g_{\text {cop }, p}+k_{p}$

$k_{p}=g_{\text {micro }, p} \times\left(\frac{\left[C_{\text {predator }}\right]-\left[C_{\text {predator }}\right] *}{\left[C_{\text {predator }}\right]}\right)$

whereby $\left[C_{\text {predator }}\right]$ in Eq. (9) is the mean microzooplankton carbon concentration in the undiluted seawater from the dilution series and $\left[C_{\text {predator }}\right]^{*}$ is the mean microzooplankton carbon concentration in the $T$. longicornis bottles. Only significant microzooplankton grazing rates (linear regression analysis, $P<0.05$ ) were used for the correction, negative grazing rates were set to zero. We calculated rates of filtration $\left(F_{i}\right)$ and ingestion $\left(I_{i}\right)$ per individual of T. longicornis. To allow for comparisons with the microzooplankton, carbon-specific grazing $\left(g_{c}\right)$ and filtration rates $\left(F_{c}\right)$ and carbon-specific ingestion rates $\left(I_{c}\right)$ (phytoplankton and microzooplankton prey) of the added $T$. longicornis were calculated as described earlier for the microzooplankton. Contrary to the growth of phytoplankton microzooplankton growth was assumed not to be influenced by nutrient addition, and therefore, values of $k$ obtained for microzooplankton growth $k$ from the $100 \%$ undiluted 
seawater incubation bottles were taken to calculate $P_{i}$ and $P_{p}$ instead of $\mu_{0}$.

Sometimes negative $P_{i}$ and $P_{p}$ values were found in our grazing experiments. These resulted from either negative $g$ (for $P_{i}$ ) or $\mu_{0} / k$ (mortality in the control without grazer) (for $P_{p}$ ) and thus were set to zero. The same was done for positive $P_{p}$ values resulting from negative $g$ and $\mu_{0}$.

\section{Selectivity and electivity}

Prey selectivity $\alpha$ of the microzooplankton community and T. longicornis was calculated for each prey type (see above) according to Chesson $(1978,1983)$ (Eq. 10):

$\alpha_{i}=\frac{r_{i} / n_{i}}{\sum_{j=1}^{m} r_{j} / n_{j}}$

whereby $r_{i}$ is the frequency of prey $i$ in the diet and $n_{i}$ is the frequency of prey in the environment, divided by the sum of all relationships between the frequency of prey in the diet and in the environment. Negative T. longicornis ingestion rates were set to zero for the calculation of the frequency of prey in the diet according to Nejstgaard (2001). We chose Chesson's case 1 equation $\left(n_{i}\right.$ assumed to be constant) (Chesson 1983) because our values of ingestion and percentage of prey in the environment were obtained by averaged prey concentrations and phytoplankton initial stocks were high, so that a strong depletion of food was unlikely.

Values of $\alpha$ were used to calculate the electivity index $E^{*}$ according to Vanderploeg and Scavia (1979a, b) (Eq. 11).

$E^{*}=\frac{\alpha_{i}-\frac{1}{n}}{\alpha_{i}+\frac{1}{n}}$

( $n=$ total number of prey types).

Values of $E^{*}$ cover a range from -1 to $1 . E^{*}$ values of 0 indicate non-selective feeding, values $>1$ indicate preference, values $<1$ indicate discrimination against a prey type.

Data analysis

To monitor possible negative effects of our set-up technique on abundances, we statistically compared ( $t$ tests) microzooplankton communities at the start of the experiments in the mesocosms with the communities in the experimental bottles for differences. Insignificance was a requirement for applying the results of the experiments to the mesocosms. Regression analyses for the determination of $k$ and $g$ in the dilution experiments were conducted using "SigmaPlot 9.0" (SYSTAT Software); further statistical analyses were conducted using the software "Statistica 7.1" (StatSoft). Values of $g, k, F, I$ and $E^{*}$ obtained in the $T$. longicornis grazing experiments were tested against zero using two-tailed $t$ tests (Köhler et al. 1995). Significance levels of 0.05 were chosen in our analyses.

\section{Results}

Developments in the mesocosms

\section{Nutrients}

In the first 4 days of bloom development phosphate (start $0.36 \mu \mathrm{mol} \mathrm{L}{ }^{-1}$ ) and silicate (start $4.75 \mu \mathrm{mol} \mathrm{L}^{-1}$ ) decreased rapidly to values below detection limit $\left(<0.01 \mu \mathrm{mol} \mathrm{L}^{-1}\right)$ and no relaxation from nutrient limitation could be observed during the course of the experiment. Dissolved inorganic nitrogen (DIN) dropped from around $14 \mu \mathrm{mol} \mathrm{L}{ }^{-1}$ to around $7 \mu \mathrm{mol}$ $\mathrm{L}^{-1}$ after the first week and remained at a level between 5 and $7 \mu \mathrm{mol} \mathrm{L}{ }^{-1}$ during the rest of the experiment (Fig. 1b).

\section{General development of the spring bloom}

Starting with $44 \mu \mathrm{gC} \mathrm{L}{ }^{-1}$ phytoplankton biomass, similar to the bloom development in the field, the mesocosm spring bloom was initiated immediately and reached its maximum at $269 \mu \mathrm{gC} \mathrm{L} \mathrm{L}^{-1}$ (chlorophyll $a: 9 \mu \mathrm{g} \mathrm{L}^{-1}$, Fig. 1a) within the first 8 days of the experiment (24.03.09) (Fig. 1c). At this exponential phase, phytoplankton had a maximal net growth rate of 0.48 day $^{-1}$ (18.-20.03.09) and a mean net growth rate of $0.23 \mathrm{day}^{-1}$ (16.-24.03.09). During the following 3 weeks, the biomass decreased at a mean rate of $-0.05 \mathrm{day}^{-1}$ to a final value of $84 \mu \mathrm{gC} \mathrm{L}^{-1}$.

Microzooplankton, starting with $14 \mu \mathrm{gC} \mathrm{L}^{-1}$, followed the phytoplankton bloom with a delay of roughly one week and peaked on the 30.03.09 with $124 \mu \mathrm{gC} \mathrm{L}{ }^{-1}$ (Fig. 1c). Microzooplankton reached growth rates of up to 0.27 day $^{-1}$ (23.-24.03.09) but grew at a mean rate of 0.16 day $^{-1}$. Until the end of the experiment, microzooplankton biomass decreased at a mean rate of -0.15 day $^{-1}$ to $12 \mu \mathrm{gC} \mathrm{L}{ }^{-1}$, i.e. close to the starting value.

\section{Phytoplankton composition}

The spring bloom was dominated by diatoms and small flagellates (five size classes of 5-25 $\mu \mathrm{m}$ length, details in the "Appendix") (Fig. 2a, b). While flagellates contributed $34 \%$ to phytoplankton biomass at the start, they played only a minor role during the bloom phase. The bloom itself was principally build up by the same taxa as in the field (Löder et al., unpublished data) and was dominated by diatoms (96\%) of the genera Chaetoceros (C. danicus and other Chaetoceros spp. of different size classes), Thalassiosira (T. rotula and T. nordernskjoeldii) and Rhizosolenia ( $R$. stylisformis/hebetata group and $R$. pungens), each 


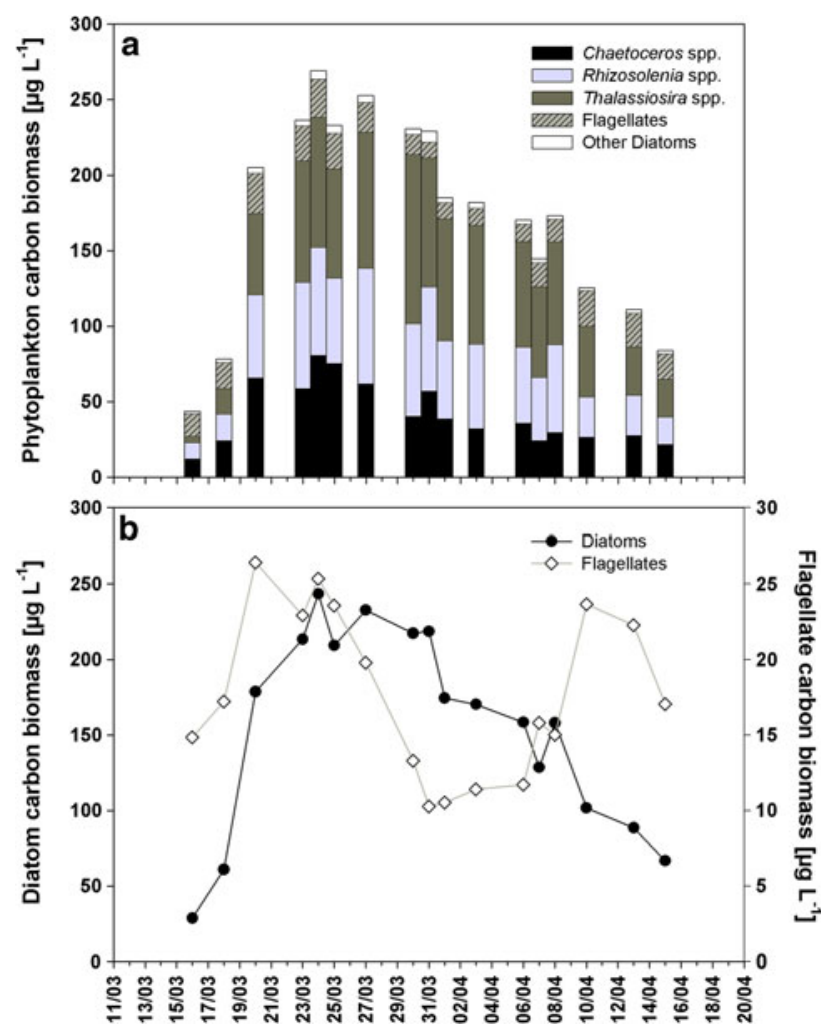

Fig. 2 Phytoplankton species succession (a) and development of diatom and flagellate carbon biomass (b) during the mesocosm experiment, mean values of the three mesocosms

genus contributing roughly one-third. In the later bloom phase, Thalassiosira became more dominant and contributed up to $49 \%$ to the phytoplankton carbon. The category 'other diatoms' (Pseudonitzschia spp., Navicula spp., Asterionellopsis glacialis and others) contributed only 1-4\% to the phytoplankton biomass. Flagellate biomass peaked 4 days earlier than the diatoms and showed a steeper decline. Along with decreasing diatom shares, it increased again contributing around $20 \%$ to the total phytoplankton carbon in the last week of the experiment.

\section{Microzooplankton composition}

Microzooplankton (Fig. 3) comprised four groups: dinoflagellates, ciliates, rotifers and thecate amoebae, whereby ciliates and dinoflagellates dominated during the first 3 weeks of the experiment. Due to their very low abundances, other metazoans like copepod nauplii or polychaete larvae were detected only sporadically and were neglected in our analyses.

\section{Ciliate community}

After a week of stability $\left(\sim 6 \mu \mathrm{gC} \mathrm{L}^{-1}\right)$, ciliate biomass increased at rates of around $0.36 \mathrm{day}^{-1}$ from 23.03.09

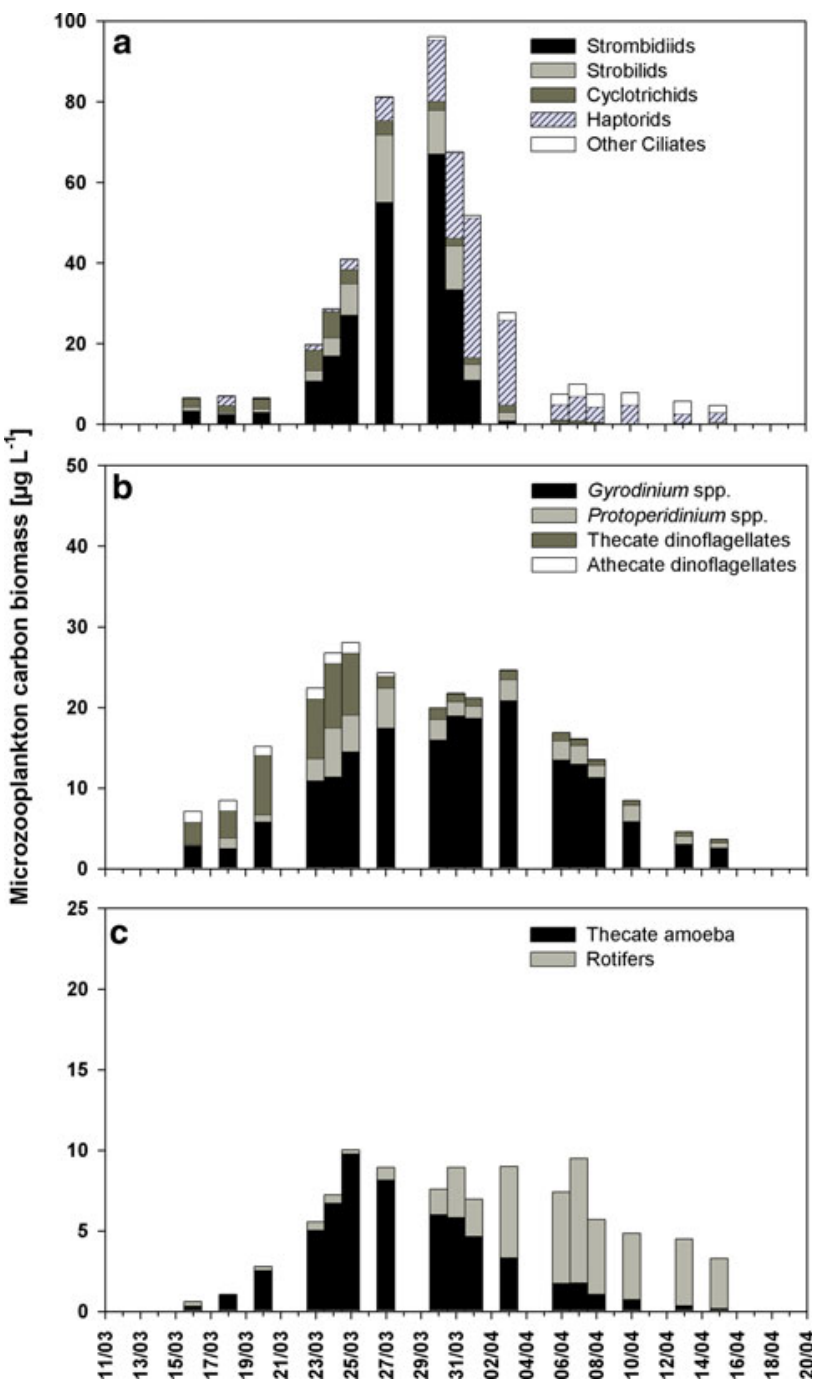

Fig. 3 Microzooplankton species succession during the mesocosm experiment (a ciliates, b dinoflagellates and $\mathbf{c}$ other microzooplankton), mean values of the three mesocosms

onwards reaching a peak on $30.03 .09\left(96 \mu \mathrm{gC} \mathrm{L}^{-1}\right)$ (Fig. 3a). Afterwards, it decreased rapidly to a final $5 \mu \mathrm{gC}$ $\mathrm{L}^{-1}$. Ciliates contributed $27-46 \%$ to the total microzooplankton biomass and dominated the bloom (up to 78\%). A clear succession was found in the community. Until the end of March, Strombidium spp. (S. capitatum, S. cf. emergens, $S$. cf. epidemum, Laboea strobila, Tontonia gracillima and others) dominated. The most important species was $S$. capitatum contributing $92 \%$ to the strombidiids and $64 \%$ to the total ciliate biomass at the ciliate peak. Co-occurring strobilids (Rimostrombidium sp., Lohmanniella oviformis, Leegaardiella sp., Strombidinopsis sp. and others) contributed $6-21 \%$ to the ciliate biomass until the 31.03.09. After the maximum, both genera declined to values below $5 \%$ of total ciliate biomass and strombidiids finally disappeared. Simultaneously, the big haptorid Cyclotrichium sp. started to dominate until the end at $40-67 \%$ of the total 
biomass. Cyclotrichids (Myrionecta rubra, Mesodinium sp. and Askenasia sp.) only initially played a major role. During the final 10 days, the category 'other ciliates' (mainly Acineta sp. and Euplotes spp.) became more important (up to $55 \%$ of ciliate biomass).

\section{Dinoflagellate community}

Dinoflagellate biomass increased directly after the start $\left(\sim 7 \mu \mathrm{gC} \mathrm{L}^{-1}\right)$ at lower rates than those for ciliates (mean 0.15 day $\left.^{-1}\right)$ but peaked already 5 days earlier $(28 \mu \mathrm{gC}$ $\mathrm{L}^{-1}, 25.03 .09$ ) (Fig. 3b). During the following 8 days, it fluctuated on a high level $\left(20-25 \mu \mathrm{gC} \mathrm{L}^{-1}\right)$ and declined afterwards to a final $4 \mu \mathrm{gC} \mathrm{L} \mathrm{L}^{-1}$. Dinoflagellates contributed $21-62 \%$ to the total microzooplankton biomass with a more pronounced role before and after the ciliate peak. Gyrodinium spp. dominated the community at $38-52 \%$ in the first 10 days and thereafter increased to $66-87 \%$ reaching a maximum of $21 \mu \mathrm{gC} \mathrm{L^{-1 }}$ on the 03.04.09. Different Protoperidinium species ( $P$. ovatum, $P$. thorianum, $P$. pellucidum, $P$. cf. leonis, $P$. bipes, $P$. brevipes. $P$. cf pyriforme and others) contributed $2-23 \%$ to the total dinoflagellate biomass. The group 'athecate dinoflagellates' (Warnowia sp., Torodinium sp., Katodinium sp. and small athecate dinoflagellates $<15 \mu \mathrm{m}$ ) contributed $2-19 \%$ to the total biomass until 27.03 .09 and thereafter declined below 1\%. 'Thecate dinoflagellates' (Diplopsalis sp., Dinophysis sp., small thecate dinoflagellates $<15 \mu \mathrm{m}$ and others) contributed $27-48 \%$ to total biomass until the 25.03.09, but declined afterwards to only $6 \%$. The decline in the last two groups was caused mainly by the loss of the smallest dinoflagellates $(<15 \mu \mathrm{m})$.

\section{Other microzooplankton}

Beside ciliates and dinoflagellates, a thecate amoeba and a rotifer (Synchaeta sp.) occurred in the microzooplankton community (Fig. 3c). Both species together contributed 4-30\% to the total microzooplankton biomass with values over $13 \%$ during the last 10 days of the experiment when Synchaeta sp. became more abundant, whereas the parasitic thecate amoeba (mainly attached to Chaetoceros spp.) dominated this group until the end of March.

\section{Microzooplankton grazing and selectivity}

The microzooplankton community showed carbon-specific grazing rates $g_{c}$ between 0.006 and $0.014(\mu \mathrm{gC}$ predator) ${ }^{-1}$ day $^{-1}$ during the grazing experiments (Fig. 4). All groups of phytoplankton were grazed, while we detected different selectivity patterns for different taxa. Detailed information is given in Tables 1,2 and on prey taxon level in the "Appendix".

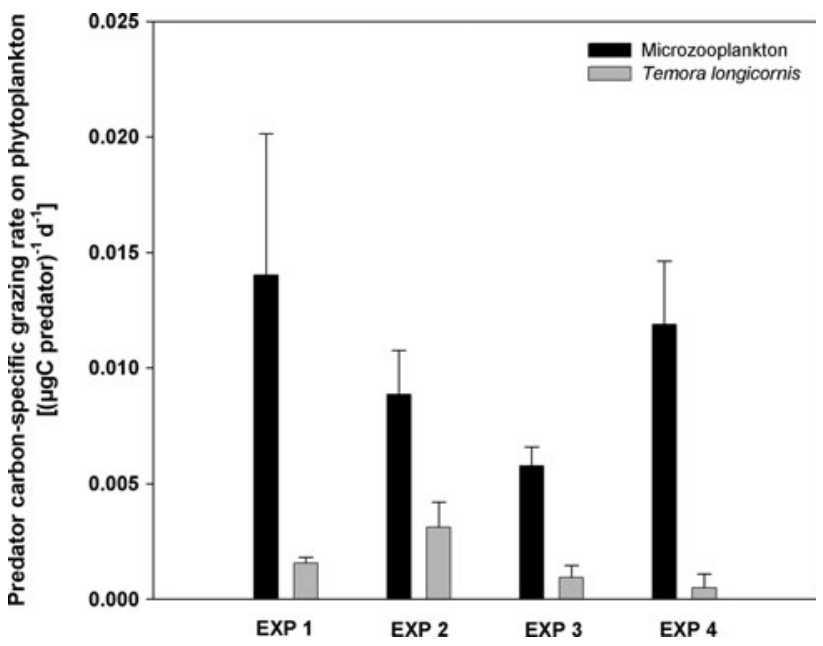

Fig. 4 Predator carbon-specific grazing rates $g_{c}$ of microzooplankton and Temora longicornis grazing on phytoplankton during the four experiments, error bars correspond to one standard error $(n=36$ for microzooplankton, $n=9$ for $T$. longicornis)

Before the bloom (experiment 1), dinoflagellates dominated the grazer biomass (62\%) followed by ciliates $(27 \%)$. They displayed maximal growth rates of $\sim 0.3$ day $^{-1}$. Saturated feeding was detected in 12 out of 20 phytoplankton prey species accompanied by low grazer biomass $(30 \mu \mathrm{g}$ $\mathrm{L}^{-1}$ ) (“Appendix", Table 5). However, microzooplankton showed a total grazing rate $g$ of $0.43 \mathrm{day}^{-1}$ (Table 1) and the highest carbon-specific ingestion rate $I_{c}$ of $1.57 \mu \mathrm{gC}$ prey $\mu \mathrm{gC}$ predator $^{-1}$ day $^{-1}$ on total phytoplankton among the four experiments, leading to a total daily ingestion $I_{\text {total }}$ of $47.65 \mu \mathrm{gC} \mathrm{L}{ }^{-1}$ day $^{-1}$ (Table 2) on the community level. Microzooplankton grazed 53\% of the phytoplankton initial stock $\left(P_{i}\right)$ and $223 \%$ of the total potential production $\left(P_{p}\right)$ (Table 1) due to the lowest instantaneous growth $\mu_{0}$ $\left(0.17 \mathrm{day}^{-1}\right)$ of prey in our four experiments. Based on the index $E^{*}$ microzooplankton clearly selected the groups Chaetoceros spp. and Thalassiosira spp. (Table 2).

At the phytoplankton peak (experiment 2), total grazer biomass $\left(74 \mu \mathrm{g} \mathrm{L}^{-1}\right)$ was more than twice as high as during the pre-bloom experiment and shares of dinoflagellate and ciliate biomass were almost equal. Ciliates displayed the highest growth rates of $0.37 \mathrm{day}^{-1}$ in this phase. The community showed a total $I_{c}$ of $1.00 \mu \mathrm{gC}$ prey $\mu \mathrm{gC}$ predator $^{-1}$ day $^{-1}$ (Table 2) on all phytoplankton and a total grazing rate $g$ of 0.66 day $^{-1}$ (Table 1$)$. As $\mu_{0}\left(0.77\right.$ day $^{-1}$ ) was higher than $g, 93 \%$ of $P_{i}$ and $90 \%$ of $P_{p}$ (Table 1) were grazed. Food selectivity reflected the high grazer diversity and was spread over all categories of phytoplankton resulting in a total daily ingestion $I_{\text {total }}$ of $74.58 \mu \mathrm{gC}$ $\mathrm{L}^{-1}$ day $^{-1}$ (Table 2). On group level microzooplankton selected flagellates and Chaetoceros spp. (Table 2).

The early post-bloom phase (experiment 3 ) was characterised by the highest grazer biomass of all experiments 
Table 1 Microzooplankton grazing $g\left(\right.$ day $\left.^{-1}\right)$, phytoplankton growth rates $k\left(\right.$ day $\left.^{-1}\right)$ and instantaneous growth rate values $\mu_{o}\left(\right.$ day $\left.^{-1}\right)$ from bottles without added nutrients, percentage of initial stock $P_{i}(\%)$ and potential production $P_{p}(\%)$ grazed as determined in four dilution experiments for different phytoplankton groups

\begin{tabular}{|c|c|c|c|c|c|c|c|c|c|c|c|c|c|c|}
\hline \multirow[t]{3}{*}{ Phytoplankton } & \multicolumn{14}{|c|}{ Microzooplankton } \\
\hline & \multicolumn{7}{|c|}{ Experiment 1 (MMC $\left.30.33 \mu \mathrm{gC} \mathrm{L}^{-1}\right)$} & \multicolumn{7}{|c|}{ Experiment $2\left(\mathrm{MMC} 74.47 \mu \mathrm{gC} \mathrm{L}^{-1}\right)$} \\
\hline & $k$ & $P$ & $g$ & $P$ & $\mu_{O}$ & $P_{i}$ & $P_{p}$ & $k$ & $P$ & $g$ & $P$ & $\mu_{O}$ & $P_{i}$ & $P_{p}$ \\
\hline Chaetoceros spp. & 0.46 & & 0.60 & $*$ & 0.40 & 83 & 139 & 1.15 & $* * * *$ & 1.27 & $* * * *$ & 1.50 & 256 & 92 \\
\hline Rhizosolenia spp. & 0.25 & & 0.28 & $* *$ & -0.01 & 32 & 0 & 0.70 & $* * * *$ & 0.76 & $* * * *$ & 0.78 & 114 & 98 \\
\hline Thalassiosira spp. & 0.53 & $* * * *$ & 0.56 & $* *$ & 0.42 & 75 & 124 & 0.60 & $* * * *$ & 0.63 & $* * * *$ & 0.85 & 88 & 82 \\
\hline Flagellates & 0.21 & & 0.19 & $* *$ & -0.47 & 21 & 0 & 0.58 & $* * *$ & 0.84 & * & 0.74 & 132 & 109 \\
\hline Other diatoms & 0.02 & & -0.10 & $*$ & -0.47 & 0 & 0 & 0.35 & $* * * *$ & 0.41 & $* *$ & 0.27 & 51 & 141 \\
\hline \multirow[t]{2}{*}{ Total phytoplankton } & 0.41 & * & 0.43 & $*$ & 0.17 & 53 & 223 & 0.80 & $* * * *$ & 0.66 & $* * *$ & 0.77 & 93 & 90 \\
\hline & \multicolumn{7}{|c|}{ Experiment 3 (MMC $93.82 \mu \mathrm{gC} \mathrm{L}^{-1}$ ) } & \multicolumn{7}{|c|}{ Experiment 4 (MMC $33.12 \mu \mathrm{gC} \mathrm{L}^{-1}$ ) } \\
\hline Chaetoceros spp. & 0.49 & $* * *$ & 0.52 & $*$ & 0.41 & 68 & 121 & 0.80 & $* * * *$ & 0.70 & $* * * *$ & 1.27 & 102 & 70 \\
\hline Rhizosolenia spp. & 0.43 & $* * * *$ & 0.42 & $* * * *$ & 0.65 & 52 & 72 & 0.51 & $* * * *$ & 0.49 & $* * * *$ & 0.76 & 64 & 73 \\
\hline Thalassiosira spp. & 0.14 & $* * *$ & 0.15 & $*$ & 0.19 & 16 & 78 & 0.68 & $* * * *$ & 0.69 & $* * * *$ & 0.89 & 100 & 85 \\
\hline Flagellates & 0.58 & $* * * *$ & 0.53 & $* * *$ & 0.72 & 70 & 81 & -0.20 & & 0.10 & & -0.47 & 10 & 0 \\
\hline Other diatoms & 0.38 & $* * *$ & 0.37 & $*$ & 0.32 & 45 & 114 & 0.72 & $* * * *$ & 0.71 & $* * * *$ & 0.98 & 103 & 81 \\
\hline Total phytoplankton & 0.39 & $* * * *$ & 0.54 & $* * * *$ & 0.65 & 72 & 87 & 0.41 & $* * * *$ & 0.39 & $* * *$ & 0.52 & 48 & 80 \\
\hline
\end{tabular}

Food saturation given in italic values; negative $P_{i}$ and $P_{p}$ values resulting from negative $g\left(P_{i}\right)$ or $\mu_{o}\left(P_{p}\right)$ were set to zero, also positive $P_{p}$ values resulting from negative $g$ and $\mu_{o}$

$M M C$ mean microzooplankton carbon biomass

$P$ values from linear regression analysis of apparent phytoplankton growth against dilution factor $(n=36)$, * $P<0.05$; ** $P<0.01$; $* * * P<0.001 ; * * * * P<0.0001$

Table 2 Microzooplankton carbon-specific filtration rates $F_{c}\left(\mathrm{~mL} \mu \mathrm{gC}\right.$ predator ${ }^{-1}$ day $^{-1}$ ) and carbon-specific ingestion rates $I_{c}(\mu \mathrm{gC}$ prey $\mu \mathrm{gC}$ predator $^{-1}$ day $\left.^{-1}\right)$, total ingestion rates of the microzooplankton community $I_{\text {total }}\left(\mu \mathrm{gC}\right.$ prey $\mathrm{L}^{-1}$ day $\left.^{-1}\right)$ and electivity $E^{*}(-)$ for different phytoplankton groups

\begin{tabular}{|c|c|c|c|c|c|c|c|c|c|c|}
\hline \multirow[t]{3}{*}{ Phytoplankton } & \multicolumn{10}{|c|}{ Microzooplankton } \\
\hline & \multicolumn{5}{|c|}{ Experiment $1\left(\mathrm{MMC} 30.33 \mu \mathrm{gC} \mathrm{L}^{-1}\right)$} & \multicolumn{5}{|c|}{ Experiment $2\left(\mathrm{MMC} 74.47 \mu \mathrm{gC} \mathrm{L}^{-1}\right)$} \\
\hline & $F_{c}$ & $P$ & $I_{c}$ & $P$ & $E^{*}$ & $F_{c}$ & $P$ & $I_{c}$ & $P$ & $E^{*}$ \\
\hline Chaetoceros spp. & 19.88 & $*$ & 0.30 & $*$ & 0.30 & 17.04 & $* * * *$ & 0.16 & $* * * *$ & 0.24 \\
\hline Rhizosolenia spp. & 9.11 & $* *$ & 0.33 & $* *$ & -0.08 & 10.22 & $* * * *$ & 0.41 & $* * * *$ & -0.01 \\
\hline Thalassiosira spp. & 18.50 & $* *$ & 0.73 & $* *$ & 0.26 & 8.46 & $* * * *$ & 0.35 & $* * * *$ & -0.11 \\
\hline Flagellates & 6.35 & $* *$ & 0.10 & $* *$ & -0.26 & 11.31 & $*$ & 0.11 & $*$ & 0.04 \\
\hline Other diatoms & -3.46 & * & -0.02 & $*$ & -1.00 & 5.49 & $* *$ & 0.03 & $* *$ & -0.31 \\
\hline Total phytoplankton & 14.02 & * & 1.57 & $*$ & & 8.85 & $* * *$ & 1.00 & $* * *$ & \\
\hline \multirow[t]{2}{*}{ Related to total microzooplankton $\mathrm{C} \mathrm{L}^{-1}$} & & $I_{\text {total }}$ & 47.65 & & & & $I_{\text {total }}$ & 74.58 & & \\
\hline & \multicolumn{5}{|c|}{ Experiment 3 (MMC $93.82 \mu \mathrm{gC} \mathrm{L}^{-1}$ ) } & \multicolumn{5}{|c|}{ Experiment 4 (MMC $33.12 \mu \mathrm{gC} \mathrm{L}^{-1}$ ) } \\
\hline Chaetoceros spp. & 5.53 & $*$ & 0.08 & $*$ & 0.13 & 21.19 & $* * * *$ & 0.17 & $* * * *$ & 0.13 \\
\hline Rhizosolenia spp. & 4.48 & $* * * *$ & 0.16 & $* * * *$ & 0.03 & 14.93 & $* * * *$ & 0.39 & $* * * *$ & -0.04 \\
\hline Thalassiosira spp. & 1.59 & * & 0.08 & $*$ & -0.46 & 20.94 & $* * * *$ & 0.49 & $* * * *$ & 0.13 \\
\hline Flagellates & 5.68 & $* * *$ & 0.09 & $* * *$ & 0.14 & 2.92 & & 0.06 & & -0.70 \\
\hline Other diatoms & 3.98 & $*$ & 0.01 & $*$ & -0.03 & 21.45 & $* * * *$ & 0.02 & $* * * *$ & 0.14 \\
\hline Total phytoplankton & 5.77 & $* * * *$ & 0.62 & $* * * *$ & & 11.89 & $* * *$ & 0.97 & $* * *$ & \\
\hline Related to total microzooplankton $\mathrm{C} \mathrm{L}^{-1}$ & & $I_{\text {total }}$ & 58.02 & & & & $I_{\text {total }}$ & 32.13 & & \\
\hline
\end{tabular}

Positive selection given in italic values

$M M C$ mean microzooplankton carbon biomass

$P$ values are the same as for the grazing rates of microzooplankton, $* P<0.05 ; * * P<0.01$; *** $P<0.001 ; * * * *<0.0001$ 
(94 $\mu \mathrm{g} \mathrm{L}^{-1}$ ) and ciliates clearly dominated the community (69\%). Microzooplankton grazed $72 \%$ of $P_{i}$ and $87 \%$ of $P_{p}$ of the total phytoplankton community at a rate of 0.54 day $^{-1}(g)$ (Table 1). Phytoplankton displayed a higher instantaneous growth rate $\mu_{0}\left(0.65 \mathrm{day}^{-1}\right)$ than in the fertilised incubation bottles $\left(k=0.39 \mathrm{day}^{-1}\right) . I_{c}(0.62 \mu \mathrm{gC}$ prey $\mu \mathrm{gC}$ predator ${ }^{-1} \mathrm{day}^{-1}$ ) on total phytoplankton was the lowest detected in our experiments leading to a total daily ingestion $I_{\text {total }}$ of $58.02 \mu \mathrm{gC} \mathrm{L} \mathrm{L}^{-1} \mathrm{day}^{-1}$ of the microzooplankton (Table 2). Microzooplankton selected for flagellates (except flagellates $5 \mu \mathrm{m}$, see "Appendix"), Rhizosolenia spp. and Chaetoceros spp. (Table 2).

In the late post-bloom phase (experiment 4), grazer biomass $\left(33 \mu \mathrm{g} \mathrm{L}^{-1}\right)$ was as low as before the bloom and saturated feeding in 5 phytoplankton species was detected ("Appendix", Table 5). Besides the now dominating dinoflagellates, rotifers became as important as ciliates. The community grazed $48 \%$ of $P_{i}$ and $80 \%$ of $P_{p}$ at a rate of 0.39 day $^{-1}(g)$ (Table 1$) . I_{c}(0.97 \mu \mathrm{gC}$ prey $\mu \mathrm{gC}$ predator $^{-1}$ day $^{-1}$ ) increased to a value similar to experiment 2 resulting in a total daily ingestion $I_{\text {total }}$ of $32.13 \mu \mathrm{gC}$ $\mathrm{L}^{-1}$ day $^{-1}$. Again instantaneous growth $\mu_{0}\left(0.52\right.$ day $\left.^{-1}\right)$ of the phytoplankton exceeded the growth in fertilised bottles $\left(0.41\right.$ day $\left.^{-1}\right)$ (Table 1$)$. Selectivity was similar to experiment 1 , whereas also the category 'other diatoms' was selected.

\section{Temora longicornis grazing and selectivity}

The T. longicornis $\left(108.7 \mu \mathrm{gC} \mathrm{L} \mathrm{L}^{-1}\right)$ biomass added was always higher than the microzooplankton biomass in the experiments. Even so, the copepod species had a much lower grazing impact on the phytoplankton community (Fig. 4) than the microzooplankton $\left(g_{c}: 0.0005-0.003(\mu \mathrm{gC}\right.$ predator $)^{-1}$ day $^{-1}$ ). During the course of the experiments, $T$. longicornis switched its diet gradually from a phytoplankton-dominated towards a microzooplankton-dominated diet (Fig. 5a; Table 4). This was reflected in $E^{*}$ values and a positive selection for microzooplankton in general when compared to phytoplankton prey, with the exception of the phytoplankton peak experiment (Fig. 5b; Table 4). However, on a taxon level some phytoplankters were also positively selected (see "Appendix" for details). Detailed information on individual and carbon-based grazing parameters of $T$. longicornis is given on prey group level in Tables 3, 4.

Before the bloom (experiment 1), the copepods showed a grazing rate of $0.17 \mathrm{day}^{-1}$ (Table 3) and ingested $17.87 \mu \mathrm{gC} \mathrm{L}^{-1} \mathrm{day}^{-1}$ of the total phytoplankton community $\left(I_{\text {total }}\right)$ at a carbon-specific ingestion rate $I_{c}$ of $0.16 \mu \mathrm{gC}$ prey $\mu \mathrm{gC}$ predator ${ }^{-1} \mathrm{day}^{-1}$ (Table 4) leading to $18 \%$ reduction of $P_{i}$ and $100 \%$ of $P_{p}$ (Table 3). Per capita filtration rate $F_{i}$ was $15.51 \mathrm{~mL}^{\text {Ind. }}{ }^{-1}$ day $^{-1}$ and one copepod ingested 1,644 ngC Ind. ${ }^{-1}$ day $^{-1}$ of phytoplankton
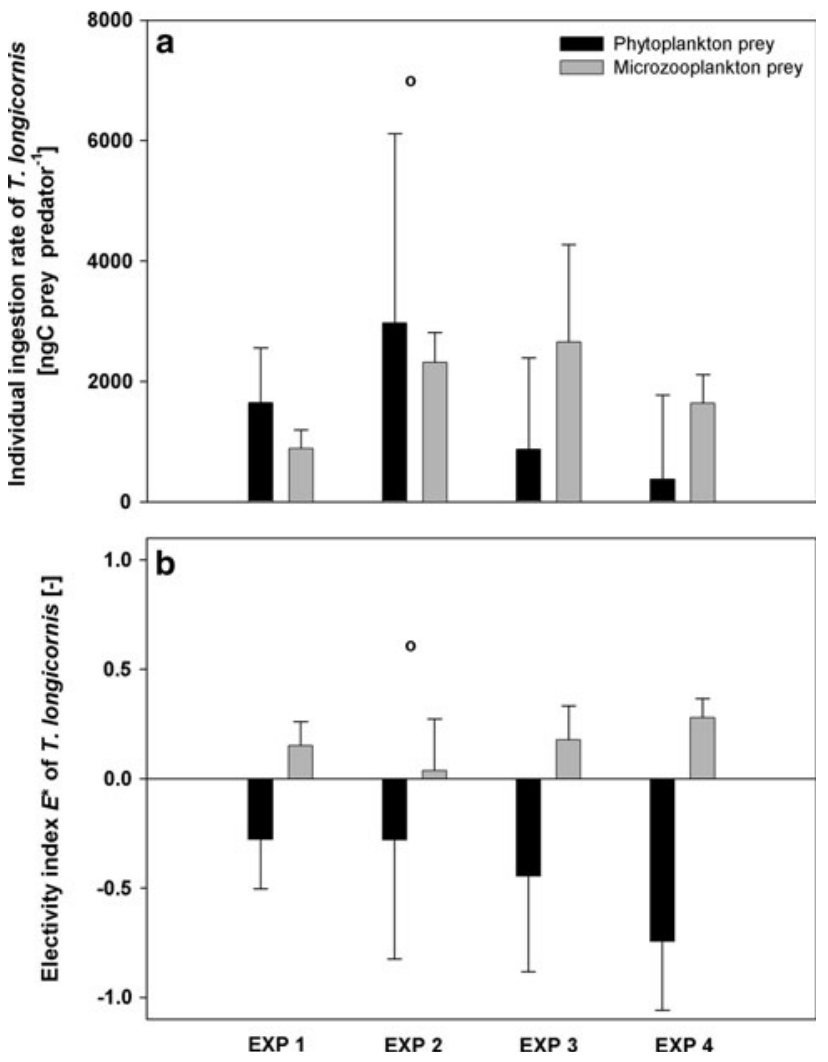

Fig. 5 a Individual ingestion rate $I_{i}$ and $\mathbf{b}$ electivity index $E^{*}$ of Temora longicornis for phytoplankton and microzooplankton prey, 'o' marks experiment with insignificant differences of $I_{i}$ and $E^{*}$ between both prey groups, error bars correspond to one standard deviation $(n=9)$

$\left(I_{i}\right.$, Table 4). Due to the lower biomass of microzooplankton prey, the copepods reached a higher $g\left(0.40 \mathrm{day}^{-1}\right)$ and they ingested $9.58 \mu \mathrm{gC} \mathrm{L}^{-1}$ day $^{-1}$ $\left(I_{\text {total }}\right)$ of the microzooplankton community at an $I_{c}$ of $0.09 \mu \mathrm{gC}$ prey $\mu \mathrm{gC}$ predator $^{-1} \mathrm{day}^{-1}$, leading to a $49 \%$ decrease of $P_{i}$ and $174 \%$ of $P_{p}$ (Table 3). One copepod cleared $36.72 \mathrm{~mL}$ Ind. $^{-1}$ day $^{-1}$ and ingested $881 \mathrm{ngC}$ Ind. ${ }^{-1}$ day $^{-1}$ in form of microzooplankton (Table 4).

Only at the bloom peak (experiment 2), electivity for microzooplankton prey was insignificant (Fig. 5). The copepods ingested the highest amount of biomass during our experiments $\left(57.50 \mu \mathrm{gC} \mathrm{L} \mathrm{L}^{-1}\right.$ day $^{-1}, \quad I_{\text {total }}$ phytoplankton + microzooplankton, Table 4 ) and showed grazing rates of 0.34 day $^{-1}$ for phytoplankton and 0.43 day $^{-1}$ for microzooplankton (Table 3), at carbon-specific ingestion rates $I_{c}$ of 0.30 and $0.23 \mu \mathrm{gC}$ prey $\mu \mathrm{gC}$ predator $^{-1}$ day $^{-1}$, respectively (Table 4). Copepods grazed $40 \%$ of phytoplankton $P_{i}$ (highest value for T. longicornis) and 54\% of its $P_{p}$ and 53\% of the microzooplankton $P_{i}$ and $147 \%$ of its $P_{p}$. Per capita filtration rate $F_{i}$ was $31.21 \mathrm{~mL}$ Ind. $^{-1}$ day $^{-1}$ for phytoplankton and $39.33 \mathrm{~mL}$ Ind. $^{-1}$ day $^{-1}$ for microzooplankton and an individual ingested 2,974 ngC Ind. ${ }^{-1}$ day $^{-1}$ phytoplankton and 2,316 ngC Ind. ${ }^{-1}$ day $^{-1}$ microzooplankton. 
Table 3 Temora longicornis grazing $g\left(\right.$ day $^{-1}$ ), phytoplankton and microzooplankton growth rates $k\left(\right.$ day $\left.^{-1}\right)$ and phytoplankton instantaneous growth rate values $\mu_{o}\left(\right.$ day $\left.^{-1}\right)$ from bottles without added nutrients, percentage of initial stock $P_{i}(\%)$ and potential production $P_{p}(\%)$ grazed as determined in four grazing experiments for different prey groups

\begin{tabular}{|c|c|c|c|c|c|c|c|c|c|c|c|c|c|c|}
\hline & \multicolumn{14}{|c|}{ Temora longicornis $\left(\mathrm{TC} 108.70 \mu \mathrm{gC} \mathrm{L}^{-1}\right.$ ) } \\
\hline & \multicolumn{7}{|c|}{ Experiment 1} & \multicolumn{7}{|c|}{ Experiment 2} \\
\hline & $k$ & $P$ & $g$ & $P$ & $\mu_{0}$ & $P_{i}$ & $P_{p}$ & $k$ & $P$ & $g$ & $P$ & $\mu_{0}$ & $P_{i}$ & $P_{p}$ \\
\hline \multicolumn{15}{|l|}{ Phytoplankton } \\
\hline Chaetoceros spp. & -0.13 & & 0.34 & $* * *$ & 0.40 & 40 & 88 & 0.24 & $*$ & 0.47 & $*$ & 1.50 & 60 & 48 \\
\hline Rhizosolenia spp. & -0.02 & & 0.13 & $*$ & -0.01 & 14 & 0 & 0.01 & & 0.20 & & 0.78 & 23 & 34 \\
\hline Thalassiosira spp. & 0.00 & & 0.21 & $* *$ & 0.42 & 23 & 54 & 0.31 & $* *$ & 0.56 & $*$ & 0.85 & 74 & 74 \\
\hline Flagellates & 0.01 & & 0.05 & & -0.47 & 5 & 0 & -0.26 & $*$ & 0.08 & & 0.74 & 8 & 14 \\
\hline Other diatoms & 0.11 & & -0.05 & & -0.47 & 0 & 0 & -0.16 & & 0.02 & & 0.27 & 2 & 9 \\
\hline Total phytoplankton & -0.01 & & 0.17 & $* * *$ & 0.17 & 18 & 100 & 0.13 & $*$ & 0.34 & $*$ & 0.77 & 40 & 54 \\
\hline \multicolumn{15}{|l|}{ Microzooplankton } \\
\hline Gyrodinium spp. & -0.02 & & 1.11 & $* *$ & & 203 & 0 & 0.32 & $* * * *$ & 0.27 & $* * *$ & & 31 & 86 \\
\hline Protoperidinium spp. & 0.20 & & 1.32 & $* * *$ & & 275 & 406 & 0.21 & & 0.33 & $* *$ & & 39 & 150 \\
\hline other athecate dinoflagellates & 0.03 & & 0.18 & & & 20 & 497 & -0.01 & & 0.27 & $*$ & & 30 & 0 \\
\hline other thecate dinoflagellates & 0.39 & $* * * *$ & -0.03 & & & 0 & 0 & -0.43 & $* * * *$ & 0.20 & $*$ & & 23 & 0 \\
\hline Strombidium spp. & 0.30 & $*$ & 1.18 & $* * *$ & & 224 & 267 & 0.46 & $* * * *$ & 0.79 & $* * * *$ & & 121 & 148 \\
\hline Strobilidium spp. & 0.26 & & 0.92 & $*$ & & 150 & 262 & 0.66 & $* * *$ & 0.36 & $* *$ & & 43 & 62 \\
\hline Cyclotrichids & 0.18 & & 0.95 & $* *$ & & 158 & 374 & -0.32 & & 0.16 & & & 18 & 0 \\
\hline Haptorids & -0.28 & & 0.93 & $*$ & & 154 & 0 & 0.39 & & 0.35 & & & 42 & 91 \\
\hline Other ciliates & -0.03 & & 0.04 & & & 4 & 0 & 0.17 & & 0.58 & $*$ & & 79 & 289 \\
\hline Thecate amoeba sp. & 0.16 & & 0.32 & $* * *$ & & 38 & 191 & 0.25 & $* *$ & 0.23 & $*$ & & 26 & 93 \\
\hline Rotifers & 0.00 & & - & & & - & - & - & & - & & & - & - \\
\hline \multirow[t]{2}{*}{ Total microzooplankton } & 0.21 & $* * * *$ & 0.40 & $* * * *$ & & 49 & 174 & 0.27 & $* * * *$ & 0.43 & $* * * *$ & & 53 & 147 \\
\hline & \multicolumn{7}{|c|}{ Experiment 3} & \multicolumn{7}{|c|}{ Experiment 4} \\
\hline \multicolumn{15}{|l|}{ Phytoplankton } \\
\hline Chaetoceros spp. & -0.02 & & 0.23 & $*$ & 0.41 & 25 & 61 & 0.28 & $*$ & 0.21 & & 1.27 & 23 & 26 \\
\hline Rhizosolenia spp. & -0.09 & & 0.03 & & 0.65 & 3 & 5 & 0.13 & & 0.11 & & 0.76 & 12 & 20 \\
\hline Thalassiosira spp. & -0.35 & & -0.03 & & 0.19 & 0 & 0 & 0.08 & & 0.21 & $*$ & 0.89 & 24 & 32 \\
\hline Flagellates & -0.06 & & 0.23 & $* *$ & 0.72 & 26 & 40 & -0.28 & $* *$ & -0.24 & $* * *$ & -0.47 & 0 & 0 \\
\hline Other diatoms & -0.14 & & 0.40 & $*$ & 0.32 & 49 & 120 & 0.23 & $* *$ & 0.21 & $*$ & 0.98 & 24 & 31 \\
\hline Total phytoplankton & -0.18 & & 0.10 & & 0.65 & 11 & 20 & 0.04 & & 0.05 & & 0.52 & 6 & 13 \\
\hline \multicolumn{15}{|l|}{ Microzooplankton } \\
\hline Gyrodinium spp. & 0.05 & & 0.21 & $* * *$ & & 24 & 425 & -0.11 & & 0.66 & $* *$ & & 93 & 0 \\
\hline Protoperidinium spp. & 0.12 & & 0.76 & $* *$ & & 114 & 487 & -0.06 & & 0.53 & $* *$ & & 70 & 0 \\
\hline Other athecate dinoflagellates & 0.05 & & 0.22 & & & 25 & 418 & - & & - & & & - & - \\
\hline Other thecate dinoflagellates & -0.14 & & 0.14 & & & 15 & 0 & -0.35 & & 0.07 & & & 7 & 0 \\
\hline Strombidium spp. & -1.61 & $* * * *$ & 0.15 & & & 16 & 0 & 1.30 & & 0.32 & & & 37 & 37 \\
\hline Strobilidium spp. & -0.61 & $* * * *$ & 0.22 & & & 25 & 0 & -0.21 & & 0.40 & & & 50 & 0 \\
\hline Cyclotrichids & 0.01 & & 0.59 & * & & 80 & 3066 & -0.46 & & 0.49 & & & 63 & 0 \\
\hline Haptorids & 0.87 & $* *$ & 0.54 & $* *$ & & 72 & 72 & -0.52 & & 2.48 & & & 1097 & 0 \\
\hline Other ciliates & 0.54 & & 0.44 & & & 56 & 85 & -0.11 & & 0.10 & & & 11 & 0 \\
\hline Thecate amoeba sp. & -0.11 & & 0.13 & $*$ & & 13 & 0 & -0.05 & & 0.25 & $* * *$ & & 29 & 0 \\
\hline Rotifers & 0.02 & & 0.33 & & & 39 & 1183 & 0.03 & & 1.84 & $* * *$ & & 529 & 3,349 \\
\hline Total microzooplankton & 0.07 & & 0.38 & $* * *$ & & 46 & 478 & -0.04 & & 0.78 & $* * * *$ & & 118 & 0 \\
\hline
\end{tabular}

Negative $P_{i}$ and $P_{p}$ values resulting from negative $g\left(P_{i}\right)$ or $\mu_{o} / k\left(P_{p}\right)$ were set to zero, also positive $P_{p}$ values resulting from negative $g$ and $\mu_{o} / k$ $T C$ total T. longicornis carbon biomass

$P$ values derived from $t$ tests against zero, $* P<0.05$; ** $P<0.01$; *** $P<0.001$; **** $P<0.0001$ 
Table 4 Temora longicornis individual filtration $F_{i}\left(\mathrm{~mL} \mathrm{Ind.}^{-1}\right.$ day ${ }^{-1}$ ) and ingestion rates $I_{i}\left(\mathrm{ngC} \mathrm{Ind}^{-1}\right.$ day $^{-1}$ ) as well as carbon-specific filtration rates $F_{c}\left(\mathrm{~mL} \mu \mathrm{gC}\right.$ predator ${ }^{-1}$ day $\left.^{-1}\right)$ and carbon-specific ingestion rates $I_{c}\left(\mu \mathrm{gC}\right.$ prey $\mu \mathrm{gC}$ predator $^{-1}$ day $^{-1}$ ), total ingestion rates $I_{\text {total }}$ $\left(\mu \mathrm{gC}\right.$ prey $\left.\mathrm{L}^{-1} \mathrm{day}^{-1}\right)$ and electivity $E^{*}(-)$ for different prey groups

Temora longicornis (TC $108.70 \mu \mathrm{gC} \mathrm{L}^{-1}$ )
Experiment 1

$F_{i}-F_{c}+I_{i}+I_{c}+T^{2}+E^{*}$

Experiment 2

\begin{tabular}{llllllll}
\hline$F_{i}$ & $F_{c}$ & $P$ & $I_{i}$ & $I_{c}$ & $P$ & $E^{*}$ & $P$
\end{tabular}

\section{Phytoplankton}

oceros spp.

31.19

11.94

18.89

Thalassiosira spp.

Flagellates

Other diatoms

Total phytoplankton

Related to total copepod $\mathrm{C} \mathrm{L}^{-1}$

\section{Microzooplankton}

Gyrodinium spp.

Protoperidinium spp.

Other athecate dinoflagellates

Other thecate

dinoflagellates

Strombidium spp.

Strobilidium spp.

Cyclotrichids

Haptorids

Other ciliates

Thecate amoeba sp.

Rotifers

Total

microzooplankton

Related to total copepod $\mathrm{C} \mathrm{L}^{-1}$

$-4.97$

15.51

\begin{abstract}
102.01
\end{abstract}
16.51

108.29

84.30

87.07

85.78

3.25

29.76

$-$

36.72

\subsection{4}

$$
3.12 * * *
$$

403.04

0.

$1.89 * *$

689.01

0.42

$-0.50$

$1.55 * * *$

32.41

$-0.0023$

$1643.64 \quad 0.164$

$I_{\text {total }} \quad 17.87$

$10.20 * *$

$12.17 * * *$

1.65

$-2.36 \quad-0.24$
$10.83 * * *$

$8.43 *$

$8.71 * *$

$8.58 *$

0.33

$2.98 * * *$

$-$

$3.67 * * * *$
263.25

79.97

20.68

$-21.03-0.0021$

251.16

42.07

179.21

7.02

$60.68 \quad 0.0061 * * * \quad-0.26$

-
-

$880.910 .0881 \quad * * * * \quad 0.15 * *$

$I_{\text {total }} \quad 9.58$

$\begin{array}{rll}43.29 & 4.33 & * \\ 18.74 & 1.87 & \\ 51.06 & 5.11 & * \\ 7.09 & 0.71 & \\ 1.92 & 0.19 & \\ 31.21 & 3.12 & *\end{array}$

333.07

688.94

1784.99

61.63

27.57

2973.72

$I_{\text {total }} \quad 32.32$
$0.0333 *-0.03$

$0.0689-0.30$

$0.1785 * \quad-0.01$

$0.0062-0.61 * *$

$0.0028-0.62 * *$

$0.2974 * \quad-0.28$

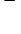

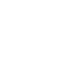

$$
(28)
$$

\begin{tabular}{|c|c|c|c|c|c|c|c|c|c|c|c|c|c|c|c|c|}
\hline \multirow[b]{2}{*}{ Phytoplankton } & \multicolumn{8}{|c|}{ Experiment 3} & \multicolumn{8}{|c|}{ Experiment 4} \\
\hline & & & & & & & & & & & & & & & & \\
\hline Chaetoceros spp. & 20.86 & 2.09 & $*$ & 254.93 & 0.0255 & $*$ & -0.34 & & 18.89 & 1.89 & & 131.90 & 0.0132 & & -0.45 & $* *$ \\
\hline Rhizosolenia spp. & 2.32 & 0.23 & & 58.91 & 0.0059 & & -0.68 & $* * *$ & 10.45 & 1.05 & & 233.08 & 0.0233 & & -0.57 & $*$ \\
\hline Thalassiosira spp. & -2.83 & -0.28 & & -259.38 & -0.0259 & & -0.71 & $* *$ & 19.57 & 1.96 & $*$ & 427.66 & 0.0428 & $*$ & -0.32 & \\
\hline Flagellates & 21.36 & 2.14 & $* *$ & 304.11 & 0.0304 & $* *$ & -0.26 & & -22.44 & -2.24 & $* * *$ & -441.30 & -0.0441 & $* * *$ & -1.00 & \\
\hline Other diatoms & 36.43 & 3.64 & $*$ & 43.37 & 0.0043 & $*$ & -0.27 & & 19.45 & 1.94 & $*$ & 18.68 & 0.0019 & $*$ & -0.36 & \\
\hline Total phytoplankton & 9.34 & 0.93 & & 866.25 & 0.0866 & & -0.45 & $*$ & 4.97 & 0.50 & & 370.16 & 0.0370 & & -0.74 & $* * *$ \\
\hline $\begin{array}{l}\text { Related to total } \\
\text { copepod } \mathrm{C} \mathrm{L}^{-1}\end{array}$ & & & & $I_{\text {total }}$ & 9.42 & & & & & & & $I_{\text {total }}$ & 4.02 & & & \\
\hline \multicolumn{17}{|l|}{ Microzooplankton } \\
\hline Gyrodinium spp. & 19.63 & 1.96 & $* * *$ & 320.35 & 0.0320 & $* * *$ & -0.23 & $*$ & 60.35 & 6.04 & $* *$ & 432.87 & 0.0433 & $* * * *$ & 0.15 & \\
\hline Protoperidinium spp. & 70.08 & 7.01 & $* *$ & 102.51 & 0.0103 & $* *$ & 0.23 & & 48.58 & 4.86 & $* *$ & 67.71 & 0.0068 & $*$ & 0.07 & \\
\hline $\begin{array}{l}\text { Other athecate } \\
\text { dinoflagellates }\end{array}$ & 20.41 & 2.04 & & 1.57 & 0.0002 & & -0.55 & & - & - & & - & - & & - & \\
\hline $\begin{array}{l}\text { Other thecate } \\
\text { dinoflagellates }\end{array}$ & 12.98 & 1.30 & & 6.20 & 0.0006 & & -0.29 & & 6.13 & 0.61 & & 2.30 & 0.0002 & & -0.58 & $*$ \\
\hline Strombidium spp. & 13.65 & 1.36 & & -169.88 & -0.0170 & & -0.52 & & 29.25 & 2.93 & & - & - & & - & \\
\hline Strobilidium spp. & 20.27 & 2.03 & & 85.21 & 0.0085 & & -0.33 & & 37.08 & 3.71 & & 1.23 & 0.0001 & & -0.17 & \\
\hline Cyclotrichids & 54.17 & 5.42 & $*$ & 52.98 & 0.0053 & & 0.07 & & 45.00 & 4.50 & & -7.01 & -0.0007 & & -0.08 & \\
\hline
\end{tabular}


Table 4 continued

\begin{tabular}{|c|c|c|c|c|c|c|c|c|c|c|c|c|c|c|c|c|}
\hline \multirow[b]{2}{*}{ Haptorids } & \multicolumn{8}{|c|}{ Experiment 3} & \multicolumn{8}{|c|}{ Experiment 4} \\
\hline & 49.72 & 4.97 & $* *$ & 1116.13 & 0.1116 & & -0.08 & & 228.35 & 22.83 & & 198.93 & 0.0199 & & 0.23 & \\
\hline Other ciliates & 40.91 & 4.09 & & 3.93 & 0.0004 & & 0.01 & & 9.39 & 0.94 & & 29.68 & 0.0030 & & -0.58 & $* *$ \\
\hline Thecate amoeba sp. & 11.55 & 1.16 & $*$ & 46.54 & 0.0047 & $*$ & -0.44 & $*$ & 23.21 & 2.32 & $* * *$ & 39.28 & 0.0039 & $* * *$ & -0.29 & \\
\hline Rotifers & 30.5535 & 3.06 & & 47.86 & 0.0048 & & -0.16 & & 169.13 & 16.91 & $* * *$ & 706.47 & 0.0706 & $* * *$ & 0.55 & $* *$ \\
\hline $\begin{array}{l}\text { Total } \\
\text { microzooplankton }\end{array}$ & 35.02 & 3.50 & $* * *$ & 2649.93 & 0.2650 & $* *$ & 0.18 & $*$ & 71.61 & 7.16 & $* * * *$ & 1635.56 & 0.1636 & $* * * *$ & 0.28 & $* * * *$ \\
\hline $\begin{array}{l}\text { Related to total } \\
\text { copepod } \mathrm{C} \mathrm{L}^{-1}\end{array}$ & & & & $I_{\text {total }}$ & 28.80 & & & & & & & $I_{\text {total }}$ & 17.78 & & & \\
\hline
\end{tabular}

Positive selection given in italic values

$T C$ total $T$. longicornis carbon biomass

$P$ values derived from $t$ tests against zero, ${ }^{*} P<0.05$; ** $P<0.01$; *** $P<0.001$; **** $P<0.0001$

In the early post-bloom phase (experiment 3 ), the total amount of carbon ingested by the copepods dropped again to $38.22 \mu \mathrm{gC} \mathrm{L} \mathrm{L}^{-1} \mathrm{day}^{-1}$ (Table 4, I Ital phytoplankton + microzooplankton). They had a grazing rate of $0.10 \mathrm{day}^{-1}$ on phytoplankton and 0.38 day $^{-1}$ on microzooplankton (Table 3) at an $I_{c}$ of 0.09 and $0.27 \mu \mathrm{gC}$ prey $\mu \mathrm{gC}$ predator $^{-1}$ day $^{-1}$, respectively (Table 4). On individual level, a T. longicornis cleared $9.34 \mathrm{~mL}$ Ind. ${ }^{-1}$ day $^{-1}$ for phytoplankton and $35.02 \mathrm{~mL}$ Ind. $^{-1}$ day $^{-1}$ for microzooplankton. Ingestion was $866 \mathrm{ngC} \mathrm{Ind.}{ }^{-1}$ day $^{-1}$ for phytoplankton and 2,650 $\mathrm{ngC} \mathrm{Ind.}^{-1}$ day $^{-1}$ for microzooplankton. The impact of the copepods on the phytoplankton community was once again lower with $11 \%$ of $P_{i}$ and $20 \%$ of $P_{p}$ grazed. On the other hand, T. longicornis grazed $46 \%$ of microzooplankton $P_{i}$ and $478 \%$ of its $P_{p}$.

During the late post-bloom (experiment 4) $I_{\text {total }}$ of the copepods for phytoplankton and microzooplankton further dropped to $21.80 \mu \mathrm{gC} \mathrm{L} \mathrm{L}^{-1} \mathrm{day}^{-1}$ (Table 4). While the copepods had only a grazing rate of 0.05 day $^{-1}(g)$ on phytoplankton at a carbon-specific ingestion rate $I_{c}$ of $0.04 \mu \mathrm{gC}$ prey $\mu \mathrm{gC}$ predator $^{-1} \mathrm{day}^{-1}$, the grazing rate for microzooplankton was $0.78 \mathrm{day}^{-1}(g)$ at an $I_{c}$ of $0.16 \mu \mathrm{gC}$ prey $\mu \mathrm{gC}$ predator ${ }^{-1}$ day $^{-1}$ (Table 3,4$)$. This led in turn to a reduced impact on the phytoplankton community $\left(P_{i}: 6 \%\right.$, $\left.P_{p}: 13 \%\right)$ and an even more pronounced impact on the microzooplankton biomass $\left(P_{i}: 118 \%, P_{p}\right.$ : not defined). Per capita filtration rate of $T$. longicornis on phytoplankton was $4.97 \mathrm{~mL} \mathrm{Ind}^{-1} \mathrm{day}^{-1}$ and one copepod ingested $370 \mathrm{ngC}$ Ind. ${ }^{-1}$ day $^{-1}$ in form of phytoplankton $\left(I_{i}\right.$, Table 4$)$. The highest filtration rate $\left(71.61 \mathrm{~mL}\right.$ Ind. $^{-1}$ day $\left.^{-1}\right)$ measured during our experiments led to an $I_{i}$ of 1,636 ngC Ind. ${ }^{-1}$ day $^{-1}$ for microzooplankton prey.

Microzooplankton predator-prey relationships in the mesocosms

We observed a direct coupling between ciliate and flagellate biomass. This resulted in a strong suppression of flagellate biomass from 26 to $10 \mu \mathrm{gC}$ coincident with the ciliate peak (Fig. 6a). It was most pronounced for thecate and athecate dinoflagellates $<15 \mu \mathrm{m}$, which disappeared totally during the Strombidium capitatum bloom (Fig. 6b). Simultaneously, with the disappearance of both dinoflagellate groups $S$. capitatum started to form cysts and its population collapsed totally within 1 week. With the break down of strombidiid and strobilid biomass at the end of March, a relaxation from grazing pressure enabled the flagellates to regenerate again.

The majority of dinoflagellate species we found in the mesocosms are reported to prefer diatom prey. As diatoms hardly changed in composition, dinoflagellate succession in the mesocosms was not as pronounced as for the ciliates. While two groups disappeared during the first half of our experiment due to predation by ciliates as described earlier, the remaining dinoflagellate community composition remained relatively constant until the end of the experiment.

We also found a strong predator-prey relationship between a thecate amoeba and Chaetoceros spp. The "apparently" parasitic amoeba was found attached only to cells of this genus and followed the bloom development of its host closely, thus declining towards the end of the experiment.

\section{Discussion}

The aim of this study was to explicitly examine the role of microzooplankton grazing on phytoplankton communities during a spring bloom in temperate waters. As waters around Helgoland are subject to strong water column mixing by tidal currents (Hickel et al. 1993), it was necessary to bring a defined water body into the laboratory giving us the opportunity to study the wax and wane of the "same" spring bloom under controlled conditions. Using our mesocosm set-up and excluding mesozooplankton 
Fig. 6 a General development of ciliate and flagellate biomass in the mesocosms, error bars correspond to one standard deviation $(n=3)$, b close predator-prey relationship: development of small dinoflagellates and their predator Strombidium capitatum and Strombidium capitatum cysts, mean values of the three mesocosms
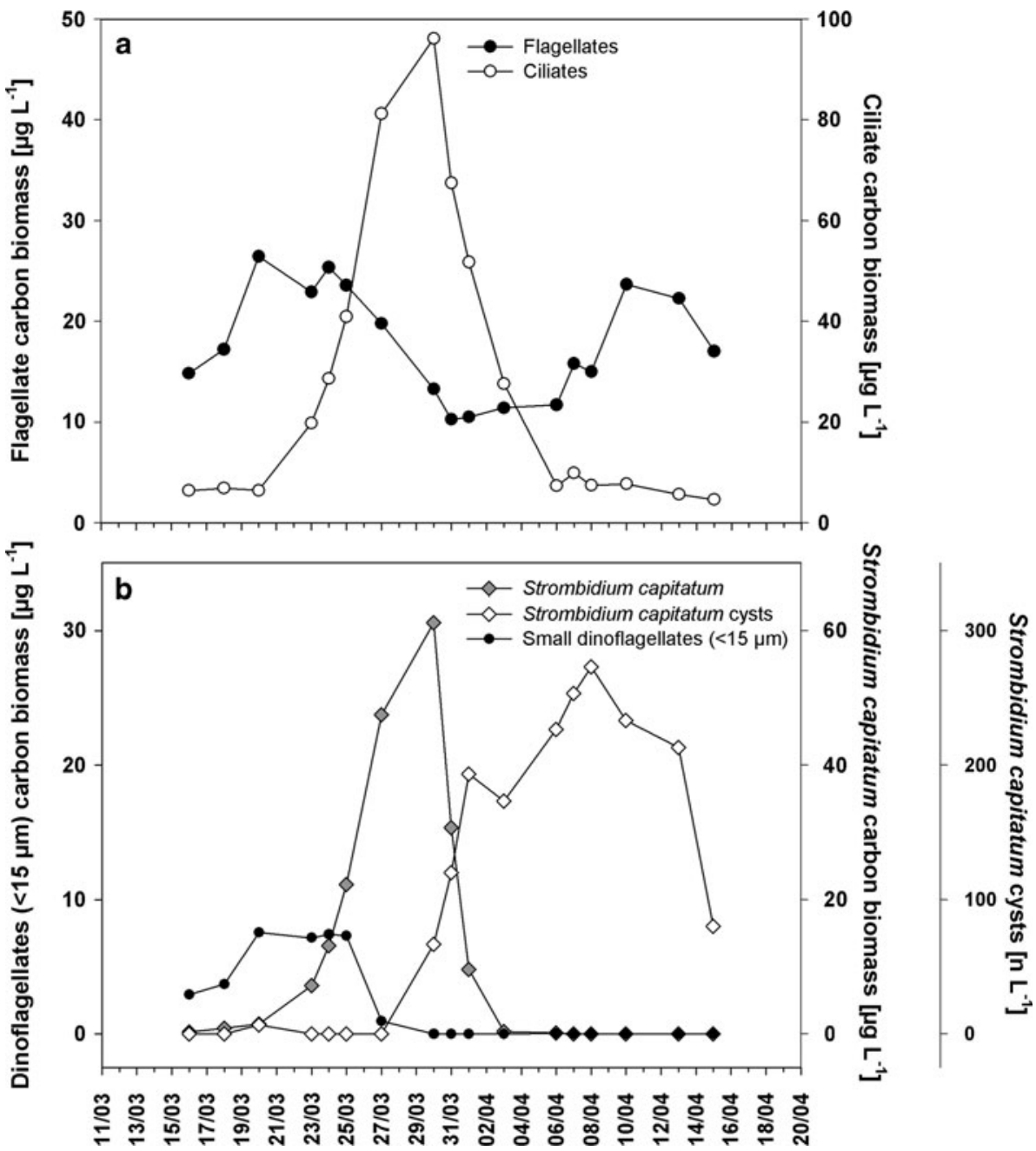

grazers allowed us to follow the plankton spring succession, focusing on top-down control mechanisms on phytoplankton by microzooplankton solely. Furthermore, it allowed us to conduct grazing experiments with microzooplankton as well as copepods at defined points of the phytoplankton spring bloom.

Problems with the estimation of the grazing impact by the dilution technique have been recently discussed (Dolan and McKeon 2005; Landry and Calbet 2005) and the discussion showed that if the restrictions of the dilution technique are recognised, the method has the fundamental advantage of barely altering natural prey and grazer communities and only excluding larger zooplankton. Thus, natural grazing interactions within the microplankton community, as these were our main target, are included in dilution experiments.

Microzooplankton dilution experiments, as applied here, provide us with an alternative to determine grazing rates of microzooplankton by indirect, labour-intensive, and taxonomically selective techniques (Landry and
Hassett 1982; Calbet and Landry 2004). However, methodological restrictions do have to be taken into account (Gallegos 1989; Landry et al. 1995; Dolan et al. 2000; Moigis 2006; Teixeira and Figueiras 2009) most of which are related to the theoretical assumptions (Landry and Hassett 1982) the dilution method is based on. For example, to overcome nonlinear feeding responses due to food saturating conditions, a modified protocol can be used (Paterson et al. 2008; this study). To prevent nutrient limiting conditions in the experimental vessels, often dialysis bags are incubated in situ (Landry 1993; Sommer et al. 2005a, b; Aberle et al. 2007) or nutrients are added in excess to the dilution series (Landry 1993; Fonda Umani et al. 2005; First et al. 2009) in bottle incubations. As we wanted to keep conditions in microzooplankton und copepod grazing experiments comparable, we chose the second alternative (bottle incubations), that was also necessary for correction of trophic cascade effects in the copepod grazing set-up (Nejstgaard et al. 1997, 2001; Fonda Umani et al. 2005). 
Another factor has to be kept in mind when using bottle incubations for studies on microzooplankton. Microzooplankton species, especially ciliates, are highly fragile and sensitive to handling. Filling and mixing procedures during experiments (Gifford 1985; Landry 1993; Broglio et al. 2003) can cause considerable losses, and a high mortality of, e.g., ciliates had been reported previously (up to $80 \%$, Tiselius 1989). This would have, in this study, caused severe underestimations in grazing rates of microzooplankton and also of $T$. longicornis on microzooplankton. To prevent mortality, we applied the new filling method of Löder et al. (2010). Furthermore, microzooplankton survival in the bottles was proven by comparing the microzooplankton communities in the bottles with the mesocosms (see section on "Data analysis"). Although there was always some mortality on a taxon level during incubation, we generally detected positive growth rates (0.07-0.21 day ${ }^{-1}$, Table 3) for the microzooplankton community and only a very low mortality was detected at the decline of the bloom $\left(-0.04 \mathrm{day}^{-1}\right.$, Table 3 , experiment 4). This allowed us to determine realistic grazing estimations and feeding preferences of the microzooplankton community and $T$. longicornis during the mesocosm spring bloom.

Microzooplankton and T. longicornis grazing impact on the phytoplankton bloom

Microzooplankton had a higher grazing potential on phytoplankton throughout the whole period of the phytoplankton spring bloom 2009 in the mesocosms, while copepods only played a secondary role as phytoplankton grazers, which confirms the results of previous studies (e.g., Maar et al. 2004; Fonda Umani et al. 2005). The role of copepods has to be seen in the context of the densities we used in our experiments, which reflect the highest abundance of spring concentrations in the field at Helgoland Roads (Greve et al. 2004) and should thus have represented the maximal expectable grazing impact.

T. longicornis is known to be a selective (Koski et al. 2005) and omnivorous grazer feeding on phytoplankton and microzooplankton (Kleppel et al. 1991; Kleppel 1993; Gentsch et al. 2009) of a wide size range in natural assemblages (O'Connors et al. 1980; Tackx et al. 1989, 1990). Furthermore, field populations of T. longicornis can have a substantial grazing impact on phytoplankton (Dam and Peterson 1993). At Helgoland Roads, the growth of phytoplankton in spring is followed by a drastic population increase of small calanoid copepods, while other mesozooplankton does not play a significant role (Greve et al. 2004). A new revision of long-term data on mesozooplankton (March/April 1975-2002) revealed that the group 'small calanoid copepods' represented $90 \%$ of the adult copepod community at the time of our experiments. Within the group, $T$. longicornis contributed up $60 \%$ to adult calanoid copepod numbers (mean 28\%, Kong, S.-M., unpublished). Given this fact, T. longicornis can be considered as an important copepod species in the North Sea planktonic food web in spring, and therefore, it was selected as representative copepod grazer in our experiments.

Low impacts on spring bloom development by copepods have been reported previously (Dagg et al. 1982; Dam et al. 1993), where the removal of daily primary production by copepods accounted for less than $25 \%$ of $P_{p}$. Although $T$. longicornis selected for microzooplankton in three out of four experiments, phytoplankton was always present in its diet $(18-65 \%)$. Furthermore, the maximum impact of copepods we detected in this study was $100 \%$, suggesting a structuring potential by copepods under certain conditions. Similar to the maximum values found by Dam and Peterson (1993), the average grazing impact of $T$. longicornis on the potential phytoplankton production we detected was $47 \%$ and therefore slightly higher than the $10-40 \%$ given by Calbet (2001) for copepods on a global scale. The range of per capita filtration rates of $T$. longicornis on phytoplankton we measured $\left(5-31 \mathrm{~mL} \mathrm{Ind.}{ }^{-1}\right.$ day $\left.^{-1}\right)$ is comparable to the values found by Dam (1986) in laboratory experiments (1-46 mL Ind. ${ }^{-1}$ day $\left.^{-1}\right)$. We found maximum filtration rates in our experiments at the phytoplankton bloom peak, which were around twice as high as reported for $T$. longicornis feeding on natural phytoplankton at comparable chlorophyll $a$ concentrations (Dam 1986). This difference may result from differences in phytoplankton assemblages but unfortunately information about the species composition is lacking in the study of Dam (1986). The highest ingestion rates of $T$. longicornis were also measured at the phytoplankton bloom peak. One copepod ingested $2.9 \mu \mathrm{gC}$ Ind. $^{-1}$ day $^{-1}$ in the form of phytoplankton, which is comparable to reported ingestion rates $(2.2 \mu \mathrm{gC}$ Ind. ${ }^{-1}$ day $^{-1}$ ) on diatom prey at similar chlorophyll $a$ concentrations (Dam 1986). In our experiment, we assumed all dinoflagellates, also mixotrophic species, to be heterotrophic. Consequently, we potentially underestimated copepod herbivory. However, the percentage of mixotrophic dinoflagellates in the microzooplankton community of the mesocosms was low $(0.2-3.1 \%$ of microzooplankton total carbon) and T. longicornis did not preferentially feed on them; thus, the underestimation of herbivory should have been low also.

Although the total carbon-specific ingestion rates (0.20-0.53, phytoplankton + microzooplankton prey) of T. longicornis were similar to results reported in previous studies (0.15-0.20: Daro 1985; 0.40-2.2: Koski et al. 2005), microzooplankton showed an almost sevenfold 
higher carbon-specific ingestion rate $\left(I_{c}\right)$ when preying on phytoplankton in contrast to the copepods. The total ingestion of microzooplankton we measured showed a mean of $53 \mu \mathrm{gC} \mathrm{L} \mathrm{day}^{-1}$, which is close to the mean value of $60 \mu \mathrm{gC} \mathrm{L}{ }^{1} \mathrm{day}^{-1}$ found during late winter and spring by Fonda Umani et al. (2005). They registered a mean impact of microzooplankton on potential phytoplankton production of $123 \%$. Similarly, in our experiments microzooplankton grazed on average $120 \%$ of the potential phytoplankton production. This is around twofold higher than results reported by Landry and Calbet (2004). They found an average grazing impact of 59-75\% of $P_{p}$ by microzooplankton across a spectrum of open ocean and coastal systems, whereas the lower border was found for estuarine systems with chlorophyll $a$ values similar to those of our experiment. In this study, the high availability of food during the bloom situation combined with a release from grazing pressure by metazoans allowed the development of a high microzooplankton grazer biomass in the mesocosms. Our results should therefore represent the maximum in microzooplankton grazing impact on phytoplankton in coastal regions under spring bloom conditions and has to be seen as the potential grazing impact of microzooplankton when topdown control by mesozooplankton is lacking.

Spring bloom dynamics in the North Sea around Helgoland are, of course, additionally subject to factors other than grazing (e.g., hydrography, salinity fluctuations, storm events and nutrient fluxes on a daily basis), which our mesocosm environment could not mimic. However, the close resemblance of chlorophyll $a$ development (and also composition of microzooplankton and phytoplankton assemblages, Löder et al., unpublished data) in our mesocosms to the natural situations (where mesograzers were present) suggests that microzooplankton drives the spring bloom dynamics of the phytoplankton community in waters around Helgoland (compare Fig. 1a).

\section{Optimal bloom exploitation through different feeding strategies of microzooplankton}

Different feeding strategies are recorded among heterotrophic dinoflagellates including direct engulfment, pallium- and peduncle- or tube feeding (Jacobson and Anderson 1986; Gaines and Elbrächter 1987). Ciliates are categorised as suspension, raptorial, deposit and diffusion feeders (Müller and Weisse 1994). Depending on the predators' feeding mode different prey is selected. Therefore, depending on the zooplankton community present at specific times of the year, feeding habits are directly mirrored by food selectivity patterns. Grazing selectivity itself also structures the phytoplankton composition (Irigoien et al.
2005). In our experiments, the microzooplankton community showed a large variety of food preferences and preferred food size spectra according to grazer species, grazer size and feeding mode.

Generally, dinoflagellates can feed on a wide range of prey (Jeong 1999) and are likely to be more quantitatively significant consumers of bloom-forming diatoms than copepods (Sherr and Sherr 2007). Athecate Gyrodinium spp. (20-120 $\mu \mathrm{m}$ length) and thecate Protoperidinium spp. (15-75 $\mu \mathrm{m}$ diameter) dominated the dinoflagellate grazer assemblage in our mesocosm study. Both genera are mainly associated with diatom blooms (Sherr and Sherr 2007). Dinoflagellates can feed and grow on variable predator to prey size ratios between 5.2:1 and 0.15:1 (Naustvoll 2000a, b). The upper limit of prey size reported by Naustvoll $(2000 a, b)$ is probably not reached by naked phagotrophs such as Gyrodinium spp. as they prefer food of their own size (Hansen 1992), but rather by thecate, pallium-feeding dinoflagellates like Protoperidinium spp. With regard to their size and feeding abilities, the present dinoflagellate taxa were able to feed on the biggest diatoms in the mesocosms.

Ciliates feed mainly on nanoplankton in an optimal size at approximately $1 / 10$ of their own size (Spittler 1973; Heinbokel 1978; Jonsson 1986). However, it is reported that they can feed on prey items sometimes larger than themselves (Smetacek 1981; Kahl 1932; Gifford 1985; Johansson et al. 2004). Ciliates are thus at times in direct feeding competition with copepods (Aberle et al. 2007) and dinoflagellates (Hansen 1992; Sherr and Sherr 2007). Strombidium capitatum, the dominating strombidiid is known to feed on small flagellates of different groups (Stoecker and Silver 1990; Crawford and Stoecker 1996). Other Strombidium and Strobilidium species as present in our experiment are considered to consume phytoplankton fractions ranging from 2 to $15 \mu \mathrm{m}$ (Christaki et al. 1998; Sime-Ngando et al. 1999; Aberle et al. 2007). Xu et al. (2005) found a big Cyclotrichium species, similar to the species present in the second half of the mesocosm bloom, feeding on different algae including diatoms. The main prey of ciliates in the mesocosm should, however, have been flagellates and smaller diatoms.

We found a highly diverse microzooplankton community during the spring bloom. Species of different size classes with different feeding modes were always present. It is therefore not surprising that microzooplankton grazed on all possible components of the phytoplankton ranging from smallest flagellates to large diatoms. Microzooplankton was even able to graze on very large bloomforming diatoms like Rhizosolenia spp. In our mesocosms we did not investigate factors such as cell death, cyst 
formation, sedimentation, parasitism or viral lysis that can lead to a decay of phytoplankton blooms. Senescence of cells and cell death as a consequence of nutrient depletion were, however, factors that certainly played an important role in our mesocosms, although one can argue about the remineralisation of nutrients due to microzooplankton grazing activity (Sherr and Sherr 2002). Nevertheless, the measured consumption of all available phytoplankton species should have been one of the most important factors for the decay of the bloom since it led to a strong suppression of phytoplankton and contributed to an almost complete decline within 3 weeks after the bloom peak.

Bloom stabilisation of less-favoured species due to selective grazing by microzooplankton

Phytoplankton blooms occur when external perturbations (improved light conditions in our study) promote improved growth conditions and open a loophole in the microbial loop (Irigoien et al. 2005). The species that are able to escape predation pressure by microzooplankton and have competitive advances compared to other phytoplankton (Flynn 2008) can benefit from improved growth conditions at the onset of a bloom thus enabling these species to form mass occurrences. As a consequence of rapidly dividing phytoplankton, the total exclusion from grazing is not required, only a difference in grazing rate resulting in a positive net growth. While phytoplankton competitors remain controlled by zooplankton grazing, the advance in net growth rate enables a particular phytoplankton species to form a bloom. Although microzooplankton is usually not able to prevent phytoplankton blooms under natural conditions (Sherr and Sherr 2009), its grazing can have at least a structuring influence and this circumstance has also been observed in previous studies (Riegman et al. 1993; Fonda Umani et al. 2005).

Irigoien et al. (2005) pointed out that among other factors, defence mechanisms (e.g. large cell sizes, colonies or spine formation) and selective predation of microzooplankton open a loophole for phytoplankton blooms of less edible, unfavoured species. As food selectivity is a constant process a pre-selection of phytoplankton species will have been already taken place in the field prior to the filling of our mesocosms. In early spring, total grazing rates of microzooplankton at the start of our experiment were not sufficiently high enough to suppress a spring bloom formation (see also Sherr and Sherr 2009). However, one can assume some directive influence of microzooplankton.

Flagellates made up one-third of the phytoplankton community at the start of the experiment. As they lost importance towards the end of March, we assume that the growth of flagellates was controlled by selective predation of microzooplankton thus hindering flagellates to form a bloom. By contrast, shortly after the onset of the bloom, the phytoplankton community in our mesocosms was dominated by three diatom genera: Rhizosolenia, Thalassiosira and Chaetoceros. Rhizosolenia was the largest diatom genus (mean length $288 \mu \mathrm{m}$ ) occurring in the mesocosms. Although Rhizosolenia was grazed to some extent, electivity values showed that it was less-preferred compared to other phytoplankton. It thereby had an advantage resulting in a relatively constant biomass value of $\sim 27 \%$ of the total phytoplankton biomass throughout the experiment. A good example for the opening of loopholes via selective grazing is the genus Thalassiosira. Two species occurred during the experiments, $T$. rotula and $T$. nordenskjoeldii, whereas the latter one dominated the total Thalassiosira spp. biomass with up to $92 \%$ and its dominance increased with the duration of the bloom. Both Thalassiosira species are able to form long chains but, in addition, T. nordenskjoeldii possesses spines. During our experiment, $T$. nordenskjoeldii was always a less-preferred prey item resulting in an increase in Thalassiosira spp. from 10 to $49 \%$ of total phytoplankton biomass. The other spine-possessing and chain-forming genus Chaetoceros showed a more ambivalent picture. It consisted of species of different size classes (10-40 $\mu \mathrm{m}$ diameter per cell). While the small Chaetoceros spp. $(10 \mu \mathrm{m})$ totally disappeared due to grazing, others, especially the bigger ones with long spines, were grazed less and remained as a constant fraction of the phytoplankton. Even if some bloom dynamics were observed, the consumption and selective grazing by microzooplankton stabilised the phytoplankton assemblage and widened the loophole for the bloom of three lessfavoured diatom taxa.

Microzooplankton species succession: a direct response to different food availability

The general succession pattern and species composition of the microzooplankton in the mesocosms was comparable to the patterns previously observed in the field at Helgoland Roads. In early spring, ciliates are the major group of microzooplankton grazers as they respond rapidly to increasing phytoplankton availability and dinoflagellates follow the ciliate bloom with some delay (Löder et al. 2011).

In the mesocosms, ciliates showed a direct response and distinct succession patterns in relation to food availability which was most pronounced in the genera Strombidium and Strobilidium. Their abundance was directly coupled with the availability of flagellate prey and resulted in a strong suppression of flagellates during the ciliate peak. With the disappearance of their predators due to food shortage the 
relaxation from grazing pressure re-enabled a positive net growth of flagellates. The fact that they did not disappear completely due to grazing is most probably due to selective predation on specific flagellate species. This predator-prey relationship was most obvious in Strombidium capitatum, which is known to be directly dependent on flagellate prey (Stoecker and Silver 1990) and forms cysts as soon as unfavourable conditions occur (Kim et al. 2008). Simultaneously with the disappearance of its potential prey (dinoflagellates $<15 \mu \mathrm{m}$ ) S. capitatum started to form cysts and its population collapsed within 1 week. After the decrease of strombidiids and strobilids, Cyclotrichium sp. a ciliate which also feeds on diatoms (Xu et al. 2005) started to dominate and towards the end of the bloom bacterivorous ciliates (Acineta sp., Euplotes sp.) gained in importance.

In contrast, dinoflagellates showed unclear succession patterns compared to ciliates. While two groups disappeared due to predation by ciliates (thecate and athecate dinoflagellates $<15 \mu \mathrm{m}$ ), the remaining dinoflagellate community remained relatively stable until the end of the experiment. This was most likely related to the fact that diatoms, the preferred prey of dinoflagellates (Sherr and Sherr 2007), were always present. The opposing patterns found for dinoflagellates and ciliates might also be related to a contrasting ecological strategy of these groups. Dinoflagellates are considered to have lower growth rates than ciliates (Hansen 1992), and therefore, their ability to react rapidly to enhanced food availability is limited. On the other hand, dinoflagellates can prey on almost every organic particle present in the oceans (Jeong 1999; Tillmann 2004). Additionally, they have a higher starving potential (Hansen 1992; Menden-Deuer et al. 2005), and thus can survive periods of food shortage (Sherr and Sherr 2007). In contrast, ciliates can respond rapidly to enhanced food availability showing growth rates higher than those of dinoflagellates (Strom and Morello 1998) but their potential to survive starvation periods is low (Jackson and Berger 1985; Hansen 1992) and they are more restricted to certain prey items (Tillmann 2004). Thus, the succession of microzooplankton observed in the present study is mainly triggered by the availability of food and contrasting survival strategies. In this context, ciliates can be considered specialists and dinoflagellates to be more generalists. Furthermore, the close resemblance of the mesocosm succession (no top-down control by mesozooplankton) with the general spring succession patterns as observed in the field (top-down control by mesozooplankton present) (Löder et al. 2011) suggest that microzooplankton species succession is mainly triggered by food availability (bottom-up), whereas abundance in the field should be subject to a combination of the factors food availability and predation (combined effects of bottom-up and top-down control).

Potential factors determining the microzooplankton bloom in the mesocosms

Interestingly, microzooplankton biomass started to decline at a mean rate of $-0.15 \mathrm{day}^{-1}$ to a level close to the start value immediately after it reached its peak. The collapse started 1 week after the phytoplankton bloom peak, even though a considerable amount of phytoplankton was still available. Furthermore, neither phytoplankton composition nor grazing impact or food selectivity of microzooplankton changed greatly. However, all microzooplankton taxa, except Strombidium spp. and rotifers (Table 3), displayed mortality rates of -0.05 to $-0.52 \mathrm{day}^{-1}$.

Besides strong predator-prey relations that lead to the death of the predator due to starvation when its prey is absent (Jackson and Berger 1985; this study) changes in the food quality of the preferred food might be a plausible explanation for the decline in microzooplankton biomass. This seems even more reasonable given the fact that prey availability was not limited. With the duration of the bloom in our mesocosms phytoplankton got increasingly nutrientdepleted (Schoo 2010), and therefore, it seems likely that phytoplankton during the post-bloom phase did not meet the nutritional needs of the microzooplankton. The effort to capture, handle, digest the prey and egest the excess carbon might have been more energy demanding than the energy benefit the prey offered resulting in low growth or even mortality.

Negative effects due to poor food have been reported (Jensen and Hessen 2007) and if predators have the choice between good and bad food they naturally choose the good one. Other microzooplankters, which feed on nutrientlimited phytoplankton, represent the better food when compared to the phytoplankton itself (Malzahn et al. 2010). Thus, an additional effect introduced by "bad quality phytoplankton" may have been predation within microzooplankton. Pronounced carnivory towards the end of phytoplankton blooms has been described by Irigoien et al. (2005) and in our experiment microzooplankton might also have switched its feeding strategy.

Towards the end of the bloom rotifers gained in importance (up to $28 \%$ of biomass). They are the only group, besides Strombidium spp. (unimportant from a biomass perspective) which showed positive growth rates. About $10-40 \%$ of rotifer food can consist of heterotrophic components as rotifers are efficient predators on protozoans (Arndt 1993). It is therefore most likely that the combined effect of both, predation within the microzooplankton especially by rotifers and the bad nutritional quality of the 
food sources, resulted in an overall decline in microzooplankton abundance.

Copepod selective feeding and the microzooplankton fate in a real bloom

Microzooplankton is able to compete with copepods for the same food and to exploit food stocks more efficiently due to their fast metabolic abilities and growth rates (Hansen 1992; Sherr and Sherr 2007; Aberle et al. 2007). They in turn are preferred food for higher trophic levels, e.g. mesozooplankton, even if phytoplankton is available in high numbers but with a low food quality (Hansen et al. 1993). Although the degree of herbivory differs between copepod species (Maar et al. 2004), microzooplankton contributes as a substantial part to copepods' diets and it is often positively selected (Nejstgaard et al. 1997; Fileman et al. 2007). Gifford and Dagg (1988) showed for the copepod Acartia tonsa that even when microzooplankton was present in low concentrations (3\% of total carbon) it made up to $41 \%$ of the copepods diet. Grazing on microzooplankton by copepods can result severe trophic cascade effects. The release of microzooplankton grazing pressure can promote nanoflagellates, an important prey of ciliates, and thus affect bacterial abundance negatively as bacteria are the main food source of nanoflagellates (Zöllner et al. 2009). Even more pronounced effects were reported for chlorophyll $a$ concentrations: copepod grazers reduced microzooplankton biomass and led to overall higher chlorophyll $a$ concentrations due to the release of small sized flagellates from microzooplankton grazing (Sommer et al. 2003, 2005a, b).

Trophic upgrading of food by heterotrophic protists (Martin-Creuzburg et al. 2005; Tang and Taal 2005; Bec et al. 2006) has been demonstrated. Data also show that protozoan grazers can compensate stoichiometric imbalances to a certain extent when they feed on low quality food (Malzahn et al. 2010). This fact, as well as their capacity to synthesise highly unsaturated fatty acids and sterols makes them good quality food from a copepod perspective (Klein Breteler et al. 1999; Tang and Taal 2005). With decreasing phytoplankton food quality in our experiments $T$. longicornis changed its diet gradually from phytoplankton-dominated to microzooplanktondominated over the course of the bloom. Hence, we observed high positive selection for microzooplankton species, especially larger dinoflagellates and ciliates. This is in contrast to a laboratory study by Jakobsen et al. (2005) where T. longicornis showed no selectivity when offered mixed prey including a ciliate. Koski et al. (2005) found only a weak selectivity in $T$. longicornis during a mesocosm Phaeocystis globosa bloom. However, studies cited in Koski et al. (2005) provided evidence for a selective feeding capability of $T$. longicornis, especially a positive selection for microzooplankton, dinoflagellates and ciliates. Given the fact that copepod species like T. longicornis positively select for microzooplankton and can have a severe grazing impact on microzooplankton we assume that microzooplankton biomass can be topdown controlled by copepod grazing during spring bloom situations at Helgoland Roads if the density of predators is high enough.

\section{Conclusions}

1. Microzooplankton reacted quickly to enhanced prey availability and its high grazing rates contributed to a decrease to pre-bloom concentrations of phytoplankton.

2. Microzooplankton was the more efficient phytoplankton grazer when compared with copepods.

3. Selective grazing by microzooplankton supported a bloom of less-favoured phytoplankton species and stabilised the shares of bloom-forming species during the course of the bloom.

4. Ciliates responded with rapid growth and mortality to differences in prey availability, leading to a short but large peak. Dinoflagellates had a broader food spectrum and lower growth and mortality rates, which led to a longer duration of the dinoflagellate bloom.

5. As a substantial part of the copepods' diet microzooplankton became more important with decreasing food quality of the phytoplankton during the course of the bloom.

Acknowledgments This study was part of a $\mathrm{PhD}$ thesis within the Food Web Project at the Alfred Wegener Institute for Polar and Marine Research and we are grateful for the funding. Special thanks to Prof. Ulrich Sommer, Thomas Hansen and Sebastian Meyer at the IFM-GEOMAR (Kiel) for providing us the "Copacabana" light control programme and who helped us in words and deeds. Many thanks to the technical department of the BAH for all the perfect "short notice" solutions and to Arne Malzahn for his technical support. Special thanks also to the "Copepod Hunter" Katherina Schoo for catching and sorting out all the copepods for our experiments. Furthermore, thanks to the crews of the research vessels Uthörn and Aade, Kristine Carstens, Silvia Peters and the whole team of the AWI Food Web Project. Last but not least many thanks for the comments of three anonymous reviewers which helped us a lot for improving this manuscript.

\section{Appendix}

See Tables 5, 6, 7, and 8 . 
Table 5 Microzooplankton grazing $g\left(\right.$ day $\left.^{-1}\right)$, phytoplankton growth rates $k\left(\right.$ day $\left.^{-1}\right)$ and instantaneous growth rate values $\mu_{o}\left(\right.$ day $\left.{ }^{-1}\right)$ from bottles without added nutrients, percentage of initial stock $P_{i}(\%)$ and potential production $P_{p}(\%)$ grazed as determined in four dilution experiments for each registered prey taxon

\begin{tabular}{|c|c|c|c|c|c|c|c|c|c|c|c|c|c|c|}
\hline \multirow[t]{3}{*}{ Phytoplankton } & \multicolumn{14}{|c|}{ Microzooplankton } \\
\hline & \multicolumn{7}{|c|}{ Experiment 1 (MMC $30.33 \mu \mathrm{gC} \mathrm{L}^{-1}$ ) } & \multicolumn{7}{|c|}{ Experiment $2\left(\mathrm{MMC} 74.47 \mu \mathrm{gC} \mathrm{L} \mathrm{L}^{-1}\right)$} \\
\hline & $k$ & $P$ & $g$ & $P$ & $\mu_{0}$ & $P_{i}$ & $P_{p}$ & $k$ & $P$ & $g$ & $P$ & $\mu_{0}$ & $P_{i}$ & $P_{p}$ \\
\hline Pseudonitzschia spp. $(120 \mu \mathrm{m})$ & 0.43 & $* * *$ & 0.62 & $* *$ & 0.31 & 86 & 172 & 0.87 & $* * * *$ & 1.04 & $* * * *$ & 1.52 & 183 & \\
\hline Pseudonitzschia spp. $(80 \mu \mathrm{m})$ & 0.14 & & 0.29 & & -0.46 & 34 & 0 & 0.57 & $* * *$ & 0.66 & * & 0.85 & 93 & \\
\hline Pseudonitzschia spp. $(60 \mu \mathrm{m})$ & 0.44 & & 1.08 & & 0.51 & 195 & 164 & 0.99 & $* * * *$ & 0.88 & $*$ & 0.50 & 142 & 1 \\
\hline Navicula spp. $(40 \mu \mathrm{m})$ & 0.66 & $*$ & 0.39 & $*$ & 0.16 & 48 & 220 & 0.98 & $* * * *$ & 1.35 & $* * * *$ & 1.62 & 285 & \\
\hline Navicula spp. $(20 \mu \mathrm{m})$ & 0.08 & & -0.21 & & -0.28 & 0 & 74 & 0.20 & & 0.52 & $*$ & 0.23 & 68 & 19 \\
\hline Navicula spp. $(10 \mu \mathrm{m})$ & -0.32 & $*$ & -0.50 & & -0.39 & 0 & 135 & 0.24 & $*$ & 0.44 & $*$ & 0.16 & 56 & 23 \\
\hline Chaetoceros danicus & 1.14 & $* *$ & 1.11 & $* * * *$ & 0.65 & 203 & 141 & 0.40 & $* *$ & 0.16 & & 0.30 & 18 & 5 \\
\hline Chaetoceros spp. $(40 \mu \mathrm{m})$ & 0.72 & $* *$ & 1.16 & $* *$ & 0.62 & 220 & 149 & 1.07 & $* * * *$ & 0.91 & $* *$ & 1.25 & 150 & 8 \\
\hline Chaetoceros spp. $(30 \mu \mathrm{m})$ & 0.48 & $* *$ & 0.77 & $* *$ & 0.86 & 116 & 93 & 0.59 & $* * *$ & 0.54 & $*$ & 0.93 & 71 & 6 \\
\hline Chaetoceros spp. $(20 \mu \mathrm{m})$ & 0.41 & & 0.55 & $*$ & 0.40 & 72 & 129 & 1.60 & $* * * *$ & 1.69 & $* * * *$ & 1.70 & 443 & 100 \\
\hline Chaetoceros spp. $(10 \mu \mathrm{m})$ & 0.71 & $*$ & 1.19 & $*$ & 0.73 & 230 & 135 & 1.34 & $* * *$ & 0.75 & & 0.66 & 113 & 109 \\
\hline Rhizosolenia styliformis/hebetata & 0.32 & & 0.35 & $* *$ & 0.03 & 42 & 1063 & & $* * * *$ & 0.61 & $* * * *$ & 0.62 & 85 & \\
\hline Rhizosolenia pungens/setigera & -0.58 & $*$ & -0.73 & $*$ & 0.05 & 0 & 0 & 1.09 & $* * * *$ & 1.02 & $* * * *$ & 1.03 & 177 & 9 \\
\hline Thalassiosira nordenskioeldii & 0.50 & $* * *$ & 0.50 & * & 0.33 & 65 & 141 & 0.66 & $* * * *$ & 0.39 & $*$ & 0.63 & 47 & 6 \\
\hline Thalassiosira rotula & 0.56 & $* * * *$ & 0.68 & $* *$ & 0.64 & 96 & 104 & -0.14 & & -0.29 & & 0.16 & 0 & \\
\hline Flagellates $(25 \mu \mathrm{m})$ & 0.54 & & 0.68 & $*$ & 0.83 & 98 & 88 & 0.49 & $*$ & 0.74 & $*$ & 0.78 & 110 & 9 \\
\hline Flagellates $(20 \mu \mathrm{m})$ & 0.11 & & 0.02 & & -0.12 & 2 & 0 & 0.07 & & 0.47 & $*$ & -0.06 & 60 & \\
\hline Flagellates $(15 \mu \mathrm{m})$ & -0.52 & $* *$ & -0.36 & & -0.47 & 0 & 0 & 0.53 & $* * *$ & 0.91 & $* * *$ & 0.69 & 149 & 119 \\
\hline Flagellates $(10 \mu \mathrm{m})$ & 0.49 & $*$ & 0.31 & $* *$ & -0.23 & 36 & 0 & 0.46 & $* *$ & 0.66 & $* *$ & 0.58 & 94 & 109 \\
\hline Flagellates $(5 \mu \mathrm{m})$ & 0.24 & & 0.28 & $* *$ & -0.61 & 32 & 0 & 0.79 & $* * * *$ & 1.21 & $* * *$ & 1.42 & 236 & 9 \\
\hline \multirow[t]{2}{*}{ Total phytoplankton } & 0.41 & $*$ & 0.43 & $*$ & 0.17 & 53 & 223 & 0.80 & $* * * *$ & 0.66 & $* * *$ & 0.77 & 93 & 9 \\
\hline & \multicolumn{7}{|c|}{ Experiment 3 (MMC $93.82 \mu \mathrm{gC} \mathrm{L}^{-1}$ ) } & \multicolumn{7}{|c|}{ Experiment 4 (MMC $33.12 \mu \mathrm{gC} \mathrm{L}^{-1}$ ) } \\
\hline Pseudonitzschia spp. $(120 \mu \mathrm{m})$ & 0.38 & $* * *$ & 0.41 & $*$ & 0.46 & 51 & 91 & 0.78 & $* * * *$ & 0.44 & $* * * *$ & 0.75 & 55 & 6 \\
\hline Pseudonitzschia spp. $(80 \mu \mathrm{m})$ & 0.91 & $* * * *$ & 0.94 & $* * *$ & 1.26 & 156 & 85 & 1.08 & $* * * *$ & 1.14 & $* * *$ & 1.82 & 213 & 8 \\
\hline Pseudonitzschia spp. $(60 \mu \mathrm{m})$ & 1.63 & $* * * *$ & 2.04 & $* * * *$ & 2.02 & 666 & 100 & 1.02 & $* * *$ & 1.22 & $* *$ & 1.46 & 239 & 9 \\
\hline Navicula spp. $(40 \mu \mathrm{m})$ & 1.35 & $* * * *$ & 1.62 & $* * * *$ & 0.16 & 404 & 541 & 0.20 & & 0.50 & $*$ & -0.20 & 66 & \\
\hline Navicula spp. $(20 \mu \mathrm{m})$ & 0.15 & & 0.52 & & 0.45 & 68 & 111 & -0.18 & & 0.18 & & 0.18 & 19 & 9 \\
\hline Navicula spp. $(10 \mu \mathrm{m})$ & 0.30 & & 0.62 & $*$ & 0.23 & 85 & 223 & 1.32 & $* *$ & 1.43 & $*$ & 2.18 & 316 & \\
\hline Chaetoceros danicus & -0.03 & & -0.07 & & -0.02 & 0 & 0 & -0.28 & & -0.11 & & 0.21 & 0 & \\
\hline Chaetoceros spp. $(40 \mu \mathrm{m})$ & 1.09 & $* * * *$ & 1.42 & $* * *$ & 1.72 & 313 & 92 & 1.18 & $* * * *$ & 1.00 & $* *$ & 1.67 & 173 & 78 \\
\hline Chaetoceros spp. $(30 \mu \mathrm{m})$ & 0.45 & $*$ & 0.34 & & 0.50 & 40 & 73 & 1.17 & $* * * *$ & 0.84 & $* * * *$ & 1.66 & 131 & 7 \\
\hline Chaetoceros spp. $(20 \mu \mathrm{m})$ & 0.27 & $*$ & 0.51 & $*$ & 0.08 & 66 & 515 & 0.64 & $* * * *$ & 0.16 & & 0.68 & 18 & 31 \\
\hline Chaetoceros spp. $(10 \mu \mathrm{m})$ & - & - & - & - & - & - & - & - & - & - & - & - & - & (s) \\
\hline Rhizosolenia styliformis/hebetata & 0.41 & $* * * *$ & 0.47 & $* * * *$ & 0.73 & 61 & 73 & 0.50 & $* * * *$ & 0.36 & $* *$ & 0.65 & 43 & 63 \\
\hline Rhizosolenia pungens/setigera & 0.64 & $* *$ & 0.66 & $*$ & 0.74 & 93 & 92 & 0.33 & & 0.28 & & 0.06 & 32 & 409 \\
\hline Thalassiosira nordenskioeldii & 0.17 & $* *$ & 0.49 & $* * * *$ & 0.49 & 63 & 99 & 0.62 & $* * * *$ & 0.58 & $* * * *$ & 0.73 & 79 & 85 \\
\hline Thalassiosira rotula & 0.51 & $* * *$ & 0.87 & ** & 1.14 & 138 & 85 & 0.86 & $* * * *$ & 0.70 & $* * *$ & 1.20 & 102 & 72 \\
\hline Flagellates $(25 \mu \mathrm{m})$ & 1.34 & $*$ & 1.92 & $*$ & 2.36 & 584 & 94 & 1.33 & & 1.33 & & 3.04 & 278 & 77 \\
\hline Flagellates $(20 \mu \mathrm{m})$ & 0.43 & & 1.03 & $*$ & 0.76 & 180 & 121 & 0.66 & $*$ & 0.98 & $*$ & 0.73 & 165 & 121 \\
\hline Flagellates $(15 \mu \mathrm{m})$ & 0.84 & $* * * *$ & 1.38 & $* * * *$ & 0.72 & 299 & 146 & 0.28 & & 0.50 & & 0.68 & 65 & 7 \\
\hline Flagellates $(10 \mu \mathrm{m})$ & 0.82 & $* * * *$ & 0.99 & $* * * *$ & 0.95 & 169 & 102 & -0.49 & & -0.12 & & -0.59 & 0 & \\
\hline Flagellates $(5 \mu \mathrm{m})$ & 0.57 & $* * * *$ & 0.54 & $* *$ & 0.76 & 72 & 79 & 0.007 & & 0.26 & $*$ & -0.33 & 30 & \\
\hline Total phytoplankton & 0.39 & $* * * *$ & 0.54 & $* * * *$ & 0.65 & 72 & 87 & 0.41 & $* * * *$ & 0.39 & $* * *$ & 0.52 & 48 & 80 \\
\hline
\end{tabular}

Food saturation given in italic values; negative $P_{i}$ and $P_{p}$ values resulting from negative $g\left(P_{i}\right)$ or $\mu_{o}\left(P_{p}\right)$ were set to zero, also positive $P_{p}$ values resulting from negative $g$ and $\mu_{o}$

$M M C$ mean microzooplankton carbon biomass

$P$ values from linear regression analysis of apparent phytoplankton growth against dilution factor $(n=36)$, $P<0.05 ; * * P<0.01$; *** $P<0.001$; **** $P<0.0001$ 
Table 6 Microzooplankton carbon-specific filtration rates $F_{c}\left(\mathrm{~mL} \mu \mathrm{gC}\right.$ predator ${ }^{-1}$ day $\left.^{-1}\right)$ and carbon-specific ingestion rates $I_{c}(\mu \mathrm{gC}$ prey $\mu \mathrm{gC}$ predator $^{-1}$ day $\left.^{-1}\right)$, total ingestion rates of the microzooplankton community $I_{\text {total }}\left(\mu \mathrm{gC}\right.$ prey $\mathrm{L}^{-1}$ day $\left.^{-1}\right)$ and electivity $E^{*}(-)$ for each registered prey taxon

\begin{tabular}{|c|c|c|c|c|c|c|c|c|c|c|}
\hline \multirow[t]{3}{*}{ Phytoplankton } & \multicolumn{10}{|c|}{ Microzooplankton } \\
\hline & \multicolumn{5}{|c|}{ Experiment 1 (MMC $\left.30.33 \mu \mathrm{gC} \mathrm{L}^{-1}\right)$} & \multicolumn{5}{|c|}{ Experiment $2\left(\mathrm{MMC} 74.47 \mu \mathrm{gC} \mathrm{L}{ }^{-1}\right)$} \\
\hline & $F_{c}$ & $P$ & $I_{c}$ & $P$ & $E^{*}$ & $F_{c}$ & $P$ & $I_{c}$ & $P$ & $E^{*}$ \\
\hline Pseudonitzschia spp. $(120 \mu \mathrm{m})$ & 20.52 & $* *$ & 0.0179 & $* *$ & 0.11 & 13.97 & $* * * *$ & 0.0070 & $* * * *$ & 0.16 \\
\hline Pseudonitzschia spp. $(80 \mu \mathrm{m})$ & 9.58 & & 0.0015 & & -0.26 & 8.83 & $*$ & 0.0009 & * & -0.06 \\
\hline Pseudonitzschia spp. $(60 \mu \mathrm{m})$ & 35.71 & & 0.0003 & & 0.37 & 11.86 & $*$ & 0.0002 & * & 0.08 \\
\hline Navicula spp. $(40 \mu \mathrm{m})$ & 12.94 & * & 0.0008 & $*$ & -0.12 & 18.10 & $* * * *$ & 0.0018 & $* * * *$ & 0.29 \\
\hline Navicula spp. $(20 \mu \mathrm{m})$ & -6.98 & & -0.0034 & & -1.00 & 7.00 & $*$ & 0.0032 & * & -0.18 \\
\hline Navicula spp. $(10 \mu \mathrm{m})$ & -16.40 & & -0.0542 & & -1.00 & 5.97 & $*$ & 0.0244 & $*$ & -0.26 \\
\hline Chaetoceros danicus & 36.51 & $* * * *$ & 0.0914 & $* * * *$ & 0.38 & 2.20 & & 0.0046 & & -0.64 \\
\hline Chaetoceros spp. $(40 \mu \mathrm{m})$ & 38.31 & $* *$ & 0.0301 & $* *$ & 0.40 & 12.28 & $* *$ & 0.0096 & $* *$ & 0.10 \\
\hline Chaetoceros spp. $(30 \mu \mathrm{m})$ & 25.33 & $* *$ & 0.0500 & $* *$ & 0.21 & 7.23 & $*$ & 0.0292 & $*$ & -0.16 \\
\hline Chaetoceros spp. $(20 \mu \mathrm{m})$ & 17.97 & $*$ & 0.1322 & * & 0.04 & 22.72 & $* * * *$ & 0.0673 & $* * * *$ & 0.39 \\
\hline Chaetoceros spp. $(10 \mu \mathrm{m})$ & 39.33 & $*$ & 0.0826 & $*$ & 0.41 & 10.12 & & 0.0046 & & 0.00 \\
\hline Rhizosolenia styliformis/hebetata & 11.66 & $* *$ & 0.4002 & $* *$ & -0.17 & 8.23 & $* * * *$ & 0.2548 & $* * * *$ & -0.10 \\
\hline Rhizosolenia pungens/setigera & -24.13 & $*$ & -0.0588 & $*$ & -1.00 & 13.67 & $* * * *$ & 0.1286 & $* * * *$ & 0.15 \\
\hline Thalassiosira nordenskioeldii & 16.48 & $*$ & 0.5005 & $*$ & 0.00 & 5.21 & $*$ & 0.1975 & $*$ & -0.32 \\
\hline Thalassiosira rotula & 22.27 & $* *$ & 0.2031 & $* *$ & 0.15 & -3.95 & & -0.0396 & & -1 \\
\hline Flagellates $(25 \mu \mathrm{m})$ & 22.57 & $*$ & 0.0073 & $*$ & 0.16 & 9.99 & $*$ & 0.0035 & $*$ & 0.00 \\
\hline Flagellates $(20 \mu \mathrm{m})$ & 0.73 & & 0.0006 & & -0.91 & 6.32 & $*$ & 0.0092 & $*$ & -0.23 \\
\hline Flagellates $(15 \mu \mathrm{m})$ & -11.72 & & -0.0262 & & -1.00 & 12.23 & $* * *$ & 0.0263 & $* * *$ & 0.10 \\
\hline Flagellates $(10 \mu \mathrm{m})$ & 10.17 & $* *$ & 0.0524 & $* *$ & -0.24 & 8.89 & $* *$ & 0.0271 & $* *$ & -0.06 \\
\hline Flagellates $(5 \mu \mathrm{m})$ & 9.17 & $* *$ & 0.0698 & $* *$ & -0.28 & 16.26 & $* * *$ & 0.0366 & $* * *$ & 0.24 \\
\hline Total phytoplankton & 14.02 & $*$ & 1.57 & $*$ & & 8.85 & $* * *$ & 1.00 & $* * *$ & \\
\hline \multirow[t]{2}{*}{ Related to total microzooplankton $\mathrm{C} \mathrm{L}^{-1}$} & & $I_{\text {total }}$ & 47.65 & & & & $I_{\text {total }}$ & 74.58 & & \\
\hline & \multicolumn{5}{|c|}{ Experiment 3 (MMC $93.82 \mu \mathrm{gC} \mathrm{L} \mathrm{L}^{-1}$ ) } & \multicolumn{5}{|c|}{ Experiment 4 (MMC $33.12 \mu \mathrm{gC} \mathrm{L} \mathrm{L}^{-1}$ ) } \\
\hline Pseudonitzschia spp. $(120 \mu \mathrm{m})$ & 4.41 & $*$ & 0.0043 & * & -0.36 & 13.23 & $* * * *$ & 0.0105 & $* * * *$ & -0.18 \\
\hline Pseudonitzschia spp. $(80 \mu \mathrm{m})$ & 10.01 & $* * *$ & 0.0008 & $* * *$ & 0.03 & 34.47 & $* * *$ & 0.0021 & $* * *$ & 0.29 \\
\hline Pseudonitzschia spp. $(60 \mu \mathrm{m})$ & 21.70 & $* * * *$ & 0.0002 & $* * * *$ & 0.40 & 36.88 & $* *$ & 0.0002 & $* *$ & 0.32 \\
\hline Navicula spp. $(40 \mu \mathrm{m})$ & 17.24 & $* * * *$ & 0.0008 & $* * * *$ & 0.29 & 15.21 & $*$ & 0.0007 & $*$ & -0.11 \\
\hline Navicula spp. $(20 \mu \mathrm{m})$ & 5.52 & & 0.0012 & & -0.26 & 5.36 & & 0.0007 & & -0.56 \\
\hline Navicula spp. $(10 \mu \mathrm{m})$ & 6.56 & $*$ & 0.0026 & $*$ & -0.18 & 43.05 & $*$ & 0.0019 & $*$ & 0.39 \\
\hline Chaetoceros danicus & -0.78 & & -0.0016 & & -1 & -3.34 & & -0.0041 & & -1 \\
\hline Chaetoceros spp. $(40 \mu \mathrm{m})$ & 15.12 & $* * *$ & 0.0117 & $* * *$ & 0.23 & 30.30 & $* *$ & 0.0146 & $* *$ & 0.23 \\
\hline Chaetoceros spp. $(30 \mu \mathrm{m})$ & 3.59 & & 0.0185 & & -0.45 & 25.33 & $* * * *$ & 0.0511 & $* * * *$ & 0.14 \\
\hline Chaetoceros spp. $(20 \mu \mathrm{m})$ & 5.41 & $*$ & 0.0336 & $*$ & -0.27 & 4.94 & & 0.0275 & & -0.59 \\
\hline Chaetoceros spp. $(10 \mu \mathrm{m})$ & - & - & - & - & - & - & - & - & - & - \\
\hline Rhizosolenia styliformis/hebetata & 5.05 & $* * * *$ & 0.1467 & $* * * *$ & -0.30 & 10.75 & $* *$ & 0.2776 & $* *$ & -0.28 \\
\hline Rhizosolenia pungens/setigera & 7.02 & $*$ & 0.0321 & $*$ & -0.14 & 8.46 & & 0.0192 & & -0.38 \\
\hline Thalassiosira nordenskioeldii & 5.19 & $* * * *$ & 0.1874 & $* * * *$ & -0.29 & 17.65 & $* * * *$ & 0.3726 & $* * * *$ & -0.03 \\
\hline Thalassiosira rotula & 9.24 & $* *$ & 0.0501 & $* *$ & -0.01 & 21.19 & $* * *$ & 0.0669 & $* * *$ & 0.06 \\
\hline Flagellates $(25 \mu \mathrm{m})$ & 20.49 & $*$ & 0.0013 & $*$ & 0.37 & 40.18 & & 0.0017 & & 0.36 \\
\hline Flagellates $(20 \mu \mathrm{m})$ & 10.97 & $*$ & 0.0017 & $*$ & 0.08 & 29.47 & $*$ & 0.0049 & $*$ & 0.22 \\
\hline Flagellates $(15 \mu \mathrm{m})$ & 14.75 & $* * * *$ & 0.0020 & $* * * *$ & 0.22 & 15.09 & & 0.0027 & & -0.11 \\
\hline Flagellates $(10 \mu \mathrm{m})$ & 10.54 & $* * * *$ & 0.0151 & $* * * *$ & 0.06 & -3.70 & & -0.0284 & & -1 \\
\hline Flagellates $(5 \mu \mathrm{m})$ & 5.80 & $* *$ & 0.0834 & $* *$ & -0.24 & 7.96 & $*$ & 0.0857 & * & -0.41 \\
\hline Total phytoplankton & 5.77 & $* * * *$ & 0.62 & $* * * *$ & & 11.89 & $* * *$ & 0.97 & $* * *$ & \\
\hline Related to total microzooplankton $\mathrm{C} \mathrm{L}^{-1}$ & & $I_{\text {total }}$ & 58.02 & & & & $I_{\text {total }}$ & 32.13 & & \\
\hline
\end{tabular}

Positive selection given in italic values

$M M C$ mean microzooplankton carbon biomass

$P$ values are the same as for the grazing rates of microzooplankton, $* P<0.05 ; * * P<0.01 ; * * * P<0.001 ; * * * * P<0.0001$ 
Table 7 Temora longicornis grazing $g\left(\right.$ day $\left.^{-1}\right)$, phytoplankton and microzooplankton growth rates $k\left(\right.$ day $\left.^{-1}\right)$ and phytoplankton instantaneous growth rate values $\mu_{o}\left(\mathrm{day}^{-1}\right)$ from bottles without added nutrients, percentage of initial stock $P_{i}(\%)$ and potential production $P_{p}(\%)$ grazed as determined in four grazing experiments for each registered prey taxon

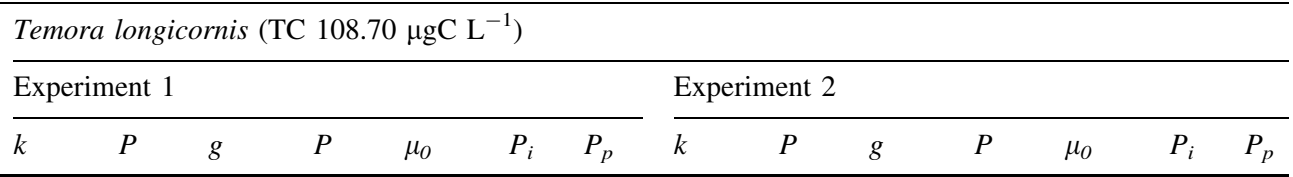

\section{Phytoplankton}

Pseudonitzschia spp. $(120 \mu \mathrm{m})$

Pseudonitzschia spp. $(80 \mu \mathrm{m})$

Pseudonitzschia spp. $(60 \mu \mathrm{m})$

Navicula spp. (40 $\mu \mathrm{m})$

Navicula spp. $(20 \mu \mathrm{m})$

Navicula spp. $(10 \mu \mathrm{m})$

Chaetoceros danicus

Chaetoceros spp. $(40 \mu \mathrm{m})$

Chaetoceros spp. $(30 \mu \mathrm{m})$

Chaetoceros spp. $(20 \mu \mathrm{m})$

Chaetoceros spp. $(10 \mu \mathrm{m})$

Rhizosolenia styliformis/hebetata

Rhizosolenia pungens/setigera

Thalassiosira nordenskioeldii

Thalassiosira rotula

Flagellates $(25 \mu \mathrm{m})$

Flagellates $(20 \mu \mathrm{m})$

Flagellates $(15 \mu \mathrm{m})$

Flagellates $(10 \mu \mathrm{m})$

Flagellates $(5 \mu \mathrm{m})$

Total phytoplankton

\section{Microzooplankton}

Gyrodinium spp. (30-75 $\mu \mathrm{m})$

Gyrodinium spp. (75-120 $\mu \mathrm{m})$

Protoperidinium spp. $(20-40 \mu \mathrm{m})$

Protoperidinium spp. $(50-80 \mu \mathrm{m})$

Ceratium spp.

Torodinium spp.

Other athecate dinoflagellates

Other thecate dinoflagellates

Strombidium spp. $(25-40 \mu \mathrm{m})$

Strombidium spp. $(40-110 \mu \mathrm{m})$

Strobilidium spp.

Myrionecta rubra

Other Cyclotrichids

Haptorids

Tintinnids

Other Ciliates

Thecate amoeba $\mathrm{sp}$.

Rotifers

Total microzooplankton

\begin{tabular}{|c|c|c|c|}
\hline-0.11 & & -0.02 & \\
\hline-0.25 & & 0.07 & \\
\hline-0.29 & & 0.51 & \\
\hline 0.25 & & 0.35 & \\
\hline 0.30 & $*$ & 0.01 & \\
\hline 0.15 & & -0.06 & \\
\hline 0.00 & & 1.01 & $* *$ \\
\hline-0.30 & & 0.17 & \\
\hline-0.15 & & 0.29 & \\
\hline-0.13 & & 0.41 & $* *$ \\
\hline-0.31 & & -0.10 & \\
\hline-0.04 & & 0.13 & \\
\hline 0.17 & & 0.29 & \\
\hline 0.02 & & 0.17 & $*$ \\
\hline-0.07 & & 0.30 & $* *$ \\
\hline 0.03 & & 0.99 & \\
\hline-0.05 & & 0.32 & \\
\hline-0.24 & & 0.02 & \\
\hline 0.14 & & 0.31 & \\
\hline-0.04 & & -0.14 & \\
\hline-0.01 & & 0.17 & $* * *$ \\
\hline-0.05 & & 1.12 & $* *$ \\
\hline 0.30 & $*$ & 0.95 & $* *$ \\
\hline 0.41 & & -0.14 & \\
\hline 0.17 & & 1.15 & $* *$ \\
\hline-0.21 & & 1.51 & \\
\hline 0.02 & & 0.23 & \\
\hline 0.02 & & 0.18 & \\
\hline 0.40 & $* * * *$ & -0.03 & \\
\hline-0.12 & & 0.39 & \\
\hline 0.32 & $*$ & 1.24 & $* * *$ \\
\hline 0.26 & & 0.92 & $*$ \\
\hline 0.18 & & 0.95 & $* *$ \\
\hline 0.00 & & 0.00 & \\
\hline-0.28 & & 0.93 & $*$ \\
\hline 0.11 & & -0.08 & \\
\hline- & & - & \\
\hline 0.16 & & 0.32 & $* * *$ \\
\hline 0.00 & & - & \\
\hline 0.21 & $* * * *$ & 0.40 & $* * * *$ \\
\hline
\end{tabular}

$\begin{array}{rrrrl}0.31 & 0 & 0 & -0.15 & * \\ -0.46 & 8 & 0 & -0.08 & \\ 0.51 & 67 & 99 & 0.26 & \\ 0.16 & 42 & 202 & -0.27 & \\ -0.28 & 1 & 0 & -0.25 & \\ -0.39 & 0 & 0 & -0.16 & \\ 0.65 & 174 & 134 & 0.36 & \\ 0.62 & 19 & 34 & 0.33 & \\ 0.86 & 33 & 43 & 0.31 & \\ 0.40 & 50 & 102 & 0.08 & \\ 0.73 & 0 & 0 & 1.29 & \\ 0.03 & 14 & 432 & -0.06 & \\ 0.05 & 34 & 500 & 0.20 & \\ 0.33 & 18 & 55 & 0.34 & * * \\ 0.64 & 35 & 55 & 0.15 & \\ 0.83 & 170 & 112 & -0.20 & \\ -0.12 & 38 & 0 & -0.36 & \\ -0.47 & 2 & 0 & -0.38 & * * \\ -0.23 & 37 & 0 & -0.09 & \\ -0.61 & 0 & 0 & -0.44 & \\ 0.17 & 18 & 100 & 0.13 & \end{array}$

0.02

$-0.04$

$-0.03$

0.21

0.02

0.04

0.08

0.38

0.43

0.43

2.06

0.11

$0.42 *$

$0.51 *$

$0.37 *$

0.08

0.37

0.22

0.20

$-0.43$

$0.34 *$

$0.17 \quad 18$

100

$0.13 *$

$\begin{array}{rrrl}207 & 0 & 0.33 & * * * * \\ 158 & 236 & 0.17 & \\ 0 & 0 & 0.11 & \\ 217 & 435 & 0.23 & \\ 354 & 0 & 0.06 & \\ 26 & 1047 & 0.15 & \\ 19 & 729 & -0.02 & \\ 0 & 0 & -0.44 & * * * * \\ 47 & 0 & 0.34 & * * * \\ 245 & 257 & 0.46 & * * * * \\ 150 & 262 & 0.66 & * * * \\ 158 & 373 & -0.32 & \\ 0 & - & -0.67 & \\ 154 & 0 & 0.39 & \\ 0 & 0 & 0.09 & \\ - & - & - & \\ 38 & 191 & 0.25 & * * \\ - & - & - & \\ 49 & 174 & 0.27 & * * * *\end{array}$

$0.24 *$

$1.00 * * * *$

$0.37 *$

$0.32 *$

0.19

$-0.03$

$0.31 *$

$0.21 *$

$0.75 * *$

$0.80 * * * *$

$\begin{array}{lll}1.52 & 2 & 2\end{array}$

$0.85 \quad 0 \quad 0$

$\begin{array}{lll}0.50 & 0 & 0\end{array}$

$\begin{array}{lll}1.62 & 24 & 24\end{array}$

$\begin{array}{lll}0.23 & 2 & 11\end{array}$

$\begin{array}{lll}0.16 & 4 & 27\end{array}$

$\begin{array}{lll}0.30 & 8 & 29\end{array}$

$1.25 \quad 46 \quad 44$

$\begin{array}{lll}0.93 & 54 & 58\end{array}$

$\begin{array}{lll}1.70 & 53 \quad 42\end{array}$

$\begin{array}{lll}0.66 & 686 & 180\end{array}$

$\begin{array}{lll}0.62 & 12 & 23\end{array}$

$\begin{array}{lll}1.03 & 52 & 53\end{array}$

$0.63 \quad 67 \quad 86$

$0.16 \quad 45 \quad 212$

$\begin{array}{lll}0.78 & 8 & 14\end{array}$

$\begin{array}{lll}-0.06 & 45 & 0\end{array}$

$\begin{array}{lll}0.69 & 25 & 40\end{array}$

$\begin{array}{lll}0.58 & 22 & 41\end{array}$

$\begin{array}{lll}1.42 & 0 & 0\end{array}$

$0.77 \quad 40 \quad 54$

$0.36 * *$

0.17

$-$

0.35

$0.52 *$

$27 \quad 76$

172407

$\begin{array}{ll}45 & 287\end{array}$

$38 \quad 136$

$21 \quad 325$

$0 \quad 0$

$37 \quad 0$

230

$111 \quad 181$

$122 \quad 148$

$43 \quad 62$

180

$-\quad-$

$42 \quad 91$

$69 \quad 452$

- $\quad$

$0.23 * 26 \quad 93$

-

\begin{tabular}{lll}
$0.43 \quad * * * *$ & $53 \quad 147$ \\
\hline
\end{tabular} 
Table 7 continued

Experiment 3

\section{Phytoplankton}

Pseudonitzschia spp. (120 $\mu \mathrm{m})$

Pseudonitzschia spp. $(80 \mu \mathrm{m})$

Pseudonitzschia spp. $(60 \mu \mathrm{m})$

Navicula spp. $(40 \mu \mathrm{m})$

Navicula spp. $(20 \mu \mathrm{m})$

Navicula spp. $(10 \mu \mathrm{m})$

Chaetoceros danicus

Chaetoceros spp. $(40 \mu \mathrm{m})$

Chaetoceros spp. $(30 \mu \mathrm{m})$

Chaetoceros spp. $(20 \mu \mathrm{m})$

Chaetoceros spp. $(10 \mu \mathrm{m})$

Rhizosolenia styliformis/hebetata

Rhizosolenia pungens/setigera

Thalassiosira nordenskioeldii

Thalassiosira rotula

Flagellates $(25 \mu \mathrm{m})$

Flagellates $(20 \mu \mathrm{m})$

Flagellates $(15 \mu \mathrm{m})$

Flagellates $(10 \mu \mathrm{m})$

Flagellates $(5 \mu \mathrm{m})$

Total phytoplankton

\section{Microzooplankton}

Gyrodinium spp. (30-75 $\mu \mathrm{m})$

Gyrodinium spp. (75-120 $\mu \mathrm{m})$

Protoperidinium spp. $(20-40 \mu \mathrm{m})$

Protoperidinium spp. $(50-80 \mu \mathrm{m})$

Ceratium spp.

Torodinium spp.

Other athecate dinoflagellates

Other thecate dinoflagellates

Strombidium spp. $(25-40 \mu \mathrm{m})$

Strombidium spp. $(40-110 \mu \mathrm{m})$

Strobilidium spp.

Myrionecta rubra

Other Cyclotrichids

Haptorids

Tintinnids

Other Ciliates

Thecate amoeba sp.

Rotifers

Total microzooplankton

\begin{tabular}{|c|c|c|c|}
\hline-0.04 & & 0.34 & \\
\hline-0.05 & & 0.14 & \\
\hline-0.37 & & 0.09 & \\
\hline-0.28 & & 0.74 & $* * *$ \\
\hline-0.46 & & -0.01 & \\
\hline-0.39 & $*$ & 0.45 & $* *$ \\
\hline 0.02 & & 0.18 & \\
\hline-0.23 & & 0.35 & \\
\hline 0.19 & & -0.10 & \\
\hline-0.16 & & 0.56 & $* * *$ \\
\hline- & & - & \\
\hline-0.09 & & 0.02 & \\
\hline 0.06 & & 0.15 & \\
\hline-0.36 & $*$ & 0.04 & \\
\hline-0.34 & & -0.02 & \\
\hline-0.28 & & 1.40 & \\
\hline-0.51 & $*$ & 0.07 & \\
\hline-0.39 & $*$ & 0.42 & \\
\hline-0.15 & & -0.07 & \\
\hline-0.04 & & 0.27 & $* *$ \\
\hline-0.18 & & 0.10 & \\
\hline
\end{tabular}

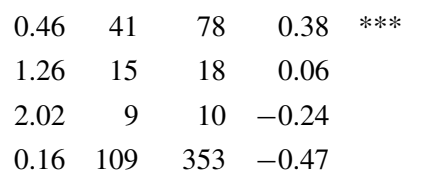

$0.45 \quad 0$

0.23

$\begin{array}{lll}0 & 0 & -0.36\end{array}$

$175-0.08$

$$
*
$$

$\begin{array}{rrrr}-0.02 & 19 & 0 & -0.19 \\ 1.72 & 42 & 36 & 0.23\end{array}$

$\begin{array}{lllll}0.50 & 0 & 0 & 0.32 & *\end{array}$

$\begin{array}{llll}0.08 & 75 & 555 & 0.38\end{array}$

$\begin{array}{llll}0 & - & - & -\end{array}$

$\begin{array}{llll}0.73 & 2 & 4 & 0.14\end{array}$

$\begin{array}{llll}0.74 & 16 & 27 & 0.03\end{array}$

$\begin{array}{llll}0.49 & 5 & 11 & 0.06\end{array}$

$\begin{array}{llll}1.14 & 0 & 0 & 0.21\end{array}$

$\begin{array}{llll}2.36 & 305 & 83 & 0.00\end{array}$

$\begin{array}{llll}0.76 & 7 & 12 & -0.21\end{array}$

$\begin{array}{llll}0.72 & 53 & 67 & -0.10\end{array}$

$0.95 \quad 0$

$\begin{array}{ll}0.76 & 32 \\ 0.65 & 11\end{array}$

$0 \quad-0.36$

$45-0.20$

0.65

\begin{tabular}{|c|c|c|c|}
\hline 0.02 & & 0.15 & $* *$ \\
\hline 0.20 & $*$ & 0.68 & $* * * *$ \\
\hline 0.03 & & 0.24 & \\
\hline 0.14 & & 0.91 & $* *$ \\
\hline 0.26 & & 0.50 & \\
\hline 0.27 & & 0.22 & \\
\hline
\end{tabular}

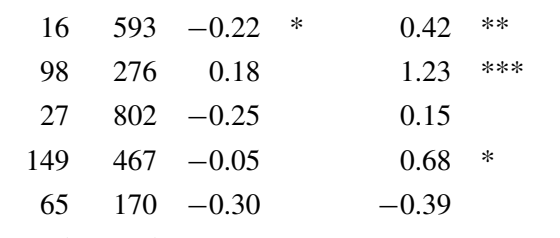

0.13
0.88 **
0.57
0.23
-0.11

$\begin{array}{rrr}0.75 & 14 & 23 \\ 1.82 & 140 & 70 \\ 1.46 & 78 & 57\end{array}$

$\begin{array}{lll}-0.20 \quad 26 & 0\end{array}$

$0.18 \quad 0 \quad 0$

$2.18 \quad 22 \quad 20$

$\begin{array}{lll}0.21 & 53 & 182\end{array}$

$\begin{array}{lll}1.67 & 61 & 47\end{array}$

$\begin{array}{lll}1.66 & 28 & 27\end{array}$

$0.68 \quad 0 \quad 0$

$\begin{array}{lll}0.68 & - & -\end{array}$

$\begin{array}{lll}0.65 & 4 & 7\end{array}$

$\begin{array}{rlrrr}0.42 & * * & 0.06 & 52 & 569 \\ 0.17 & * & 0.73 & 19 & 30\end{array}$

$\begin{array}{llll}0.24 & 1.20 & 27 & 30\end{array}$

$\begin{array}{llll}-1.05 & 3.04 & 0 & 0\end{array}$

$\begin{array}{llll}0.24 & 0.73 \quad 28 & 42\end{array}$

$\begin{array}{llll}-0.11 & 0.68 & 0 & 0\end{array}$

$\begin{array}{llll}-0.16 & -0.59 & 0 & 0\end{array}$

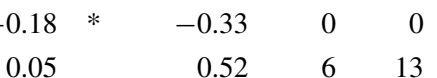

\begin{tabular}{rlrl}
- & & \multicolumn{1}{c}{-} & \\
-0.26 & & -0.02 & \\
-0.15 & & 1.34 & \\
-1.61 & $* * * *$ & 0.15 & \\
-0.61 & $* * * *$ & 0.22 & \\
-1.09 & $* * *$ & 0.34 & $*$ \\
0.96 & $*$ & 0.83 & \\
0.87 & $* *$ & 0.54 & $* *$ \\
0.26 & & 0.69 & \\
0.32 & & 0.27 & \\
-0.11 & & 0.13 & $*$ \\
0.02 & & 0.33 & \\
0.07 & & 0.38 & $* * *$
\end{tabular}

$25 \quad 85-$

$\begin{array}{rcccc}0 & - & - & & - \\ 0 & 0 & -0.47 & * & 0.06 \\ 284 & 0 & - & & -\end{array}$

$\begin{array}{llll}16 & 0 & 0.61 & 0.19\end{array}$

$\begin{array}{llllll}25 & 0 & -0.21 & 0.40 & 50 & 0\end{array}$

$\begin{array}{llllll}40 & 0 & -0.72 & 0.49 & 63 & 0\end{array}$

$\begin{array}{rrclrr}129 & 91 & - & - & - & - \\ 72 & 72 & -0.52 & 2.48 & 1097 & 0\end{array}$

$\begin{array}{lllllll}100 & 216 & - & & - & - & -\end{array}$

$\begin{array}{llllll}31 & 87 & -0.11 & 0.10 & 11 & 0\end{array}$

$\begin{array}{lllllll}13 & 0 & -0.05 & 0.25 & * * * & 29 & 0\end{array}$

$\begin{array}{lllllll}39 & 1183 & 0.03 & 1.84 & * * * & 529 & 3349\end{array}$

$\begin{array}{lllllll}46 & 478 & -0.04 & 0.78 & * * * * & 118 & 0\end{array}$

Negative $P_{i}$ and $P_{p}$ values resulting from negative $g\left(P_{i}\right)$ or $\mu_{o} / k\left(P_{p}\right)$ were set to zero, also positive $P_{p}$ values resulting from negative $g$ and $\mu_{o} / k$ $T C$ total T. longicornis carbon biomass

$P$ values derived from $t$ tests against zero, $* P<0.05$; ** $P<0.01$; *** $P<0.001$; **** $P<0.0001$ 
Table 8 Temora longicornis individual filtration $F_{i}\left(\mathrm{~mL} \mathrm{Ind.}^{-1}\right.$ day ${ }^{-1}$ ) and ingestion rates $I_{i}\left(\mathrm{ngC}\right.$ Ind. $^{-1}$ day $^{-1}$ ) as well as carbon-specific filtration rates $F_{c}\left(\mathrm{~mL} \mu \mathrm{gC}\right.$ predator ${ }^{-1}$ day $\left.^{-1}\right)$ and carbon-specific ingestion rates $I_{c}\left(\mu \mathrm{gC}\right.$ prey $\mu \mathrm{gC}$ predator $^{-1}$ day $^{-1}$ ), total ingestion rates $I_{\text {total }}$ $\left(\mu \mathrm{gC}\right.$ prey $\left.\mathrm{L}^{-1} \mathrm{day}^{-1}\right)$ and electivity $E^{*}(-)$ for each registered prey taxon

Temora longicornis $\left(\mathrm{TC} 108.70 \mu \mathrm{gC} \mathrm{L}^{-1}\right)$
\begin{tabular}{lllllllllllllllllll} 
Experiment 1 \\
\hline$F_{i}$ & $F_{c}$ & $P$ & $I_{i}$ & $I_{c}$ & $P$ & $E^{*}$ & $P$ & $F_{i}$ & $F_{c}$ & $P$ & $I_{i}$ & $I_{c}$ & $P$ & $E^{*}$ & $P$ \\
\hline
\end{tabular}

\begin{tabular}{|c|c|c|c|c|c|c|c|c|c|c|c|c|c|c|c|c|}
\hline \multicolumn{17}{|l|}{ Phytoplankton } \\
\hline $\begin{array}{l}\text { Pseudonitzschia spp. } \\
\quad(120 \mu \mathrm{m})\end{array}$ & -1.48 & -0.15 & & -1.65 & -0.0002 & & -0.80 & $* * *$ & 1.75 & 0.17 & & 1.16 & 0.0001 & & -0.71 & $* * *$ \\
\hline $\begin{array}{l}\text { Pseudonitzschia spp. } \\
\quad(80 \mu \mathrm{m})\end{array}$ & 6.80 & 0.68 & & -0.52 & -0.0001 & & -0.56 & $*$ & -3.24 & -0.32 & & -0.31 & 0.0000 & & -0.52 & $*$ \\
\hline $\begin{array}{l}\text { Pseudonitzschia spp. } \\
\quad(60 \mu \mathrm{m})\end{array}$ & 46.91 & 4.69 & & 0.44 & 0.0000 & & -0.52 & & -2.48 & -0.25 & & -0.33 & 0.0000 & & -0.48 & \\
\hline Navicula spp. $(40 \mu \mathrm{m})$ & 32.58 & 3.26 & & 1.12 & 0.0001 & & -0.22 & & 19.66 & 1.97 & & 0.98 & 0.0001 & & -0.49 & $*$ \\
\hline Navicula spp. $(20 \mu \mathrm{m})$ & 1.01 & 0.10 & & 0.90 & 0.0001 & & -0.58 & $* *$ & 2.10 & 0.21 & & 3.57 & 0.0004 & & -0.60 & $* * *$ \\
\hline Navicula spp. $(10 \mu \mathrm{m})$ & -5.31 & -0.53 & & -18.19 & -0.0018 & & -0.82 & $* * *$ & 3.86 & 0.39 & & 26.02 & 0.0026 & & -0.59 & $*$ \\
\hline Chaetoceros danicus & 92.80 & 9.28 & $* *$ & 122.09 & 0.0122 & $* *$ & 0.21 & & 7.30 & 0.73 & & 4.94 & 0.0005 & & -0.65 & $* *$ \\
\hline $\begin{array}{l}\text { Chaetoceros spp. } \\
(40 \mu \mathrm{m})\end{array}$ & 15.82 & 1.58 & & 4.50 & 0.0004 & & -0.40 & & 34.98 & 3.50 & & 17.82 & 0.0018 & & -0.18 & \\
\hline $\begin{array}{l}\text { Chaetoceros spp. } \\
\quad(30 \mu \mathrm{m})\end{array}$ & 26.53 & 2.65 & & 47.82 & 0.0048 & & -0.34 & & 40.01 & 4.00 & & 32.18 & 0.0032 & & -0.01 & \\
\hline $\begin{array}{l}\text { Chaetoceros spp. } \\
(20 \mu \mathrm{m})\end{array}$ & 37.28 & 3.73 & $* *$ & 219.95 & 0.0220 & $* *$ & -0.21 & & 39.20 & 3.92 & $*$ & 101.04 & 0.0101 & & -0.13 & \\
\hline $\begin{array}{l}\text { Chaetoceros spp. } \\
\quad(10 \mu \mathrm{m})\end{array}$ & -9.38 & -0.94 & & -24.59 & -0.0025 & & -0.86 & $* * * *$ & 189.72 & 18.97 & & 65.33 & 0.0065 & & 0.51 & \\
\hline $\begin{array}{l}\text { Rhizosolenia } \\
\quad \text { styliformis/hebetata }\end{array}$ & 11.88 & 1.19 & & 398.88 & 0.0399 & & -0.60 & $* *$ & 10.15 & 1.02 & & 316.50 & 0.0316 & & -0.46 & $*$ \\
\hline $\begin{array}{l}\text { Rhizosolenia pungens/ } \\
\text { setigera }\end{array}$ & 26.92 & 2.69 & & 70.84 & 0.0071 & & -0.01 & & 38.36 & 3.84 & $* *$ & 290.95 & 0.0291 & $* *$ & -0.16 & \\
\hline $\begin{array}{l}\text { Thalassiosira } \\
\text { nordenskioeldii }\end{array}$ & 15.47 & 1.55 & $*$ & 439.49 & 0.0439 & $*$ & -0.52 & $* * *$ & 47.27 & 4.73 & $*$ & 1311.92 & 0.1312 & $*$ & -0.07 & \\
\hline Thalassiosira rotula & 27.63 & 2.76 & $* *$ & 222.88 & 0.0223 & $* *$ & -0.32 & * & 33.88 & 3.39 & $*$ & 254.22 & 0.0254 & * & -0.15 & \\
\hline Flagellates $(25 \mu \mathrm{m})$ & 91.50 & 9.15 & & 28.93 & 0.0029 & & 0.12 & & 7.19 & 0.72 & & -14.08 & -0.0014 & & -0.42 & \\
\hline Flagellates $(20 \mu \mathrm{m})$ & 29.59 & 2.96 & & 11.03 & 0.0011 & & -0.12 & & 34.13 & 3.41 & & 36.32 & 0.0036 & & -0.31 & \\
\hline Flagellates $(15 \mu \mathrm{m})$ & 2.25 & 0.22 & & 8.44 & 0.0008 & & -0.44 & $* *$ & 20.61 & 2.06 & $* *$ & 38.86 & 0.0039 & $*$ & -0.34 & $*$ \\
\hline Flagellates $(10 \mu \mathrm{m})$ & 28.70 & 2.87 & & 82.87 & 0.0083 & & -0.29 & & 18.39 & 1.84 & & 45.95 & 0.0046 & & -0.43 & \\
\hline Flagellates $(5 \mu \mathrm{m})$ & -13.28 & -1.33 & & -134.07 & -0.0134 & & -0.82 & $* * * *$ & -39.27 & -3.93 & & -79.18 & -0.0079 & & -0.87 & $* * *$ \\
\hline Total phytoplankton & 15.51 & 1.55 & $* * *$ & 1643.64 & 0.1644 & $* * *$ & -0.28 & $* *$ & 31.21 & 3.12 & $*$ & 2973.72 & 0.2974 & $*$ & -0.28 & \\
\hline $\begin{array}{l}\text { Related to total } \\
\text { copepod C L }{ }^{-1}\end{array}$ & & & & $I_{\text {total }}$ & 17.87 & & & & & & & $I_{\text {total }}$ & 32.32 & & & \\
\hline \multicolumn{17}{|l|}{ Microzooplankton } \\
\hline $\begin{array}{l}\text { Gyrodinium spp. } \\
(30-75 \mu \mathrm{m})\end{array}$ & 103.21 & 10.32 & $* *$ & 236.46 & 0.0236 & $* *$ & 0.10 & & 22.17 & 2.22 & $* *$ & 281.39 & 0.028 & $* *$ & -0.27 & \\
\hline $\begin{array}{l}\text { Gyrodinium spp. } \\
\quad(75-120 \mu \mathrm{m})\end{array}$ & 87.07 & 8.71 & $* *$ & 27.29 & 0.0027 & $* *$ & 0.34 & & 91.98 & 9.20 & $* * * *$ & 52.12 & 0.0052 & $* * *$ & 0.41 & $* * *$ \\
\hline $\begin{array}{l}\text { Protoperidinium spp. } \\
\quad(20-40 \mu \mathrm{m})\end{array}$ & -13.29 & -1.33 & & -0.56 & -0.0001 & & -0.67 & $* *$ & 34.31 & 3.43 & $*$ & 9.84 & 0.0010 & & -0.23 & \\
\hline $\begin{array}{l}\text { Protoperidinium spp. } \\
\quad(50-80 \mu \mathrm{m})\end{array}$ & 106.09 & 10.61 & $* *$ & 81.36 & 0.0081 & $* *$ & 0.31 & & 29.62 & 2.96 & $*$ & 82.35 & 0.0082 & $*$ & -0.20 & \\
\hline Ceratium spp. & 139.27 & 13.93 & & 10.68 & 0.0011 & & 0.56 & & 17.68 & 1.77 & & 2.68 & 0.0003 & & -0.43 & \\
\hline Torodinium spp. & 21.37 & 2.14 & & 0.04 & 0.0000 & & -0.33 & & -2.91 & -0.29 & & -0.39 & 0.0000 & & -0.70 & $* *$ \\
\hline $\begin{array}{l}\text { Other athecate } \\
\text { dinoflagellates }\end{array}$ & 16.30 & 1.63 & & 19.47 & 0.0019 & & -0.50 & $* *$ & 28.82 & 2.88 & $*$ & 27.43 & 0.0027 & $*$ & -0.16 & \\
\hline $\begin{array}{l}\text { Other thecate } \\
\text { dinoflagellates }\end{array}$ & -2.59 & -0.26 & & -23.41 & -0.0023 & & -0.88 & $* * * *$ & 19.22 & 1.92 & $*$ & 139.51 & 0.0140 & $*$ & -0.40 & $*$ \\
\hline $\begin{array}{l}\text { Strombidium spp. } \\
\quad(25-40 \mu \mathrm{m})\end{array}$ & 35.67 & 3.57 & & 3.97 & 0.0004 & & -0.25 & & 68.57 & 6.86 & $* *$ & 9.15 & 0.0009 & $* *$ & 0.19 & \\
\hline $\begin{array}{l}\text { Strombidium spp. } \\
\quad(40-110 \mu \mathrm{m})\end{array}$ & 113.94 & 11.39 & $* * *$ & 242.60 & 0.0243 & $* * * *$ & 0.37 & $* *$ & 73.24 & 7.32 & $* * * *$ & 1091.25 & 0.1091 & $* * * *$ & 0.34 & $* * *$ \\
\hline
\end{tabular}


Table 8 continued

\begin{tabular}{|c|c|c|c|c|c|c|c|c|c|c|c|c|c|c|c|c|}
\hline & \multicolumn{16}{|c|}{ Temora longicornis (TC $108.70 \mu \mathrm{gC} \mathrm{L}^{-1}$ ) } \\
\hline & \multicolumn{8}{|c|}{ Experiment 1} & \multicolumn{8}{|c|}{ Experiment 2} \\
\hline & $F_{i}$ & $F_{c}$ & $P$ & $I_{i}$ & $I_{c}$ & $P$ & $E^{*}$ & $P$ & $F_{i}$ & $F_{c}$ & $P$ & $I_{i}$ & $I_{c}$ & $P$ & $E^{*}$ & $P$ \\
\hline Strobilidium spp. & 84.30 & 8.43 & $*$ & 42.07 & 0.0042 & $*$ & 0.04 & & 32.78 & 3.28 & $* *$ & 190.64 & 0.0191 & $* *$ & -0.15 & \\
\hline Myrionecta rubra & 87.06 & 8.71 & $* *$ & 179.10 & 0.0179 & $* * * *$ & 0.23 & $*$ & 15.47 & 1.55 & & 52.50 & 0.0052 & & -0.33 & \\
\hline Other Cyclotrichids & 0.00 & 0.00 & & - & - & & - & & - & - & & - & - & & - & \\
\hline Haptorids & 85.78 & 8.58 & $*$ & 7.02 & 0.0007 & $*$ & -0.08 & & 32.07 & 3.21 & & 2.06 & 0.0002 & & -0.16 & \\
\hline Tintinnids & -7.09 & -0.71 & & -0.47 & 0.0000 & & -0.40 & & 48.19 & 4.82 & $*$ & 11.43 & 0.0011 & $* *$ & 0.08 & \\
\hline Other Ciliates & - & - & & - & - & & - & & - & - & & - & - & & - & \\
\hline Thecate amoeba sp. & 29.76 & 2.98 & $* * *$ & 60.68 & 0.0061 & $* * *$ & -0.26 & $*$ & 21.60 & 2.16 & $*$ & 142.56 & 0.0143 & $*$ & -0.30 & \\
\hline Rotifers & - & - & & - & - & & - & & - & - & & - & - & & - & \\
\hline $\begin{array}{l}\text { Total } \\
\text { microzooplankton }\end{array}$ & 36.72 & 3.67 & $* * * *$ & 880.91 & 0.0881 & $* * * *$ & 0.15 & $* *$ & 39.33 & 3.93 & $* * * *$ & 2316.33 & 0.2316 & $* * * *$ & 0.04 & \\
\hline \multirow[t]{2}{*}{$\begin{array}{l}\text { Related to total } \\
\text { copepod } \mathrm{C} \mathrm{L}^{-1}\end{array}$} & & & & $I_{\text {total }}$ & 9.58 & & & & & & & $I_{\text {total }}$ & 25.18 & & & \\
\hline & \multicolumn{8}{|c|}{ Experiment 3} & \multicolumn{8}{|c|}{ Experiment 4} \\
\hline \multicolumn{17}{|l|}{ Phytoplankton } \\
\hline $\begin{array}{l}\text { Pseudonitzschia spp. } \\
\quad(120 \mu \mathrm{m})\end{array}$ & 31.39 & 3.14 & & 18.01 & 0.0018 & & -0.31 & & 11.74 & 1.17 & & 8.78 & 0.0009 & & -0.54 & $* *$ \\
\hline $\begin{array}{l}\text { Pseudonitzschia spp. } \\
\quad(80 \mu \mathrm{m})\end{array}$ & 12.71 & 1.27 & & 0.57 & 0.0001 & & -0.49 & $*$ & 80.56 & 8.06 & $* *$ & 3.13 & 0.0003 & $* *$ & 0.16 & \\
\hline $\begin{array}{l}\text { Pseudonitzschia spp. } \\
\quad(60 \mu \mathrm{m})\end{array}$ & 8.16 & 0.82 & & 0.07 & 0.0000 & & -0.60 & & 52.85 & 5.28 & & 0.23 & 0.0000 & & 0.00 & \\
\hline Navicula spp. $(40 \mu \mathrm{m})$ & 68.03 & 6.80 & $* * *$ & 2.20 & 0.0002 & $* *$ & 0.22 & & 21.06 & 2.11 & & 0.61 & 0.0001 & & -0.37 & \\
\hline Navicula spp. $(20 \mu \mathrm{m})$ & -0.48 & -0.05 & & -2.95 & -0.0003 & & -0.65 & $* *$ & -10.07 & -1.01 & & -2.23 & -0.0002 & & -0.68 & $* *$ \\
\hline Navicula spp. $(10 \mu \mathrm{m})$ & 41.17 & 4.12 & $* *$ & 13.65 & 0.0014 & $*$ & -0.10 & & 18.42 & 1.84 & & -0.36 & 0.0000 & & -0.54 & \\
\hline Chaetoceros danicus & 16.32 & 1.63 & & 23.65 & 0.0024 & & -0.42 & & 39.14 & 3.91 & & 35.57 & 0.0036 & & -0.16 & \\
\hline $\begin{array}{l}\text { Chaetoceros spp. } \\
\qquad(40 \mu \mathrm{m})\end{array}$ & 32.00 & 3.20 & & 14.46 & 0.0014 & & -0.35 & & 43.83 & 4.38 & & 10.20 & 0.0010 & & -0.27 & \\
\hline $\begin{array}{l}\text { Chaetoceros spp. } \\
\qquad(30 \mu \mathrm{m})\end{array}$ & -9.08 & -0.91 & & -93.07 & -0.0093 & & -0.76 & $* * * *$ & 22.52 & 2.25 & & 26.28 & 0.0026 & & -0.41 & \\
\hline $\begin{array}{l}\text { Chaetoceros spp. } \\
\qquad(20 \mu \mathrm{m})\end{array}$ & 51.58 & 5.16 & $* * *$ & 259.00 & 0.0259 & $* *$ & 0.09 & & -8.80 & -0.88 & & -91.85 & -0.0092 & & -0.72 & $* * *$ \\
\hline $\begin{array}{l}\text { Chaetoceros spp. } \\
\qquad(10 \mu \mathrm{m})\end{array}$ & - & - & & - & - & & - & & - & - & & - & - & & - & \\
\hline $\begin{array}{l}\text { Rhizosolenia } \\
\quad \text { styliformis/hebetata }\end{array}$ & 1.92 & 0.19 & & 48.73 & 0.0049 & & -0.73 & $* * *$ & 3.27 & 0.33 & & 21.17 & 0.0021 & & -0.72 & $* *$ \\
\hline $\begin{array}{l}\text { Rhizosolenia pungens/ } \\
\text { setigera }\end{array}$ & 13.87 & 1.39 & & 40.93 & 0.0041 & & -0.45 & $*$ & 38.32 & 3.83 & $* *$ & 54.55 & 0.0055 & $*$ & -0.14 & \\
\hline $\begin{array}{l}\text { Thalassiosira } \\
\text { nordenskioeldii }\end{array}$ & 4.11 & 0.41 & & 10.62 & 0.0011 & & -0.66 & $* *$ & 15.73 & 1.57 & $*$ & 303.59 & 0.0304 & $*$ & -0.42 & $*$ \\
\hline Thalassiosira rotula & -1.52 & -0.15 & & -24.78 & -0.0025 & & -0.60 & $*$ & 21.93 & 2.19 & & 54.55 & 0.0055 & & -0.48 & $*$ \\
\hline Flagellates $(25 \mu \mathrm{m})$ & 128.74 & 12.87 & & 18.61 & 0.0019 & & 0.56 & & -97.03 & -9.70 & & -12.83 & -0.0013 & & -1.00 & \\
\hline Flagellates $(20 \mu \mathrm{m})$ & 6.06 & 0.61 & & 1.18 & 0.0001 & & -0.41 & & 22.41 & 2.24 & & 4.36 & 0.0004 & & -0.19 & \\
\hline Flagellates $(15 \mu \mathrm{m})$ & 39.00 & 3.90 & & 5.40 & 0.0005 & & -0.15 & & -10.54 & -1.05 & & -4.98 & -0.0005 & & -0.70 & $* *$ \\
\hline Flagellates $(10 \mu \mathrm{m})$ & -6.63 & -0.66 & & -5.42 & -0.0005 & & -0.82 & $* * *$ & -14.91 & -1.49 & & -113.03 & -0.0113 & & -0.86 & $* * * *$ \\
\hline Flagellates $(5 \mu \mathrm{m})$ & 25.29 & 2.53 & $* *$ & 310.09 & 0.0310 & $* *$ & -0.28 & & -16.17 & -1.62 & $*$ & -223.63 & -0.0224 & $*$ & -0.95 & $* * * *$ \\
\hline Total phytoplankton & 9.34 & 0.93 & & 866.25 & 0.0866 & & -0.45 & $*$ & 4.97 & 0.50 & & 370.16 & 0.0370 & & -0.74 & $* * *$ \\
\hline $\begin{array}{l}\text { Related to total } \\
\text { copepod } \mathrm{C} \mathrm{L}^{-1}\end{array}$ & & & & $I_{\text {total }}$ & 9.42 & & & & & & & $I_{\text {total }}$ & 4.02 & & & \\
\hline \multicolumn{17}{|l|}{ Microzooplankton } \\
\hline $\begin{array}{l}\text { Gyrodinium spp. } \\
\qquad(30-75 \mu \mathrm{m})\end{array}$ & 13.89 & 1.39 & $* *$ & 206.51 & 0.0207 & $* *$ & -0.47 & $* * *$ & 38.68 & 3.87 & $* *$ & 229.15 & 0.0229 & $* * *$ & -0.15 & \\
\hline $\begin{array}{l}\text { Gyrodinium spp. } \\
\qquad(75-120 \mu \mathrm{m})\end{array}$ & 62.84 & 6.28 & $* * * *$ & 106.13 & 0.0106 & $* * * *$ & 0.24 & $* *$ & 113.01 & 11.30 & $* * *$ & 175.73 & 0.0176 & $* * * *$ & 0.44 & $* * *$ \\
\hline
\end{tabular}


Table 8 continued

\begin{tabular}{|c|c|c|c|c|c|c|c|c|c|c|c|c|c|c|c|c|}
\hline & \multicolumn{16}{|c|}{ Temora longicornis $\left(\mathrm{TC} 108.70 \mu \mathrm{gC} \mathrm{L}^{-1}\right.$ ) } \\
\hline & \multicolumn{8}{|c|}{ Experiment 1} & \multicolumn{8}{|c|}{ Experiment 2} \\
\hline & $F_{i}$ & $F_{c}$ & $P$ & $I_{i}$ & $I_{c}$ & $P$ & $E^{*}$ & $P$ & $F_{i}$ & $F_{c}$ & $P$ & $I_{i}$ & $I_{c}$ & $P$ & $E^{*}$ & $P$ \\
\hline $\begin{array}{l}\text { Protoperidinium spp. } \\
\quad(20-40 \mu \mathrm{m})\end{array}$ & 22.11 & 2.21 & & 2.04 & 0.0002 & & -0.46 & & 13.45 & 1.34 & & 0.65 & 0.0001 & & -0.52 & $*$ \\
\hline $\begin{array}{l}\text { Protoperidinium spp. } \\
\quad(50-80 \mu \mathrm{m})\end{array}$ & 83.79 & 8.38 & $* *$ & 99.18 & 0.0099 & $* *$ & 0.25 & & 62.94 & 6.29 & $*$ & 61.92 & 0.0062 & $*$ & 0.02 & \\
\hline Ceratium spp. & 46.21 & 4.62 & & 4.47 & 0.0004 & & -0.06 & & -36.28 & -3.63 & & -12.94 & -0.0013 & & -1.00 & \\
\hline Torodinium spp. & 20.41 & 2.04 & & 1.43 & 0.0001 & & -0.54 & & - & - & & - & - & & - & \\
\hline $\begin{array}{l}\text { Other athecate } \\
\text { dinoflagellates }\end{array}$ & - & - & & - & - & & - & & - & - & & - & - & & - & \\
\hline $\begin{array}{l}\text { Other thecate } \\
\text { dinoflagellates }\end{array}$ & -1.89 & -0.19 & & -7.54 & -0.0008 & & -0.34 & & 5.39 & 0.54 & & 0.14 & 0.0000 & & -0.63 & $*$ \\
\hline $\begin{array}{l}\text { Strombidium spp. } \\
\qquad(25-40 \mu \mathrm{m})\end{array}$ & 123.67 & 12.37 & & 5.06 & 0.0005 & & 0.42 & & - & - & & - & - & & - & \\
\hline $\begin{array}{l}\text { Strombidium spp. } \\
\qquad(40-110 \mu \mathrm{m})\end{array}$ & 13.41 & 1.34 & & -173.09 & -0.0173 & & -0.53 & & 17.03 & 1.70 & & - & - & & - & \\
\hline Strobilidium spp. & 20.27 & 2.03 & & 85.21 & 0.0085 & & -0.38 & & 37.08 & 3.71 & & 1.23 & 0.0001 & & -0.18 & \\
\hline Myrionecta rubra & 30.94 & 3.09 & $*$ & 15.96 & 0.0016 & & -0.20 & & 45.00 & 4.50 & & -7.01 & -0.0007 & & -0.14 & \\
\hline Other Cyclotrichids & 76.40 & 7.64 & & 18.11 & 0.0018 & & 0.00 & & - & - & & - & - & & - & \\
\hline Haptorids & 49.72 & 4.97 & $* *$ & 1116.13 & 0.1116 & & -0.10 & & 228.35 & 22.83 & & 198.93 & 0.0199 & & 0.19 & \\
\hline Tintinnids & 63.73 & 6.37 & & 5.65 & 0.0006 & & 0.24 & & - & - & & - & - & & - & \\
\hline Other Ciliates & 24.86 & 2.49 & & -2.36 & -0.0002 & & -0.05 & & 9.3870 & 0.94 & & 29.6777 & 0.0030 & & -0.58 & $* *$ \\
\hline Thecate amoeba sp. & 11.55 & 1.16 & $*$ & 46.54 & 0.0047 & $*$ & -0.52 & $* *$ & 23.21 & 2.32 & $* * *$ & 39.28 & 0.0039 & $* * *$ & -0.33 & $*$ \\
\hline Rotifers & 30.55 & 3.06 & & 47.86 & 0.0048 & & -0.22 & & 169.13 & 16.91 & $* * *$ & 706.47 & 0.0706 & $* * *$ & 0.53 & $* * *$ \\
\hline $\begin{array}{l}\text { Total } \\
\text { microzooplankton }\end{array}$ & 35.02 & 3.50 & $* * *$ & 2649.93 & 0.2650 & $* *$ & 0.18 & $*$ & 71.61 & 7.16 & $* * * *$ & 1635.56 & 0.1636 & $* * * *$ & 0.28 & $* * * *$ \\
\hline $\begin{array}{l}\text { Related to total } \\
\text { copepod } \mathrm{C} \mathrm{L}^{-1}\end{array}$ & & & & $I_{\text {total }}$ & 28.80 & & & & & & & $I_{\text {total }}$ & 17.78 & & & \\
\hline
\end{tabular}

Positive selection given in italic values

$T C$ total T. longicornis carbon biomass

$P$ values derived from $t$ tests against zero, ${ }^{*} P<0.05$, ** $P<0.01$, *** $P<0.001$, **** $P<0.0001$

\section{References}

Aberle N, Lengfellner K, Sommer U (2007) Spring bloom succession, grazing impact and herbivore selectivity of ciliate communities in response to winter warming. Oecologia 150:668-681

Arndt H (1993) Rotifers as predators on components of the microbial web (bacteria, heterotrophic flagellates, ciliates) - a review. Hydrobiologia 255:231-246

Azam F, Fenchel T, Field JG, Gray JS, Meyer-Reil LA, Thingstad F (1983) The ecological role of water-column microbes in the sea. Mar Ecol Prog Ser 10:257-263

Båmstedt U, Gifford DJ, Irigoien X, Atkinson A, Roman M (2000) Feeding. In: Harris R, Wiebe P, Lenz J, Skjoldal HR, Huntley M (eds) ICES zooplankton methodology manual. Academic Press, London, pp 297-399

Bec A, Martin-Creuzburg D, von Elert E (2006) Trophic upgrading of autotrophic picoplankton by the heterotrophic nanoflagellate Paraphysomonas sp. Limnol Oceanogr 51:1699-1707

Brock TD (1981) Calculating solar radiation for ecological studies. Ecolog Model 14:1-19

Broglio E, Jónasdóttir SH, Calbet A, Jakobsen HH, Saiz E (2003) Effect of heterotrophic versus autotrophic food on feeding and reproduction of the calanoid copepod Acartia tonsa: relationship with prey fatty acid composition. Aquat Microb Ecol 31:267-278
Brussaard CPD, Riegman R, Noordeloos AAM, Cadee GC, Witte H, Kop AJ, Nieuwland G, Vanduyl FC, Bak RPM (1995) Effects of grazing, sedimentation and phytoplankton cell lysis on the structure of a coastal pelagic food web. Mar Ecol Prog Ser 123:259-271

Calbet A (2001) Mesozooplankton grazing effect on primary production: a global comparative analysis in marine ecosystems. Limnol Oceanogr 46:1824-1830

Calbet A, Landry MR (2004) Phytoplankton growth, microzooplankton grazing, and carbon cycling in marine systems. Limnol Oceanogr 49:51-57

Calbet A, Saiz E (2005) The ciliate-copepod link in marine ecosystems. Aquat Microb Ecol 38:157-167

Carey PG (1992) Marine interstitial ciliates: an illustrated key. Chapman \& Hall, London

Caron DA (2000) Protistan herbivory and bacterivory. In: Paul J (ed) Methods in microbiology, vol 30. Academic Press, San Diego, pp 289-315

Chesson J (1978) Measuring preference in selective predation. Ecol 59:211-215

Chesson J (1983) The estimation and analysis of preference and its relationship to foraging models. Ecol 64:1297-1304

Christaki U, Dolan JR, Pelegri S, Rassoulzadegan F (1998) Consumption of picoplankton-size particles by marine ciliates: 
effects of physiological state of the ciliate and particle quality. Limnol Oceanogr 43:458-464

Cowles TJ, Olson RJ, Chisholm SW (1988) Food selection by copepods-discrimination on the basis of food quality. Mar Biol 100:41-49

Crawford DW, Stoecker DK (1996) Carbon content, dark respiration and mortality of the mixotrophic planktonic ciliate Strombidium capitatum. Mar Biol 126:415-422

Dagg MJ, Vidal J, Whitledge TE, Iverson RL, Goering JJ (1982) The feeding, respiration, and excretion of zooplankton in the Bering Sea during a spring bloom. Deep-Sea Res Part I: Oceanogr Res Pap 29:45-63

Dam HG (1986) Short-term feeding of Temora longicornis Müller in the laboratory and the field. J Exp Mar Biol Ecol 99:149-161

Dam HG, Peterson WT (1993) Seasonal contrasts in the diel vertical distribution, feeding-behavior, and grazing impact of the copepod Temora longicornis in Long-Island Sound. J Mar Res 51:561-594

Dam HG, Miller CA, Jonasdottir SH (1993) The trophic role of mesozooplankton at $47^{\circ} \mathrm{N}, 20^{\circ} \mathrm{W}$ during the North Atlantic Bloom Experiment. Deep-Sea Res Part II: Top Stud Oceanogr 40:197-212

Daro MH (1985) Field-study of selectivity, efficiency and daily variation in the feeding of the marine copepod Temora longicornis, in the Southern Bight of the North Sea. Bull Mar Sci 37:764-764

Dodge JD (1982) Marine dinoflagellates of the British Isles. Her Majesty's Stationery Office, London

Dolan JR, McKeon K (2005) The reliability of grazing rate estimates from dilution experiments: have we over-estimated rates of organic carbon consumption by microzooplankton? Ocean Sci $1: 1-7$

Dolan JR, Gallegos CL, Moigis A (2000) Dilution effects on microzooplankton in dilution grazing experiments. Mar Ecol Prog Ser 200:127-139

Du Yoo Y, Jeong HJ, Kim MS, Kang NS, Song JY, Shin W, Kim KY, Lee K (2009) Feeding by phototrophic red-tide dinoflagellates on the ubiquitous marine diatom Skeletonema costatum. J Eukaryot Microbiol 56:413-420

Fenchel T (1980) Relation between particle size selection and clearance in suspension-feeding ciliates. Limnol Oceanogr 25:733-738

Fenchel T, Finlay BJ (1983) Respiration rates in heterotrophic, freeliving protozoa. Microb Ecol 9:99-122

Fileman E, Smith T, Harris R (2007) Grazing by Calanus helgolandicus and Para-Pseudocalanus spp. on phytoplankton and protozooplankton during the spring bloom in the Celtic Sea. J Exp Mar Biol Ecol 348:70-84

First MR, Miller HL, Lavrentyev PJ, Pinckney JL, Burd AB (2009) Effects of microzooplankton growth and trophic interactions on herbivory in coastal and offshore environments. Aquat Microb Ecol 54:255-267

Flynn KJ (2008) Attack is not the best form of defense: Lessons from harmful algal bloom dynamics. Harmful Algae 8:129-139

Fonda Umani S, Tirelli V, Beran A, Guardiani B (2005) Relations between microzooplankton and mesozooplankton: Competition versus predation on natural assemblages of the Gulf of Trieste (Northern Adriatic Sea). J Plankton Res 27:973-986

Frost BW (1972) Effects of size and concentration of food particles on the feeding behaviour of the marine planktonic copepod Calanus pacificus. Limnol Oceanogr 17:805-815

Gaines G, Elbrächter M (1987) Heterotrophic nutrition. In: Taylor FJR (ed) The biology of dinoflagellates. Blackwell, Oxford, pp 224-268
Gallegos CL (1989) Microzooplankton grazing on phytoplankton in the Rhode River, Maryland: Nonlinear feeding kinetics. Mar Ecol Prog Ser 57:23-33

Gentsch E, Kreibich T, Hagen W, Niehoff B (2009) Dietary shifts in the copepod Temora longicornis during spring: evidence from stable isotope signatures, fatty acid biomarkers and feeding experiments. J Plankton Res 31:45-60

Gifford DJ (1985) Laboratory culture of marine planktonic oligotrichs (Ciliophora, Oligotrichida). Mar Ecol Prog Ser 23:257-267

Gifford DJ, Dagg MJ (1988) Feeding of the estuarine copepod Acartia tonsa dana: Carnivory vs. herbivory in natural microplankton assemblages. Bull Mar Sci 43:458-468

Grasshoff K, Kremling K, Ehrhardt M (eds) (1999) Methods of seawater analysis, 3rd edn. Wiley-VCH Verlag, Weinheim

Greve W, Reiners F, Nast J, Hoffmann S (2004) Helgoland Roads meso- and macrozooplankton time-series 1974 to 2004: lessons from 30 years of single spot, high frequency sampling at the only off-shore island of the North Sea. Helgol Mar Res 58:274-288

Guillard RRL, Ryther JH (1962) Studies of marine planktonic diatoms. I. Cyclotella nana Hustedt and Detonula confervacea (Cleve) Gran. Can J Microbiol 8:229-239

Hamels I, Mussche H, Sabbe K, Muylaert K, Vyverman W (2004) Evidence for constant and highly specific active food selection by benthic ciliates in mixed diatoms assemblages. Limnol Oceanogr 49:58-68

Hansen PJ (1992) Prey size selection, feeding rates and growth dynamics of heterotrophic dinoflagellates with special emphasis on Gyrodinium spirale. Mar Biol 114:327-334

Hansen FC, Reckermann M, Klein Breteler WCM, Riegman R (1993) Phaeocystis blooming enhanced by copepod predation on protozoa-evidence from incubation experiments. Mar Ecol Prog Ser 102:51-57

Heinbokel JF (1978) Studies on the functional role of tintinnids in the southern California Bight. I. Grazing and growth rates in laboratory cultures. Mar Biol 47:177-189

Hickel W, Mangelsdorf P, Berg J (1993) The human impact in the German Bight: eutrophication during three decades (1962-1991). Helgol Meeresunters 47:243-263

Hillebrand H, Dürselen C-D, Kirschtel D, Pollingher U, Zohary T (1999) Biovolume calculation for pelagic and benthic microalgae. J Phycol 35:403-424

Hoppenrath M, Elbrächter M, Drebes G (2009) Marine phytoplankton: Selected microphytoplankton species from the North Sea around Helgoland and Sylt. Kleine Senckenberg-Reihe 49. E. Schweizerbart'sche Verlagsbuchhandlung (Nägele u. Obermiller), Stuttgart

Irigoien X, Flynn KJ, Harris RP (2005) Phytoplankton blooms: a 'loophole' in microzooplankton grazing impact? J Plankton Res 27:313-321

Jackson KM, Berger J (1985) Survivorship curves of ciliate protozoa under starvation conditions and at low bacterial levels. Protistologica 21:17-24

Jacobson DM, Anderson DM (1986) Thecate heterotrophic dinoflagellates: feeding behavior and mechanisms. J Phycol 22:249258

Jakobsen HH, Halvorsen E, Hansen BW, Visser AW (2005) Effects of prey motility and concentration on feeding in Acartia tonsa and Temora longicornis: the importance of feeding modes. J Plankton Res 27(8):775-785

Jensen TC, Hessen DO (2007) Does excess dietary carbon affect respiration of Daphnia? Oecologia 152:191-200

Jeong HJ (1999) The ecological role of heterotrophic dinoflagellates in marine plankton community. $\mathrm{J}$ Eukaryot Microbiol 46:390-396 
Johansson M, Gorokhova E, Larsson U (2004) Annual variability in ciliate community structure, potential prey and predators in the open northern Baltic Sea proper. J Plankton Res 26:67-80

Jonsson PR (1986) Particle size selection, feeding rates and growth dynamics of marine planktonic oligotrichous ciliates (Ciliophora: Oligotrichina). Mar Ecol Prog Ser 33:265-277

Kahl A (1932) Urtiere oder Protozoa. I Wimpertiere. 3 Spirotricha. In: Dahl F (ed) Die Tierwelt Deutschlands und der angrenzenden Meeresteile, vol 25. Gustav Fischer, Jena

Kim YO, Ha S, Taniguchi A (2008) Morphology and in situ sedimentation of the cysts of a planktonic oligotrich ciliate, Strombidium capitatum. Aquat Microb Ecol 53:173-179

Klein Breteler WCM, Schogt N, Baas M, Schouten S, Kraay GW (1999) Trophic upgrading of food quality by protozoans enhancing copepod growth: role of essential lipids. Mar Biol 135:191-198

Kleppel GS (1993) On the diets of calanoid copepods. Mar Ecol Prog Ser 99:183-195

Kleppel GS, Holliday DV, Pieper RE (1991) Trophic interactions between copepods and microplankton: a question about the role of diatoms. Limnol Oceanogr 36:172-178

Köhler W, Schachtel G, Voleske P (1995) Biostatistik: Einführung in die Biometrie für Biologen und Agrarwissenschaftler, 2 edn edn. Springer, Berlin

Koski M, Dutz J, Klein Breteler WCM (2005) Selective grazing of temora longicornis in different stages of a Phaeocystis globosa bloom - a mesocosm study. Harmful Algae 4(5):915-927

Landry MR (1993) Estimating rates of growth and grazing mortality of phytoplankton by the dilution method. In: Kemp PF, Sherr BF, Sherr EB, Cole JJ (eds) Handbook of methods in aquatic microbial ecology. Lewis Publishers, Boca Raton, pp 715-722

Landry MR, Calbet A (2004) Microzooplankton production in the oceans. ICES J Mar Sci 61:501-507

Landry MR, Calbet A (2005) Reality checks on microbial food web interactions in dilution experiments: responses to the comments of Dolan and McKeon. Ocean Science 1:39-44

Landry MR, Hassett RP (1982) Estimating the grazing impact of marine micro-zooplankton. Mar Biol 67:283-288

Landry MR, Kirshtein J, Constantinou J (1995) A refined dilution technique for measuring the community grazing impact of microzooplankton, with experimental tests in the central equatorial Pacific. Mar Ecol Prog Ser 120:53-63

Löder MGJ (2010) The role of heterotrophic dinoflagellate and ciliate grazers in the food web at Helgoland Roads, North Sea. Dissertation, Jacobs University Bremen

Löder MGJ, Aberle N, Klaas C, Kraberg AC, Wiltshire KH (2010) Conserving original in situ diversity in microzooplankton grazing set-ups. Mar Biodivers Rec 3:e28

Löder MGJ, Kraberg AC, Aberle N, Peters S, Wiltshire KH (2011) Dinoflagellates and ciliates at Helgoland Roads, North Sea. Helgol Mar Res (in Press)

Maar M, Nielsen TG, Gooding S, Tonnesson K, Tiselius P, Zervoudaki S, Christou E, Sell A, Richardson K (2004) Trophodynamic function of copepods, appendicularians and protozooplankton in the late summer zooplankton community in the Skagerrak. Mar Biol 144:917-933

Malzahn AM, Hantzsche F, Schoo KL, Boersma M, Aberle N (2010) Differential effects of nutrient-limited primary production on primary, secondary or tertiary consumers. Oecologia 162:3548

Martin-Creuzburg D, Bec A, von Elert E (2005) Trophic upgrading of picocyanobacterial carbon by ciliates for nutrition of Daphnia magna. Aquat Microb Ecol 41:271-280

Mauchline J (1998) The biology of calanoid copepods. In: Advances in marine biology, vol 33. Academic Press, London
McCauley E (1984) The estimation of the abundance and biomass of zooplankton in samples. In: Downing JA, Rigler FH (eds) A manual on methods for the assessment of secondary productivity in freshwater, IBP Handbook no. 17, 2nd edn. Blackwell Scientific Publications, Oxford, pp 228-265

Menden-Deuer S, Lessard EJ (2000) Carbon to volume relationships for dinoflagellates, diatoms, and other protist plankton. Limnol Oceanogr 45:569-579

Menden-Deuer S, Lessard EJ, Satterberg J, Grünbaum D (2005) Growth rates and starvation survival of three species of the pallium-feeding, thecate dinoflagellate genus Protoperidinium. Aquat Microb Ecol 41:145-152

Moigis AG (2006) The clearance rate of microzooplankton as the key element for describing estimated non-linear dilution plots demonstrated by a model. Mar Biol 149:743-762

Montagnes DJS (2003) Planctonic ciliate project internet homepage. http://www.liv.ac.uk/ciliate/intro.htm. Accessed 19 December 2007

Montagnes DJS, Lessard EJ (1999) Population dynamics of the marine planktonic ciliate Strombidinopsis multiauris: Its potential to control phytoplankton blooms. Aquat Microb Ecol 20:167-181

Müller H, Geller W (1993) Maximum growth rates of aquatic ciliated protozoa-the dependence on body size and temperature reconsidered. Arch Hydrobiol 126:315-327

Müller H, Weisse T (1994) Laboratory and field observations on the scuticociliate Histiobalantium from the pelagic zone of Lake Constance, FRG. J Plankton Res 16:391-401

Naustvoll LJ (2000a) Prey size spectra and food preferences in thecate heterotrophic dinoflagellates. Phycologia 39:187-198

Naustvoll LJ (2000b) Prey size spectra in naked heterotrophic dinoflagellates. Phycologia 39:448-455

Nejstgaard JC, Gismervik I, Solberg PT (1997) Feeding and reproduction by Calanus finmarchicus, and microzooplankton grazing during mesocosm blooms of diatoms and the coccolithophore Emiliania huxleyi. Mar Ecol Prog Ser 147:197-217

Nejstgaard JC, Naustvoll L-J, Sazhin A (2001) Correcting for underestimation of microzooplankton grazing in bottle incubation experiments with mesozooplankton. Mar Ecol Prog Ser 221:59-75

O'Connors HB, Biggs DC, Ninivaggi DV (1980) Particle-sizedependent maximum grazing rates for Temora longicornis fed natural particle assemblages. Mar Biol 56(1):65-70

Paffenhöfer G-A (1988) Feeding rates and behavior of zooplankton. Bull Mar Sci 43:430-445

Park GS, Marshall HG (2000) The trophic contributions of rotifers in tidal freshwater and estuarine habitats. Estuar Coast Shelf Sci 51:729-742

Paterson HL, Knott B, Koslow AJ, Waite AM (2008) The grazing impact of microzooplankton off south west Western Australia: as measured by the dilution technique. J Plankton Res 30:379-392

Poole HH, Atkins WRG (1929) Photo-electric measurements of submarine illumination throughout the year. J Mar Biol Assoc UK 16:297-324

Putt M, Stoecker DK (1989) An experimentally determined carbon:volume ratio for marine "oligotrichous" ciliates from estuarine and coastal waters. Limnol Oceanogr 34:1097-1103

Quinlan EL, Jett CH, Phlips EJ (2009) Microzooplankton grazing and the control of phytoplankton biomass in the Suwannee River estuary, USA. Hydrobiologia 632:127-137

Riegman R, Kuipers BR, Noordeloos AAM, Witte HJ (1993) Sizedifferential control of phytoplankton and the structure of plankton communities. Neth J Sea Res 31:255-265

Schoo KL (2010) Stoichiometric constraints in primary producers affect secondary consumers. Dissertation, Christian-AlbrechtsUniversität, Kiel 
Sherr EB, Sherr BF (2002) Significance of predation by protists in aquatic microbial food webs. Antonie Van Leeuwenhoek Int J Gen Mol Microb 81:293-308

Sherr EB, Sherr BF (2007) Heterotrophic dinoflagellates: a significant component of microzooplankton biomass and major grazers of diatoms in the sea. Mar Ecol Prog Ser 352:187-197

Sherr EB, Sherr BF (2009) Capacity of herbivorous protists to control initiation and development of mass phytoplankton blooms. Aquat Microb Ecol 57:253-262

Sherr EB, Sherr BF, Paffenhöfer G-A (1986) Phagotrophic protozoa as food for metazoans: a "missing" trophic link in marine pelagic food webs? Mar Microb Food Webs 1:61-80

Sime-Ngando T, Demers S, Juniper SK (1999) Protozoan bacterivory in the ice and the water column of a cold temperate lagoon. Microb Ecol 37:95-106

Smetacek V (1981) The annual cycle of proto-zooplankton in the Kiel Bight. Mar Biol 63:1-11

Sommer U, Sommer F (2006) Cladocerans versus copepods: The cause of contrasting top-down controls on freshwater and marine phytoplankton. Oecologia 147:183-194

Sommer U, Sommer F, Santer B, Zöllner E, Jürgens K, Jamieson C, Boersma M, Gocke K (2003) Daphnia versus copepod impact on summer phytoplankton: Functional compensation at both trophic levels. Oecologia 135:639-647

Sommer F, Saage A, Santer B, Hansen T, Sommer U (2005a) Linking foraging strategies of marine calanoid copepods to patterns of nitrogen stable isotope signatures in a mesocosm study. Mar Ecol Prog Ser 286:99-106

Sommer U, Hansen T, Blum O, Holzner N, Vadstein O, Stibor H (2005b) Copepod and microzooplankton grazing in mesocosms fertilised with different $\mathrm{Si}: \mathrm{N}$ ratios: No overlap between food spectra and $\mathrm{Si}: \mathrm{N}$ influence on zooplankton trophic level. Oecologia 142:274-283

Sommer U, Aberle N, Engel A, Hansen T, Lengfellner K, Sandow M, Wohlers J, Zöllner E, Riebesell U (2007) An indoor mesocosm system to study the effect of climate change on the late winter and spring succession of Baltic Sea phyto- and zooplankton. Oecologia 150:655-667

Spittler P (1973) Feeding experiments with tintinnids. Oikos (Suppl.) 15:128-132

Stoecker DK, Capuzzo JM (1990) Predation on protozoa: its importance to zooplankton. J Plankton Res 12:891-908

Stoecker DK, Silver MW (1990) Replacement and aging of chloroplasts in Strombidium capitatum (Ciliophora, Oligotrichida). Mar Biol 107:491-502

Stoecker D, Guillard RRL, Kavee RM (1981) Selective predation by Favella ehrenbergii (Tintinnia) on and among dinoflagellates. Biolog Bull 160:136-145

Strom SL, Morello TA (1998) Comparative growth rates and yields of ciliates and heterotrophic dinoflagellates. J Plankton Res 20:571-584
Tackx MLM, Bakker C, Francke JW, Vink M (1989) Size and phytoplankton selection by Oosterschelde zooplankton. Neth J Sea Res 23:35-43

Tackx MLM, Bakker C, Vanrijswijk P (1990) Zooplankton grazing pressure in the Oosterschelde (the Netherlands). Neth J Sea Res 25:405-415

Tang KW, Taal M (2005) Trophic modification of food quality by heterotrophic protists: species-specific effects on copepod egg production and egg hatching. J Exp Mar Biol Ecol 318:85-98

Teixeira IG, Figueiras FG (2009) Feeding behaviour and non-linear responses in dilution experiments in a coastal upwelling system. Aquat Microb Ecol 55:53-63

Throndsen J (1978) Preservation and storage. In: Sournia A (ed) Phytoplankton manual. UNESCO, Paris, pp 69-74

Tillmann U (2004) Interactions between planktonic microalgae and protozoan grazers. J Eukaryot Microbiol 51:156-168

Tiselius P (1989) Contribution of aloricate ciliates to the diet of Acartia clausi and Centropages hamatus in coastal waters. Mar Ecol Prog Ser 56:49-56

Tomas CR (1996) Identifying marine diatoms and dinoflagellates. Academic Press Inc., San Diego

Tyler JE (1968) The secchi disc. Limnol Oceanogr 13:1-6

Utermöhl H (1958) Zur Vervollkommnung der quantitativen Plankton-Methodik. Mitt Int Ver theor angew Limnol 9:1-38

Vanderploeg H, Scavia D (1979a) Caculation and use of selectivity coefficients of feeding: zooplankton grazing. Ecol Model 7:135-149

Vanderploeg H, Scavia D (1979b) Two electivity indices for feeding with special reference to zooplankton grazing. J Fish Res Board Can 36:362-365

Verity PG (1991) Feeding in planktonic protozoans-evidence for nonrandom acquisition of prey. J Protozool 38:69-76

Wiltshire KH, Dürselen CD (2004) Revision and quality analyses of the Helgoland Reede long-term phytoplankton data archive. Helgol Mar Res 58(4):252-268

Wiltshire KH, Malzahn AM, Wirtz K, Greve W, Janisch S, Mangelsdorf P, Manly BFJ, Boersma M (2008) Resilience of North Sea phytoplankton spring bloom dynamics: an analysis of long-term data at Helgoland Roads. Limnol Oceanogr 53:1294-1302

Xu DP, Song WB, Hu XZ (2005) Morphology of Cyclotrichium taniguchii sp. nov and C. cyclokaryon with establishment of a new genus, Dicyclotrichium gen. nov (Ciliophora: Haptorida). J Mar Biol Assoc UK 85:787-794

Zöllner E, Hoppe HG, Sommer U, Jürgens K (2009) Effect of zooplankton-mediated trophic cascades on marine microbial food web components (bacteria, nanoflagellates, ciliates). Limnol Oceanogr 54:262-275 